\title{
EFFECTS OF IRRADIATION
}

ON

HYDRIDED ZIRCONIUM-URANIUM ALLOY

NAA 120-4 EXPERIMENT

(Title Unclassified)
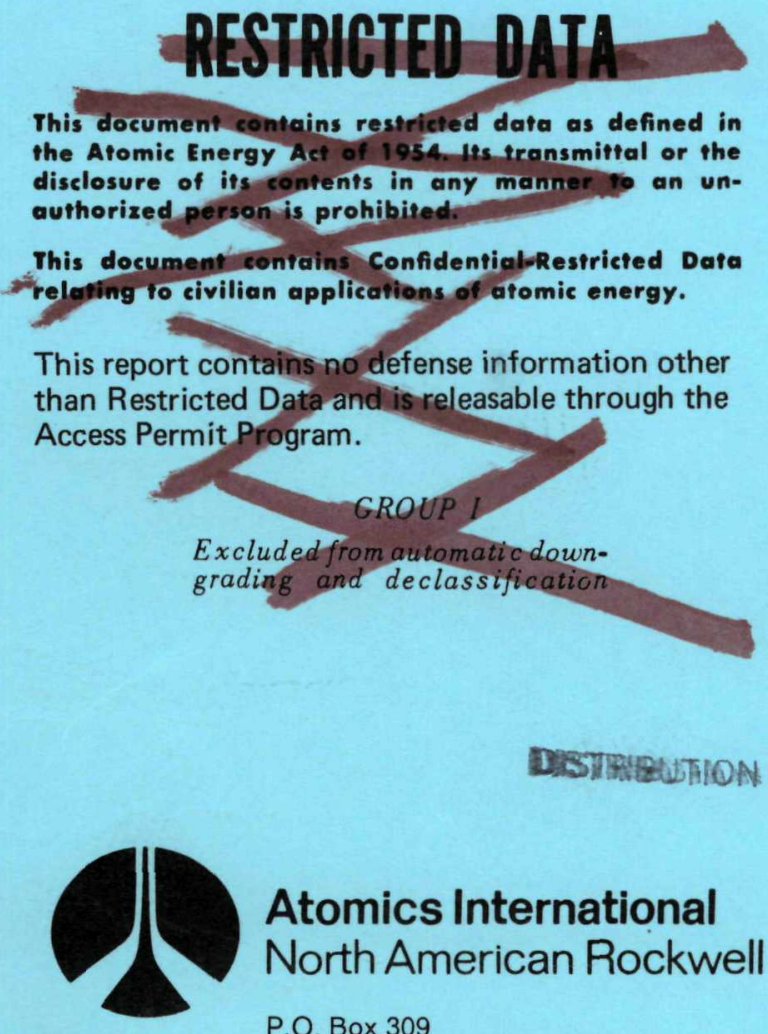

\section{Atomics International}

North American Rockwell

P.O. Box 309

Canoga Park, California 91304 


\section{DISCLAIMER}

This report was prepared as an account of work sponsored by an agency of the United States Government. Neither the United States Government nor any agency Thereof, nor any of their employees, makes any warranty, express or implied, or assumes any legal liability or responsibility for the accuracy, completeness, or usefulness of any information, apparatus, product, or process disclosed, or represents that its use would not infringe privately owned rights. Reference herein to any specific commercial product, process, or service by trade name, trademark, manufacturer, or otherwise does not necessarily constitute or imply its endorsement, recommendation, or favoring by the United States Government or any agency thereof. The views and opinions of authors expressed herein do not necessarily state or reflect those of the United States Government or any agency thereof. 


\section{DISCLAIMER}

Portions of this document may be illegible in electronic image products. Images are produced from the best available original document. 


\section{LEGAL NOTICE}

This report was prepared as an account of work sponsored by the United States Government. Neither the United States nor the United States Atomic Energy Commission, nor any of their employees, nor any of their contractors, subcontractors, or their employees, makes any warranty, express or implied, or assumes any legal liability or responsibility for the accuracy, completeness or usefulness of any information, apparatus, product or process disclosed, or represents that its use would not infringe privately owned rights.

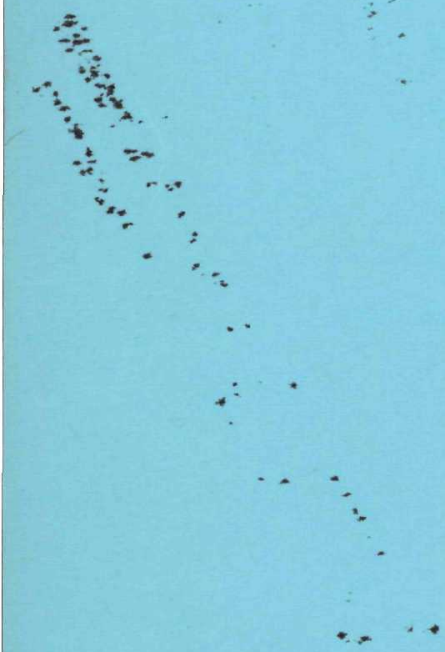




\section{EFFECTS OF IRRADIATION \\ ON}

HYDRIDED ZIRCONIUM-URANIUM ALLOY

NAA 120-4 EXPERIMENT

(Title Unclassified)

NOTICE

By:

N. F. DAVIES

R. E. FORRESTER prepared as an account of work This report was prepared as an Government. Neither sponsored by the United States Gover States Energy the United States nor the Unistration, nor any of Revelopment Administ their contractors, Research andoes, nor any of their contractors, their employees, or their employees, makes any subcontractors, or ther warranty, express or imsibility for the accuracy, completeness lability or responsibility for the accuparatus, product or or usefulness of any information, appat its use would not process disclosed, or represents process disclosed, owned rights.

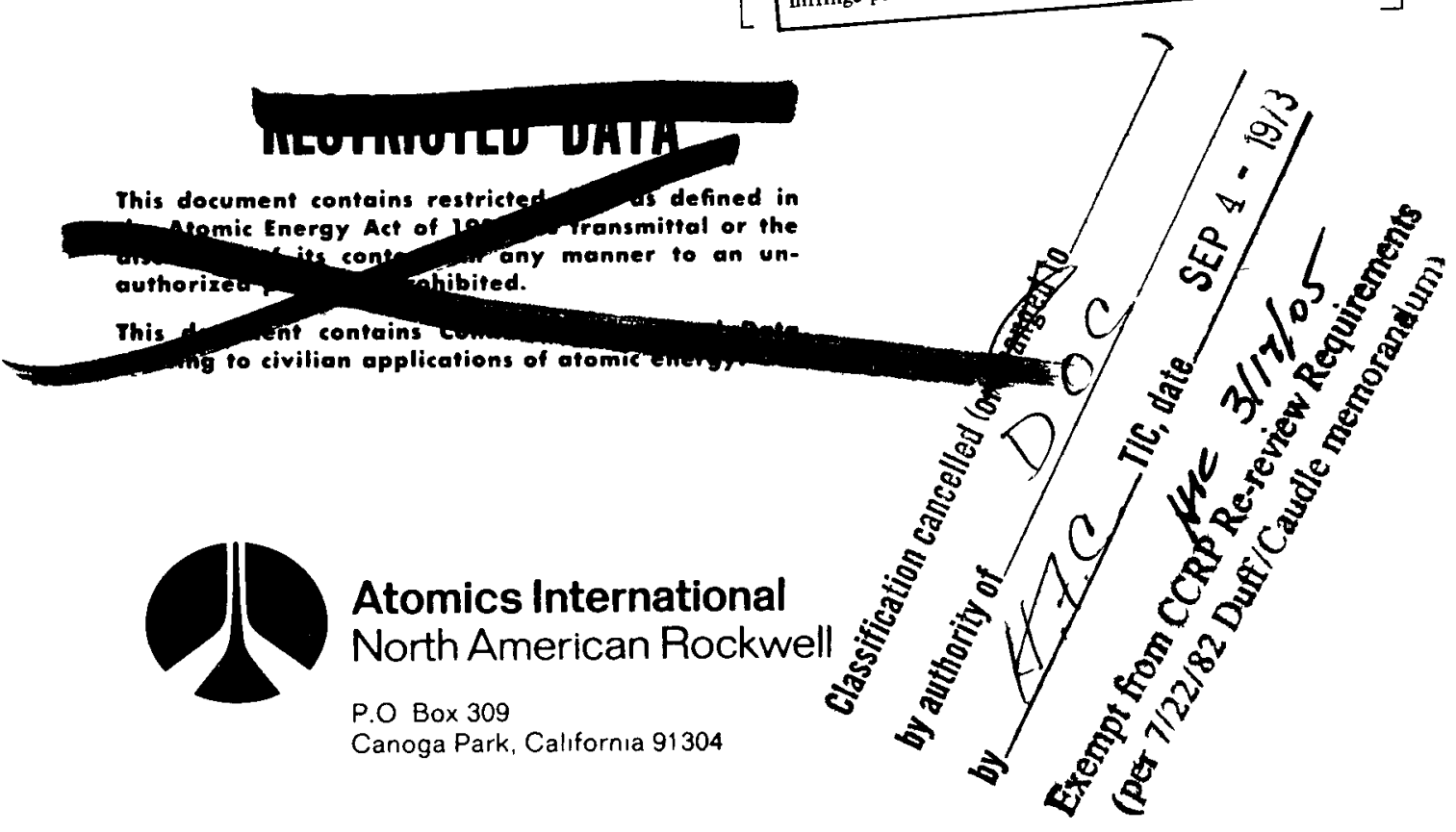




\section{$q^{b}$ \\ SYSTEMS FOR NUCLEAR AUXILIARY POWER (SNAP) REACTOR - SNAP PROGRAM M-3679 (63d Ed.)}

No. of

Copies

AEC Albuquerque Operations Office

A EC Calıforna Patent Group

A EC Chicago Patent Group

A EC Library, Washington

AEC New York Operations Office

AEC Patent Office

AEC SNAP Project Office

Aerojet-General Corporation (NASA)

Aerojet-General Corporation,

Sacramento (AEC)

Aerospace Corporation, Los Angeles

$(A F)$

Air Force Air University Library

$A_{1} r$ Force Headquarters

A 1 Force Strategic Air Command

Air Force Technical Applications

Center

Air Force Weapons Laboratory

Argonne National Laboratory (AEC)

Army Engineer Reactors Group

Army White Sands Missile Range

$A R O$, Inc. (AF)

Battelle Memorial Instıtute (AEC)

Battelle-Northwest (AEC)

Bellcomm, Incorporated (NASA)

Bendix Corporation (NASA)

Brookhaven National Laboratory*(AEC)

Central Intelligence Agency

Donald W. Douglas Laboratories (AEC)

DuPont Company. Alken (AEC)

DuPont Company, Wilmington (AEC)

General Dynamics/Fort Worth (AF)

General Electric Company (AEG) (NASA)

General Electric Company, Cincinnatı (AEC)

General Electric Company, MSD (AFC)

General Electric Company, San Jose (AEC)

Gulf General Atomic Incorporated (AEC)

Institute for Defense Analyses (ARPA)

Jet Propulsion Laboratory (NASA)

Lawrence Radiation Laboratory,

Livermore (AEC)
No. of

Copies

Lockheed-Georgua Company (NASA)

Los Alamos Scientific Laboratory (AEC)

Minnesota Mining and Manufacturing

Company (AEC)

Mound Laboratory (AEC)

NASA Goddard Space Flight Center

NASA Langley Research Center

NASA Lewis Research Center

NASA Marshall Space Flight Center

NASA, Washington

National Reactor Testing Station (INC) (AEC)

Navy Facilities Engineering Command

Navy Office of the Chief of Naval

Operations

Navy Ordnance Laboratory

Navy Ordnance Systems Command

Navy Research Laboratory

Navy Ship Research \& Development Lab.

Navy Ship Systems Command

Headquarters

Navy Space Systems Activity

Oak Ridge National Laboratory (AEC)

Radio Cprporation of America (AEC)

Rand Utsporation (AF)

Sandia Corporation, Albuquerque (AEC)

Sandia Corporation, ARPIC (AEC)

Weiner Associates, Inc (Navy)

Westinghouse Electric Corporation, Lima (NASA)

Westinghouse Electric Corporation. WAL (AEC)

A EC Division of Technical Information Extension

AI Engineering Data (Includes 2 copies to AEC-SNAP Project Office,

2 copies to SAN, 2 copies to C. E.

Johnson, l copy to W. L. Kitterman.

1 copy to Joseph Kenney (WAL).

1 copy to George $P$. Dix, and

1 copy to AEC-WASH 


\section{CONTENTS}

Page

Abstract .............................. 6

I. Introduction and Background ................. 7

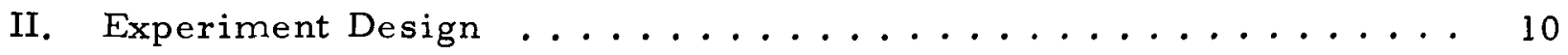

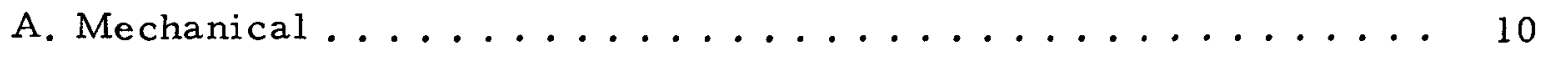

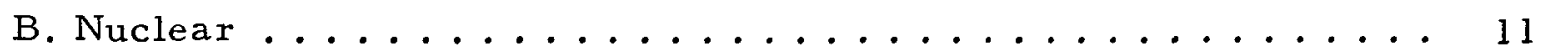

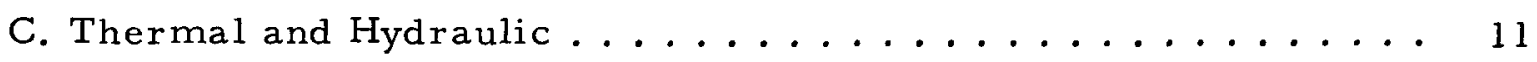

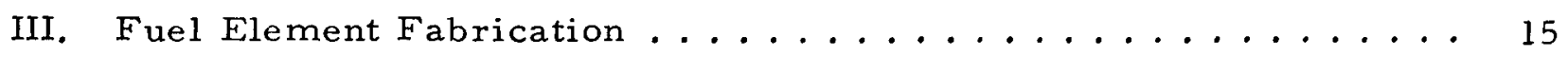

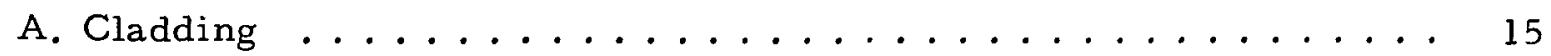

B. Hydrogen Barrier Coating ................... 15

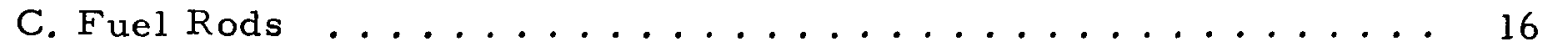

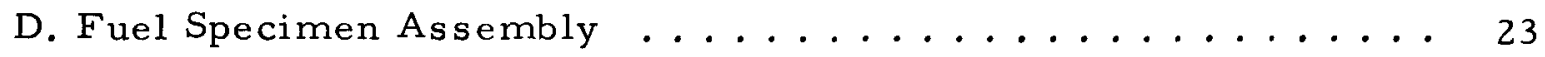

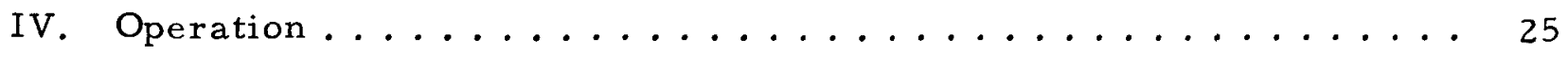

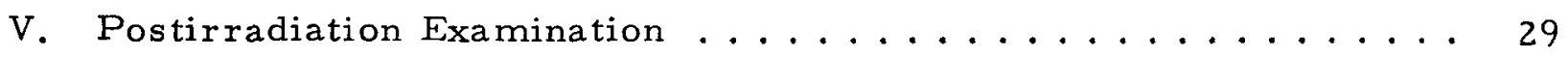

A. Experiment Disassembly and Examination ........... 29

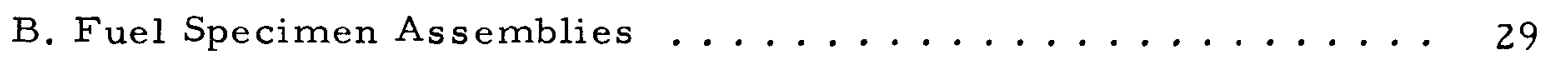

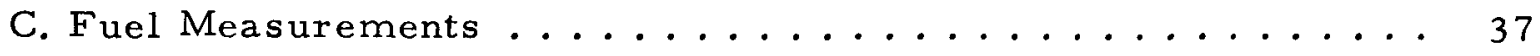

1. Dimensional Measurements ............... 37

2. Fuel Density Measurements ............... 37

3. Fuel Sampling ..................... . . 41

4. Fuel Burnup Measurements ................. 41

5. Hydrogen Measurements .................. 43

D. Fuel Metallographic Examination ................ 43

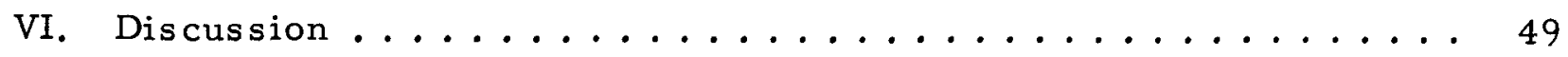

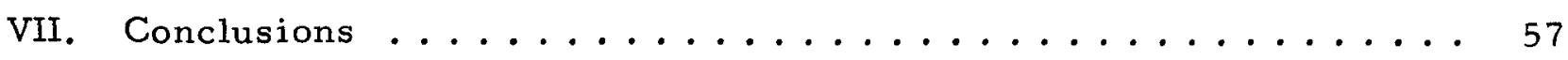

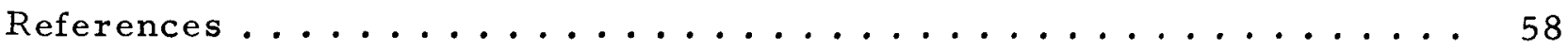




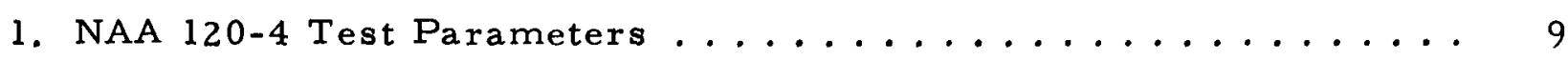

2. Composition of SCB-1 Ceramic Hydrogen Barrier . . . . . . . . 16

3. Chemical Composition of Zirconium-Uranium Hydride Fuel Specimens (Center Fuel Sleeves) ................ 17

4. Chemical Compositions of Zirconium-Uranium Hydride Support Rods and Intermediate and End Fuel Sleeves ......... 18

5. As-Built Dimensions of Test Specimen Support Rods and

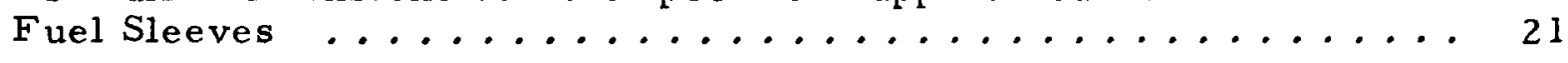

6. Fuel Specimen Permeation Rates and Scanning Test Results . . . . 24

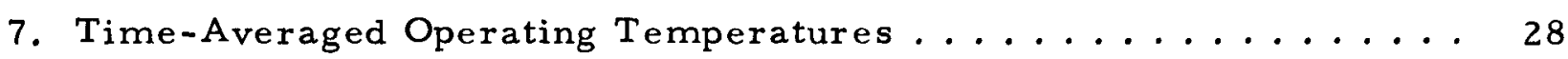

8. NAA 120-4 Fuel Specimen Cladding Dimensional

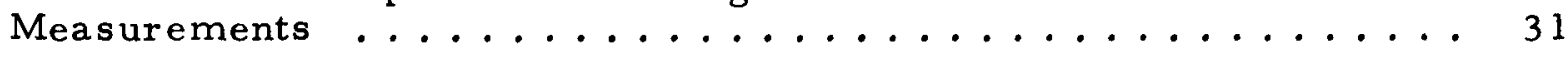

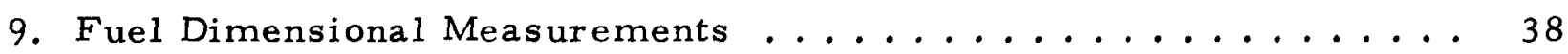

10. Hydrogen-Corrected Fuel Density Measurements .......... 39

11. NAA 120-4 Burnup Analysis ..................... 40

12. NAA 120-4 Hydrogen Analysis ................... . . . . 42

13. Filar Eye Diameter Fuel Measurements vs Pre-Irradiation Fuel Measurements ........................ 44

14. NAA 120-4 Fuel Microstructure Evaluation (Metallographic

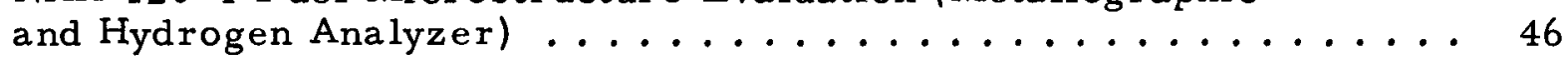

15. Summary of NAA 120-4 Experiment Data . . . . . . . . . . 54

\section{FIGURES}

1. Zirconium-Hydrogen Phase Diagram ............... 8

2. NAA 120-4 Flux Monitor Positions .................. 12

3. Fuel Specimen Configuration

a. Typical Noninstrumented Fuel Specimen . . . . . . . . . . 14

b. Typical Instrumented Fuel Specimen . . . . . . . . . . . . 14

4. Typical NAA 120-4 Fuel Specimen Assembly . . . . . . . . . . 20

5. Power-Normalized Fuel-to-Cladding $\Delta \mathrm{T}$ vs Time

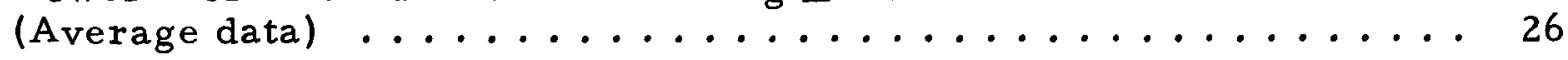

6. Neutron Radiographs of the NAA 120-4 Experiment ........ 30 


\section{FIGURES}

7. Specimen E-1

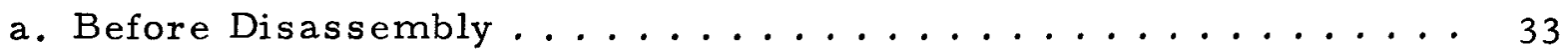

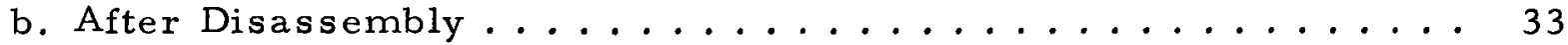

c. Inside Fuel Support . . . . . . . . . . . . . . . 33

d. Typical Area of Center Fuel Sleeve .............. 33

8. Specimen $M-1$

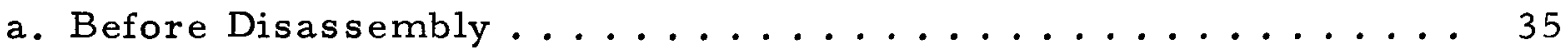

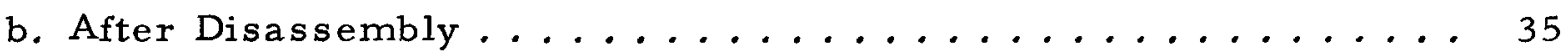

9. Specimen E-4

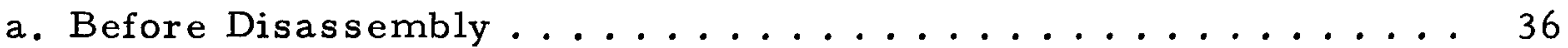

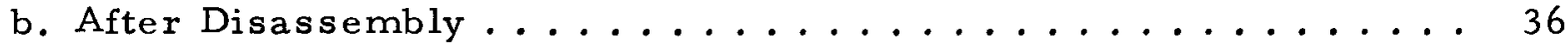

10. Typical Photomicrographs of the NAA 120-4 Fuel

a. E-3 Fuel Sleeve $(\delta+\epsilon) \ldots \ldots \ldots 47$

b. H-5 Fuel Sleeve $(\delta) \ldots \ldots \ldots \ldots$

c. V-1 Fuel Sleeve $\left(\delta+\epsilon^{\prime}\right) \ldots \ldots \ldots \ldots \ldots$

11. Radial Fuel Growth vs Time (Based on fuel-cladding $\Delta \mathrm{T}$ ) . . . . . 48

12. Volume Increase vs Burnup for the $1300^{\circ} \mathrm{F}$ Subcapsules (Based on fuel-cladding $\Delta \mathrm{T}) \ldots \ldots \ldots \ldots$

13. Volume Increase vs Burnup for the $1500^{\circ} \mathrm{F}$ Subcapsules (Based on fuel-cladding $\Delta \mathrm{T}$ ) $\ldots \ldots \ldots \ldots$

14. Volume Increase vs Burnup for the $1600^{\circ} \mathrm{F}$ Subcapsules (Based on fuel-cladding $\Delta \mathrm{T}) \ldots \ldots \ldots$

15. Fuel Volume Changes vs Fuel Diameter Changes (Corrected for hydrogen loss) ...................... 52

16. Burnup-Corrected Volume Change vs Reciprocal Calculated

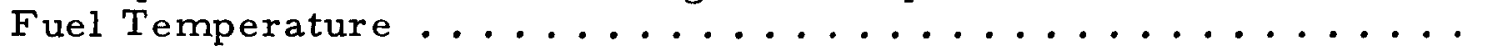




\section{ABSTRACT}

In the NAA 120-4 Experiment, 17 fuel specimens were irradiated simultaneously in a single facility at the Hanford K-East Reactor, to a target burnup of $\sim 0.9$ metal at. $\%$. The experiment was statistically designed to evaluate the performance of SNAP fuel materials as a function of fuel composition and temperature. The specimens were contained in five separate experimental units, which provided a means of controlling the maximum fuel temperatures at different levels, ranging from 1300 to $1600^{\circ} \mathrm{F}$. Design compositions included 1.70 and $1.85 \mathrm{H} / \mathrm{Zr}$ ratios. The maximum temperature of the Hastelloy $X$ cladding and the hydrogen barrier (SCB-1 ceramic) did not exceed $1300^{\circ} \mathrm{F}$ for any extended periods.

Burnup analysis showed that the NAA 120-4 Experiment achieved a burnup within $10 \%$ of its target requirements.

Postirradiation examination showed that the cladding was intact on all 17 fuel specimens. Disassembly of the fuel specimens showed cracking had occurred in 8 of the 17 central fuel sleeves (test fuel specimens). Besides exhibiting a strong temperature dependence for fuel swelling, this experiment also confirmed the existence of the postulated "offset" fuel growth which saturates early in life.

This report describes the fuel element fabrication, experiment design, and hot laboratory examination. It does not include that portion of the irradiation that would disclose information on Hanford production. 


\section{INTRODUCTION AND BACKGROUND}

The SNAP* 8 fuel element concept is based on the use of a homogenous fuelmoderator system. A dispersion of metallic uranium in a zirconium hydride matrix results from hydriding $\mathrm{Zr}-10 \mathrm{wt} \% \mathrm{U}$ to $\mathrm{N}_{\mathrm{H}}=6.016 .0 \times 10^{22}$ atoms $\mathrm{H} / \mathrm{cc}$ fuel). Carbon is added to the alloy as a grain refiner. This alloy constitutes the fuel-moderator combination in the compact SNAP reactors. The fuel-moderator rods are clad in Hastelloy $N$, which has a continuous layer of ceramic on its inner surface to inhibit hydrogen permeation through the cladding at reactor operating temperatures.

Axial and radial temperature profiles exist across the fuel element in an operating SNAP reactor. The axial profile results in operation of the highertemperature zone of the element, near the reactor outlet, in the face-centeredcubic $\delta$-hydride phase region. The lower-temperature end of the element, by reason of the temperature gradient, operates in the face-centered-tetragonal $\epsilon$-hydride phase region. The radial gradient superimposes a segregation of $\delta$ phase along the centerline, and $\epsilon$ to the surface in the boundary region. The compositional region of interest for a SNAP 8 reactor is presented in the zirconium-hydrogen phase diagram, Figure 1. Excessive loss of hydrogen, and entry into the $\beta+\delta$ phase regions, is considered an undesirable condition. $(1,2)$

At the initiation of the SNAP 8 Fuel Element Irradiation Program, dimensional stability of the fuel-moderator was considered of key importance, based on results of a series of earlier irradiation tests. ${ }^{(3,4)}$

Part of the program involved full-scale and sublength prototype irradiations, to determine the performance capabilities of the fuel element configuration as a function of temperatures, burnup, burnup rate, and fuel material composition and structure. The three sublength prototype series $(3,5,6)$ have been completed. The initial full-scale experiment, NAA $82-1^{(7)}$ (actually a SNAP 2 experiment, but with applicable results), has been completed; the NAA 121 series of fullscale fuel elements has been irradiated, and is undergoing postirradiation examination.

*SNAP designates a System for Nuclear Auxiliary Power. The SNAP 8 reactor is rated at $600 \mathrm{kwt}$ for $10,000 \mathrm{hr}$. 


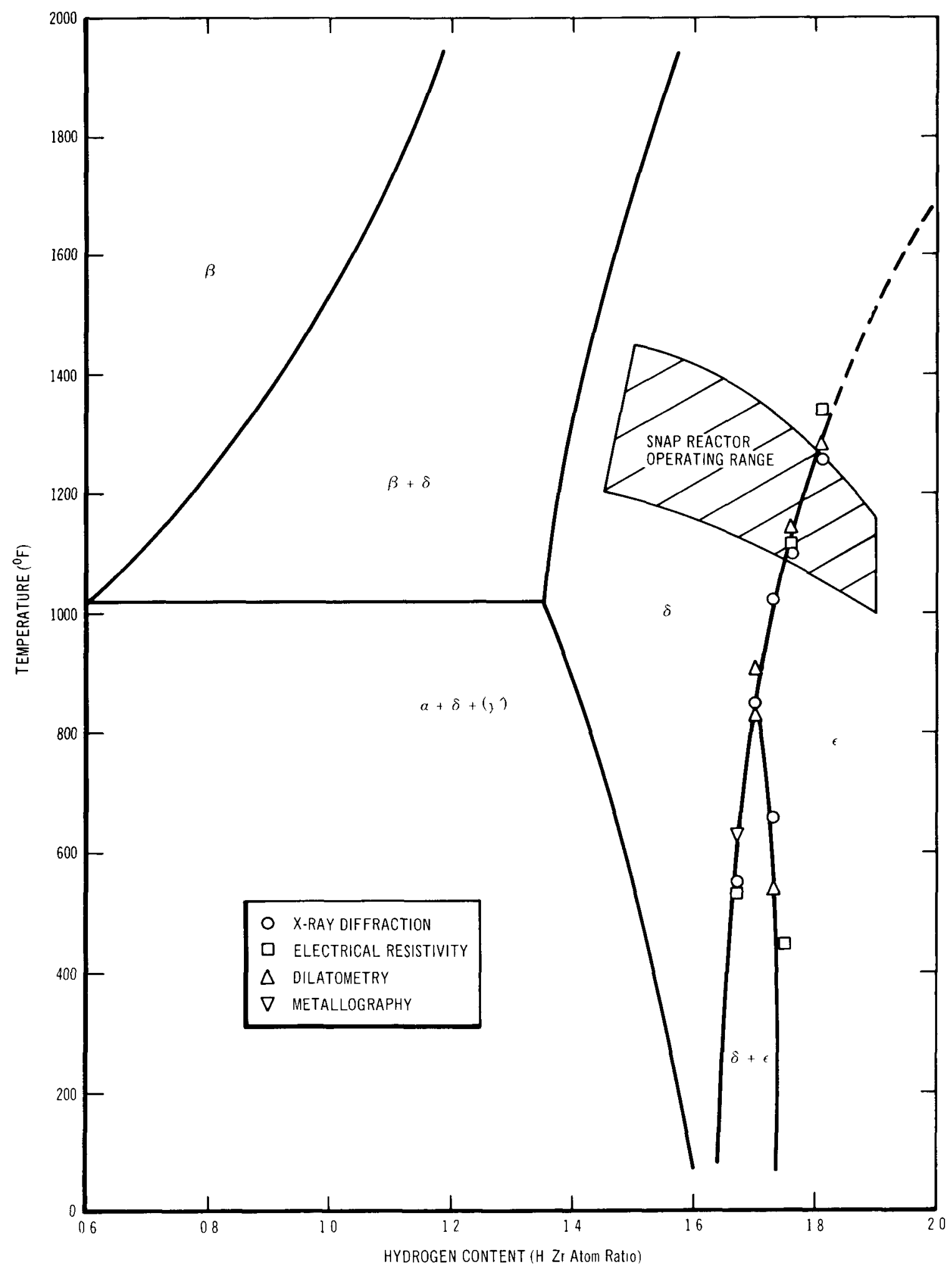

$118-68 \mathrm{UNCL}$

Figure 1. Zirconium-Hydrogen Phase Diagram

(Figure Unclassified)

$$
\begin{gathered}
\mathrm{AI}-\mathrm{AEC}-12963 \\
8
\end{gathered}
$$


The basic objective of the NAA 120-4, reported here, was to obtain a better understanding of the behavior of hydrided uranium-zirconium alloy fuel during irradiation. Unlike the sublength and full-scale fuel element tests, the NAA120-4 was designed to operate at constant fuel temperatures $(1300,1500$, and $1600^{\circ} \mathrm{F}$ ), rather than at constant cladding temperatures. Specimens having $\mathrm{H} / \mathrm{Zr}$ ratios of 1.70 and 1.85 were tested at each temperature. Target burnup for the experiment was 0.9 metal at. \%. Five of the 17 fuel specimens which were irradiated contained in-fuel thermocouples. These thermocouples were included for temperature control and for measurement of fuel-to-cladding $\Delta \mathrm{T}$, from which calculations of fuel swelling were made. The specimen test parameters are shown in Table 1.

TABLE 1

NAA 120-4 TEST PARAMETERS

\begin{tabular}{|c|c|c|}
\hline $\begin{array}{c}\text { Fuel } \\
\text { Specimens }\end{array}$ & $\mathrm{H} / \mathrm{Zr}$ & $\begin{array}{c}\text { Irradiation } \\
\text { Temperature } \\
\left({ }^{\circ} \mathrm{F}\right)\end{array}$ \\
\hline$E-1^{* *}$ & 1.85 & 1300 \\
\hline$E-2$ & 1.70 & 1300 \\
\hline E-3 & 1.70 & 1300 \\
\hline E-4 & 1.85 & 1300 \\
\hline$E-5$ & 1.85 & 1300 \\
\hline $\mathrm{H}-1^{*}$ & 1.70 & 1500 \\
\hline $\mathrm{H}-2$ & 1.70 & 1500 \\
\hline $\mathrm{H}-3$ & 1.70 & 1500 \\
\hline $\mathrm{H}-4$ & 1.85 & 1500 \\
\hline $\mathrm{H}-5$ & 1.85 & 1500 \\
\hline$M-1^{*}$ & 1.70 & 1600 \\
\hline$M-2$ & 1.70 & 1600 \\
\hline$M-3$ & 1.70 & 1600 \\
\hline$M-4$ & 1.85 & 1600 \\
\hline$M-5$ & 1.85 & 1600 \\
\hline$V-1 *$ & 1.70 & 1500 \\
\hline $\mathrm{V}-2^{* *}$ & 1.70 & 1500 \\
\hline
\end{tabular}

*Specimens equipped with in-fuel thermocouples and hydrogen pressure sensors. 


\section{EXPERIMENT DESIGN}

\section{A. MECHANICAL}

The NAA 120-4 Experiment consisted of five separate subcapsules, designed to operate at three different fuel centerline temperatures. Three of the subcapsules contained five fuel specimen assemblies each, and the other two subcapsules [the phase change experiments ${ }^{(8)}$ ] each contained one fuel specimen assembly. Each fuel specimen assembly was instrumented with three cladding thermocouples. In addition, one specimen in each subcapsule was instrumented with one in-fuel thermocouple and a hydrogen pressure probe.

The phase change fuel specimen assemblies were designed so that in-fuel temperature and fuel dissociation pressure could be continuously monitored, and hydrogen could be added or removed from the fuel during operation.

The fuel specimen as semblies were centered in $0.710-$ in. ID stainless steel fuel sleeves. The entire void space surrounding the fuel specimen assemblies was filled with $\mathrm{NaK}-78$ containing a maximum of 14 ppm oxygen. A bellows was used to compensate for $\mathrm{NaK}$ expansion and contraction. The bellows was contained in a bellows compartment which was designed so that, even if the bellows failed, each fuel specimen assembly would still be submerged in NaK.

The NaK chamber of each capsule was surrounded by a precisely machined gas annulus. The temperatures of the fuel specimens were controlled by varying the mixture of helium and argon in this annulus. The gas annulus, formed by the OD of the $\mathrm{NaK}$ compartment and the ID of the outer shell, was essentially the same for all of the subcapsules $-0.008 \mathrm{in}$. in width. The outer shell of each subcapsule was a 1.0-in. OD, 0.065-in. wall, Type 304 stainless steel tube. Each of the five subcapsules had a separate gas temperature control system. Fuel centerline temperature of the instrumented specimen was used for control of each capsule.

The experiment was instrumented with 62 thermocouples, 5 pressure sensors, and 4 flux monitors. A total of 51 Chromel-Alumel thermocouples were banded to the fuel specimen cladding tubes. The remainder of the thermocouples were Chromel-Alumel, and were used to monitor reactor coolant temperature and peripheral experiment components. The flux monitors used in the experiment were Reuter-Stokes, Type RSN-202, Neutron Detectors, and were located 
as shown in Figure 2. The pressures associated with E-1, H-1, and M-1 specimens were monitored by null-balance sensors located near the specimen. The pressures of the $\mathrm{V}-1$ and $\mathrm{V}-2$ specimens were monitored, using direct communication lines and ex-reactor strain gage transducers. A neutron dosimeter was banded to each fuel specimen. The dosimeters consisted of $\mathrm{Fe}, \mathrm{Ni}$, and Co wires in a stainless steel tube with both ends brazed closed.

\section{B. NUCLEAR}

The 2A Test Facility in the HAPO KE Reactor was chosen for the NAA 120-4 Experiment, because it fulfilled the requirements of a high thermal neutron flux that was reasonably flat over a distance that exceeded the length of the experiment. A shroud tube was required, in order to obtain the specified heat generation rate in the fuel during irradiation. The shroud tube design criteria were determined from: (1) lattice cell calculations that provided relative spatial thermal neutron flux distributions, and (2) the measured unperturbed thermal neutron flux in the test facility. (9) The cell calculations were performed, ${ }^{(10)}$ employing a 30 thermal group transport theory code ${ }^{(11)}$ using cylindrical geometry.

\section{THERMAL AND HYDRAULIC}

Fuel temperatures in the experiment were controlled by varying the amounts of helium and argon in the gas annuli (helium has good thermal conduction, argon poor conduction). The design of the temperature control annulus was accomplished ${ }^{(12)}$ by using the one-dimensional heat transfer code, GASP. (13) The nominal gas composition was fixed at $50 \% \mathrm{He}-50 \% \mathrm{~A}$, allowing a good temperature control range.

The axial temperature profile of the fuel specimens would as sume an approximate cosine shape, if the axial heat losses were not compensated for. The temperature profile was markedly improved (made essentially isothermal) by the use of heat barriers (enlarged gas annuli) at both ends of the fuel specimens, and by the use of 20 wt $\%$ fully enriched uranium end sleeves. The optimum location of these barriers was determined by an iterative process, in which the thickness and location of the gas annulus step and the length and thickness of the 20 wt $\%$ end sleeves were varied. The heat barrier optimization analyses ${ }^{(12)}$ were performed in an $\mathbf{r}-\mathrm{Z}$ geometry, using the computer code HEATING. 


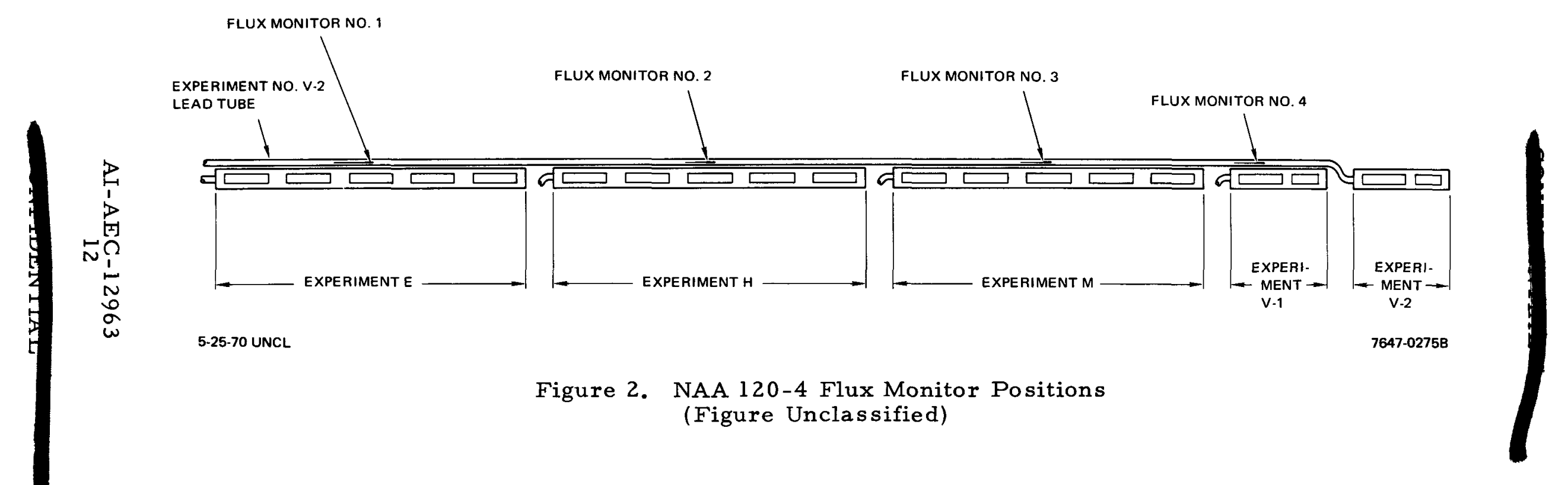


Prior to insertion of the experiment into the KE Reactor, the assembly was flow tested in a hydraulic loop at Hanford. The hydraulic tests consisted of: (1) overall experiment pressure drop, (2) capsule pressure drop, and (3) flowsplit inside and outside the shroud tube. Test results showed an overall experiment pressure drop of 14 psi. The flow split on Capsules 1, 2, and 3 was $30 \%$ inside and $70 \%$ outside of the shroud tube. The corresponding ratios for Capsules 4 and 5 were 39 and $61 \%$. 


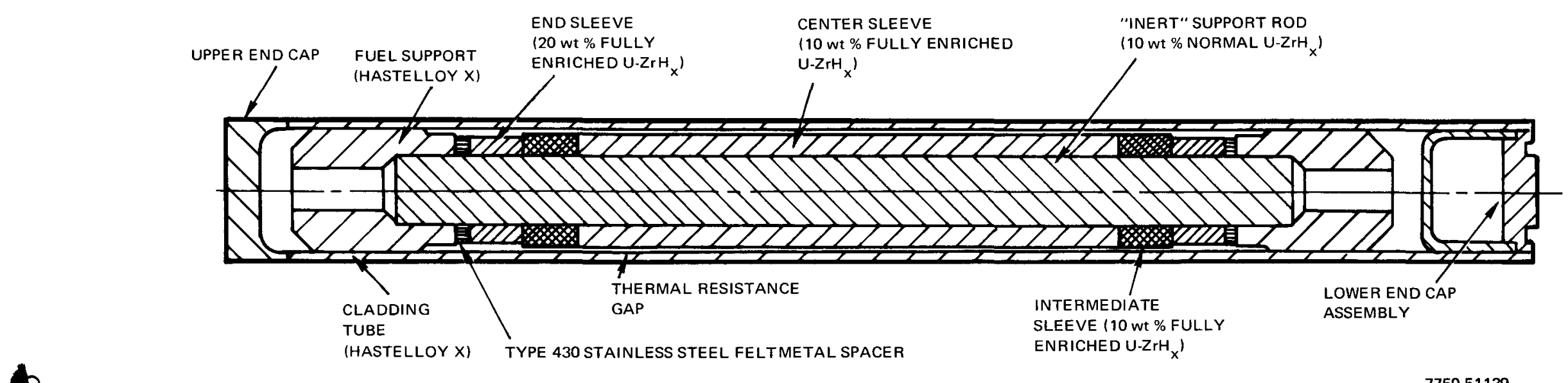

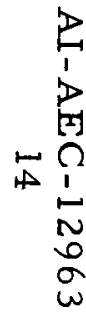

a. Typical Noninstrumented Fuel Specimen

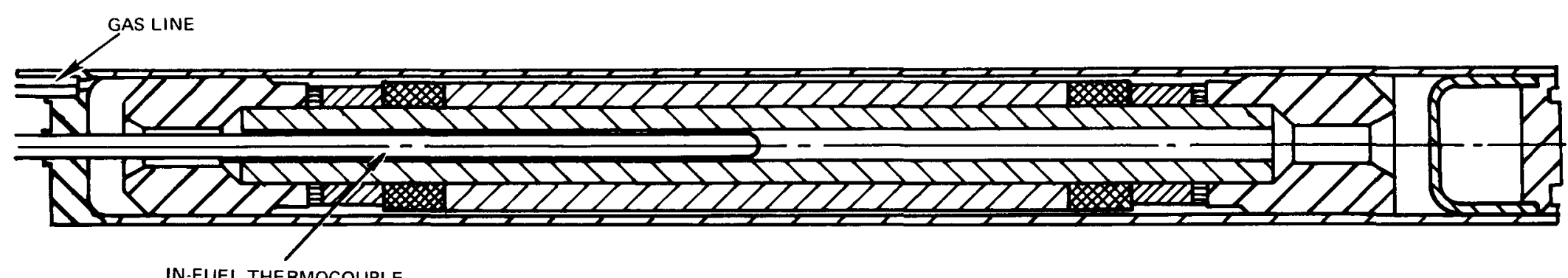

IN-FUEL THERMOCOUPLE

$7759-51140$

b. Typical Instrumented Fuel Specimen

Figure 3. Fuel Specimen Configuration (Figure Unclassified) 


\section{FUEL ELEMENT FABRICATION}

The NAA 120-4 Experiment contained 17 fuel specimen assemblies. Each as sembly consisted of five fuel sleeves containing fully enriched $U-\mathrm{ZrH}_{\mathbf{x}}$ mounted on an "inert" support rod of normal U- $\mathrm{ZrH}_{\mathrm{x}}$, as shown in Figure 3. The end fuel sleeves contained 20 wt $\%$ of $93 \%$ enriched $\mathrm{U}-\mathrm{ZrH}_{\mathbf{x}}$, while the intermediate and center sleeves contained 10 wt $\%$ fully enriched $U-\mathrm{ZrH}_{\mathbf{x}} \cdot$ The end and intermediate sleeves, as well as the support rod, were considered as "throw-away" pieces, merely included to provide the proper conditions for the test specimen (center fuel sleeve).

The specimen assemblies were centered in the cladding by the use of Hastelloy $\mathrm{X}$ fuel supports. Compressible 10\% dense Type 430 stainless steel Feltmetal ${ }^{*}$ was placed between the end fuel sleeves and the fuel supports, to prevent the fuel sleeves from separating. The specimens were then clad in Hastelloy $\mathrm{X}$ which had been coated with a ceramic hydrogen-permeation-barrier material (SCB-1).

\section{A. CLADDING}

The NAA 120-4 fuel specimen as sembly cladding tube was nominally 0.480in. $O D$ by 0.030-in. wall, Hastelloy X-285. The end closures consisted of Hastelloy $\mathrm{X}$ machined from bar stock. The lower end closure consisted of an end cap and inner cup, E. B. ${ }^{\dagger}$ welded together to form a vacuum thermal barrier. The instrumented assemblies contained a Hastelloy $X$ thermocouple well and a Type 304 stainless steel pressure tap, E.B. welded to the upper end cap. The fuel supports were also machined from Hastelloy $X$, and had a thin coating of tungsten vapor-deposited on them, to prevent adherence of the Hastelloy $X$ to the SCB-1 ceramic coating. In a test, prior to assembly of the experiment, the tungsten was found to be compatible with the SCB-1 ceramic coating.

\section{B. HYDROGEN BARRIER COATING}

The cladding tubes, end closures, and the outside of the thermocouple wells were coated with SCB-1 ceramic hydrogen barrier. The composition of the ceramic *Trademark of Huyck Metals, Inc. †Electron Beam 
is given in Table 2. The ceramic was applied as an aqueous slip, air dried, and fired for $5 \mathrm{~min}$ at $2125^{\circ} \mathrm{F}$. The lower end cap assembly, which comprised the final specimen as sembly closure, was coated with SCB-1 ceramic prior to welding into the tube assembly. After the lower end cap assembly had been welded into the assembly, a fusion cycle was performed. The purpose of the fusion cycle was to form a ceramic-to-ceramic bond between the lower end cap assembly and the tube assembly. The fusion bond was formed by induction heating the lower end of the assembly for $1-1 / 2 \mathrm{~min}$ at $1950^{\circ} \mathrm{F}$ in a vacuum. Nominal ceramic layer thickness on the cladding tubes ranged from 1.5 to 2.0 mils. (16)

TABLE 2

COMPOSITION OF SCB-1 CERAMIC HYDROGEN BARRIER

\begin{tabular}{|c|c|}
\hline \multicolumn{2}{|l|}{ Frit } \\
\hline Material & wt $\%$ \\
\hline $\mathrm{SiO}_{2}$ & $45.2 \pm 0.18$ \\
\hline $\mathrm{TiO}_{2}$ & $12.6 \pm 0.05$ \\
\hline $\mathrm{Al}_{2} \mathrm{O}_{3}$ & $7.2 \pm 0.03$ \\
\hline $\mathrm{BaO}_{2}$ & $35.9 \pm 0.14$ \\
\hline \multicolumn{2}{|l|}{ Slip } \\
\hline Material & Weight (gm) \\
\hline SCB-1 (Frit) & $485.0 \pm 1.95$ \\
\hline Ferro Black Label Clay & $15.0 \pm 0.06$ \\
\hline Chrome Oxide $\left(\mathrm{Cr}_{2} \mathrm{O}_{3}\right)$ & $5.0 \pm 0.05$ \\
\hline Bentonite & $1.25 \pm 0.05$ \\
\hline Distilled Water & $350.0 \pm 1.40$ \\
\hline
\end{tabular}

\section{FUEL RODS}

Fuel material was prepared by melting, extruding, and hydriding. The melt stock consisted of reactor grade zirconium sponge, reactor grade uranium, and high-purity zirconium carbide powder. Alloys were prepared by arc-melting under a vacuum of $\sim 1 \mu$.

Extrusion was carried out at $1000^{\circ} \mathrm{F}$, using a reduction in area of $\sim 10$ to 1 . The fuel was then rough machined into the various required configurations. 
Hydriding was accomplished by placing the fuel in an evacuated retort held at $1650^{\circ} \mathrm{F}$, and slowly introducing a known volume of hydrogen gas into the retort. (17) Following hydriding, the sleeves and support rods were machined to the required dimensions. Tables 3 and 4 present compositions of the various finished specimen as sembly parts.

TABLE 3

\section{CHEMICAL COMPOSITION O.F ZIRCONIUM-URANIUM HYDRIDE FUEL SPECIMENS \\ (Center Fuel Sleeves)}

\begin{tabular}{|c|c|c|c|c|c|c|c|}
\hline \multirow{2}{*}{$\begin{array}{l}\text { Fuel } \\
\text { Speci- } \\
\text { men }\end{array}$} & \multirow{2}{*}{$\begin{array}{c}\text { Fuel } \\
\text { Rod } \\
\text { Number }\end{array}$} & \multirow{2}{*}{$\begin{array}{l}\text { Zirco- } \\
\text { nium } \\
\text { (wt } \% \text { ) }\end{array}$} & \multicolumn{2}{|c|}{ Uranium } & \multirow{2}{*}{$\begin{array}{c}\text { Carbon } \\
\text { (wt \%) }\end{array}$} & \multicolumn{2}{|c|}{ Hydrogen } \\
\hline & & & $\begin{array}{l}\text { Total } \\
\text { (wt \%) }\end{array}$ & $\begin{array}{c}\text { Enrichment } \\
(\%)\end{array}$ & & $(w t \%)$ & $\mathrm{H} / \mathrm{Zr}$ \\
\hline E-1 & $707-D-1-B$ & Balance & 10.52 & 93.05 & 0.13 & 1.78 & 1.85 \\
\hline$E-2$ & $692-B-2-B$ & Balance & 10.08 & 93.12 & 0.14 & 1.66 & 1.72 \\
\hline$E-3$ & $692-B-1-A$ & Balance & 10.08 & 93.12 & 0.14 & 1.65 & 1.72 \\
\hline$E-4$ & $692-A-1-B$ & Balance & 10.10 & 93.13 & 0.14 & 1.78 & 1.86 \\
\hline$E-5$ & $707-D-1-C$ & Balance & 10.52 & 93.05 & 0.13 & 1.78 & 1.86 \\
\hline $\mathrm{H}-\mathrm{l}$ & $692-C-1-C$ & Balance & 10.05 & 93.14 & 0.12 & 1.65 & 1.70 \\
\hline $\mathrm{H}-2$ & $707-C-3-C$ & Balance & 10.56 & 93.05 & 0.12 & 1.65 & 1.71 \\
\hline $\mathrm{H}-3$ & $707-D-2-C$ & Balance & 10.52 & 93.05 & 0.13 & 1.63 & 1.70 \\
\hline $\mathrm{H}-4$ & $692-D-1-B$ & Balance & 9.98 & 93.11 & 0.15 & 1.79 & 1.85 \\
\hline $\mathrm{H}-5$ & $692-D-1-C$ & Balance & 9.98 & 93.11 & 0.15 & 1.78 & 1.85 \\
\hline$M-1$ & $707-D-2-B$ & Balance & 10.52 & 93.05 & 0.13 & 1.64 & 1.71 \\
\hline$M-2$ & $692-B-2-A$ & Balance & 10.08 & 93.12 & 0.14 & 1.65 & 1.72 \\
\hline$M-3$ & $692-C-2-A$ & Balance & 10.05 & 93.14 & 0.12 & 1.64 & 1.70 \\
\hline$M-4$ & $692-C-3-A$ & Balance & 10.05 & 93.14 & 0.12 & 1.78 & 1.85 \\
\hline$M-5$ & $692-D-1-A$ & Balance & 9.98 & 93.11 & 0.15 & 1.77 & 1.84 \\
\hline$V-1$ & $707-D-2-A$ & Balance & 10.52 & 93.05 & 0.13 & 1.64 & 1.71 \\
\hline$V-2$ & $692-B-2-C$ & Balance & 10.08 & 93.12 & 0.14 & 1.65 & 1.71 \\
\hline
\end{tabular}

Dimensional measurements, taken on the support rods and fuel sleeves prior to assembly, are listed in Table 5. Figure 4 shows a typical fuel assembly, prior to insertion into the cladding tube assembly. 
TABLE 4

CHEMICAL COMPOSITIONS OF ZIRCONIUM-URANIUM HYDRIDE SUPPORT RODS AND INTERMEDIATE AND END FUEL SLEEVES

\begin{tabular}{|c|c|c|c|c|c|c|c|}
\hline \multirow{2}{*}{ Description } & \multirow{2}{*}{$\begin{array}{l}\text { Fuel } \\
\text { Rod } \\
\text { Number }\end{array}$} & \multirow{2}{*}{$\begin{array}{l}\text { Zirco- } \\
\text { nium } \\
(\text { wt } \%)\end{array}$} & \multicolumn{2}{|c|}{ Uranium } & \multirow{2}{*}{$\begin{array}{r}\text { Carbon } \\
(\text { wt } \%)\end{array}$} & \multicolumn{2}{|c|}{ Hydrogen } \\
\hline & & & $\begin{array}{r}\text { Total } \\
(\text { wt } \%)\end{array}$ & $\begin{array}{c}\text { Enrichment } \\
(\%)\end{array}$ & & (wt \%) & $\mathrm{H} / \mathrm{Zr}$ \\
\hline $\begin{array}{l}\text { E-1 } \\
\text { Support Rod } \\
\text { Intermediate }\end{array}$ & $392-A-2-A$ & Balance & 10.62 & Normal & 0.12 & 1.78 & 1.85 \\
\hline $\begin{array}{l}\text { Sleeve } \\
\text { End Sleeve }\end{array}$ & $\begin{array}{l}707-D-1-B \\
630-A-5\end{array}$ & $\begin{array}{l}\text { Balance } \\
\text { Balance }\end{array}$ & $\begin{array}{l}10.52 \\
18.77\end{array}$ & $\begin{array}{l}93.05 \\
92.5\end{array}$ & $\begin{array}{l}0.13 \\
0.09\end{array}$ & $\begin{array}{l}1.78 \\
1.62\end{array}$ & $\begin{array}{l}1.85 \\
1.85\end{array}$ \\
\hline $\mathrm{E}-2$ & & & & & & & \\
\hline $\begin{array}{l}\text { Support Rod } \\
\text { Intermediate }\end{array}$ & $391-C-3-B$ & Balance & 10.64 & Normal & 0.17 & 1.62 & 1.69 \\
\hline $\begin{array}{l}\text { Sleeve } \\
\text { End Sleeve }\end{array}$ & $\begin{array}{l}692-B-2-B \\
630-A-7-A\end{array}$ & $\begin{array}{l}\text { Balance } \\
\text { Balance }\end{array}$ & $\begin{array}{l}10.08 \\
18.50\end{array}$ & $\begin{array}{l}93.12 \\
92.5\end{array}$ & $\begin{array}{l}0.14 \\
0.11\end{array}$ & $\begin{array}{l}1.66 \\
1.49\end{array}$ & $\begin{array}{l}1.72 \\
1.70\end{array}$ \\
\hline $\begin{array}{l}\text { E-3 } \\
\text { Support Rod }\end{array}$ & $391-A-3-C$ & Balance & 10.63 & Normal & 0.16 & 1.64 & 1.71 \\
\hline $\begin{array}{l}\text { Intermediate } \\
\text { Sleeve } \\
\text { End Sleeve }\end{array}$ & $\begin{array}{l}692-B-1-A \\
630-A-3-B\end{array}$ & $\begin{array}{l}\text { Balance } \\
\text { Balance }\end{array}$ & $\begin{array}{l}10.08 \\
18.30\end{array}$ & $\begin{array}{l}93.12 \\
92.5\end{array}$ & $\begin{array}{l}0.14 \\
0.11\end{array}$ & $\begin{array}{l}1.65 \\
1.50\end{array}$ & $\begin{array}{l}1.72 \\
1.71\end{array}$ \\
\hline $\begin{array}{l}\text { E-4 } \\
\text { Support Rod } \\
\text { Intermediate }\end{array}$ & $391-D-2-B$ & Balance & 10.64 & Normal & 0.17 & 1.76 & 1.85 \\
\hline $\begin{array}{c}\text { Sleeve } \\
\text { End Sleeve }\end{array}$ & $\begin{array}{l}692-A-1-B \\
630-A-8\end{array}$ & $\begin{array}{l}\text { Balance } \\
\text { Balance }\end{array}$ & $\begin{array}{l}10.10 \\
18.55\end{array}$ & $\begin{array}{l}93.13 \\
92.5\end{array}$ & $\begin{array}{l}0.14 \\
0.11\end{array}$ & $\begin{array}{l}1.78 \\
1.49\end{array}$ & $\begin{array}{l}1.86 \\
1.70\end{array}$ \\
\hline$E-5$ & & & & & & & \\
\hline $\begin{array}{l}\text { Support Rod } \\
\text { Intermediate }\end{array}$ & $392-A-3-B$ & Balance & 10.62 & Normal & 0.14 & 1.78 & 1.85 \\
\hline Sleeve & $707-D-1-C$ & Balance & 10.52 & 93.05 & 0.13 & 1.78 & 1.86 \\
\hline End Sleeve & $630-A-8$ & Balance & 18.55 & 92.5 & 0.11 & 1.49 & 1.70 \\
\hline $\mathrm{H}-1$ & & & & & & & \\
\hline $\begin{array}{l}\text { Support Rod } \\
\text { Intermediate }\end{array}$ & $391-C-2-A$ & Balance & 10.64 & Normal & 0.17 & 1.62 & 1.70 \\
\hline Sleeve & $692-C-1-C$ & Balance & 10.05 & 93.14 & 0.12 & 1.65 & 1.70 \\
\hline End Sleeve & $630-A-4-B$ & Balance & 18.40 & 92.5 & 0.08 & 1.50 & 1.70 \\
\hline $\begin{array}{l}\mathrm{H}-2 \\
\text { Support Rod } \\
\text { Intermediate }\end{array}$ & $391-C-3-A$ & Balance & 10.64 & Normal & 0.17 & 1.62 & 1.70 \\
\hline Sleeve & $707-C-3-C$ & Balance & 10.56 & 93.05 & 0.12 & 1.65 & 1.71 \\
\hline End Sleeve & $630-A-4$ & Balance & 18.40 & 92.5 & 0.08 & 1.50 & 1.70 \\
\hline $\begin{array}{l}\text { H- } 3 \\
\text { Support Rod } \\
\text { Intermediate }\end{array}$ & $264-D-4-A$ & Balance & 10.46 & Normal & 0.13 & 1.65 & 1.71 \\
\hline $\begin{array}{l}\text { Sleeve } \\
\text { End Sleeve }\end{array}$ & $\begin{array}{l}707-D-2-C \\
630-A-6-A\end{array}$ & $\begin{array}{l}\text { Balance } \\
\text { Balance }\end{array}$ & $\begin{array}{l}10.52 \\
18.37\end{array}$ & $\begin{array}{l}93.05 \\
92.5\end{array}$ & $\begin{array}{l}0.13 \\
0.09\end{array}$ & $\begin{array}{l}1.63 \\
1.50\end{array}$ & $\begin{array}{l}1.70 \\
1.71\end{array}$ \\
\hline
\end{tabular}


TABLE 4 (Continuè

\begin{tabular}{|c|c|c|c|c|c|c|c|}
\hline \multirow{2}{*}{ Description } & \multirow{2}{*}{$\begin{array}{c}\text { Fuel } \\
\text { Rod } \\
\text { Number }\end{array}$} & \multirow{2}{*}{$\begin{array}{c}\text { Zirco- } \\
\text { nium } \\
(\text { wt } \%)\end{array}$} & \multicolumn{2}{|c|}{ Uranium } & \multirow{2}{*}{$\begin{array}{c}\text { Carbon } \\
(\text { wt } \%)\end{array}$} & \multicolumn{2}{|c|}{ Hydrogen } \\
\hline & & & $\begin{array}{l}\text { Total } \\
\text { (wt \%) }\end{array}$ & $\begin{array}{c}\text { Enrichment } \\
(\%)\end{array}$ & & (wt \%) & $\mathrm{H} / \mathrm{Zr}$ \\
\hline $\mathrm{H}-4$ & & & & & & & \\
\hline $\begin{array}{l}\text { Support Rod } \\
\text { Intermediate }\end{array}$ & $392-A-4-A$ & Balance & 10.62 & Normal & 0.12 & 1.78 & 1.85 \\
\hline Sleeve & $692-D-1-B$ & Balance & 9.98 & 93.11 & 0.15 & 1.79 & 1.85 \\
\hline End Sleeve & $630-A-6-A$ & Balance & 17.45 & 92.5 & 0.08 & 1.65 & 1.85 \\
\hline $\mathrm{H}-5$ & & & & & & & \\
\hline $\begin{array}{l}\text { Support Rod } \\
\text { Intermediate }\end{array}$ & $232-B-1-A$ & Balance & 10.14 & Normal & 0.18 & 1.75 & 1.83 \\
\hline Sleeve & $692-D-1-C$ & Balance & 9.98 & 93.11 & 0.15 & 1.78 & 1.85 \\
\hline End Sleeve & $630-A-9-B$ & Balance & 17.45 & 92.5 & 0.08 & 1.65 & 1.85 \\
\hline$M-1$ & & & & & & & \\
\hline $\begin{array}{l}\text { Support Rod } \\
\text { Intermediate }\end{array}$ & $391-C-3-C$ & Balance & 10.64 & Normal & 0.17 & 1.62 & 1.70 \\
\hline Sleeve & $707-D-2-B$ & Balance & 10.52 & 93.05 & 0.13 & 1.64 & 1.71 \\
\hline End Sleeve & $630-A-6-C$ & Balance & 18.37 & 92.5 & 0.09 & 1.50 & 1.71 \\
\hline $\begin{array}{l}\text { M-2 } \\
\text { Support Rod } \\
\text { Intermediate }\end{array}$ & $391-B-3-B$ & Balance & 10.65 & Normal & 0.15 & 1.64 & 1.71 \\
\hline Sleeve & $692-B-2-A$ & Balance & 10.08 & 93.12 & 0.14 & 1.65 & 1.72 \\
\hline End Sleeve & $630-A-6-A$ & Balance & 18.37 & 92.5 & 0.09 & 1.50 & 1.71 \\
\hline $\begin{array}{l}\text { M-3 } \\
\text { Support Rod } \\
\text { Intermediate }\end{array}$ & $310-A-1-B$ & Balance & 10.46 & Normal & 0.18 & 1.64 & 1.71 \\
\hline Sleeve & $692-C-2-A$ & Balance & 10.05 & 93.14 & 0.12 & 1.64 & 1.70 \\
\hline End Sleeve & $630-A-6$ & Balance & 18.37 & 92.5 & 0.09 & 1.50 & 1.71 \\
\hline$M-4$ & & & & & & & \\
\hline $\begin{array}{l}\text { Support Rod } \\
\text { Intermediate }\end{array}$ & $392-A-4-B$ & Balance & 10.62 & Normal & 0.14 & 1.78 & 1.86 \\
\hline Sleeve & $692-C-3-A$ & Balance & 10.05 & 93.14 & 0.12 & 1.78 & 1.85 \\
\hline End Sleeve & $630-A-5$ & Balance & 18.77 & 92.5 & 0.09 & 1.62 & 1.85 \\
\hline$M-5$ & & & & & & & \\
\hline $\begin{array}{l}\text { Support Rod } \\
\text { Intermediate }\end{array}$ & $392-A-1-B$ & Balance & 10.62 & Normal & 0.15 & 1.77 & 1.85 \\
\hline Sleeve & $692-D-1-A$ & Balance & 9.98 & 93.11 & 0.15 & 1.77 & 1.84 \\
\hline End Sleeve & $630-A-8$ & Balance & 18.55 & 92.5 & 0.11 & 1.50 & 1.70 \\
\hline $\mathrm{V}-1$ & & & & & & & \\
\hline $\begin{array}{l}\text { Support Rod } \\
\text { Intermediate }\end{array}$ & $391-B-4-A$ & Balance & 10.65 & Normal & 0.15 & 1.64 & 1.72 \\
\hline Sleeve & $707-D-2-A$ & Balance & 10.52 & 93.05 & 0.13 & 1.64 & 1.71 \\
\hline $\begin{array}{l}\text { End Sleeve } \\
V-2\end{array}$ & $630-A-6$ & Balance & 18.37 & 92.5 & 0.09 & 1.50 & 1.71 \\
\hline $\begin{array}{l}\text { Support Rod } \\
\text { Intermediate }\end{array}$ & $391-B-4-B$ & Balance & 10.65 & Normal & 0.15 & 1.64 & 1.71 \\
\hline Sleeve & $692-B-2-C$ & Balance & 10.08 & 93.12 & 0.14 & 1.65 & 1.71 \\
\hline End Sleeve & $630-A-3$ & Balance & 18.30 & 92.5 & 0.11 & 1.50 & 1.71 \\
\hline
\end{tabular}




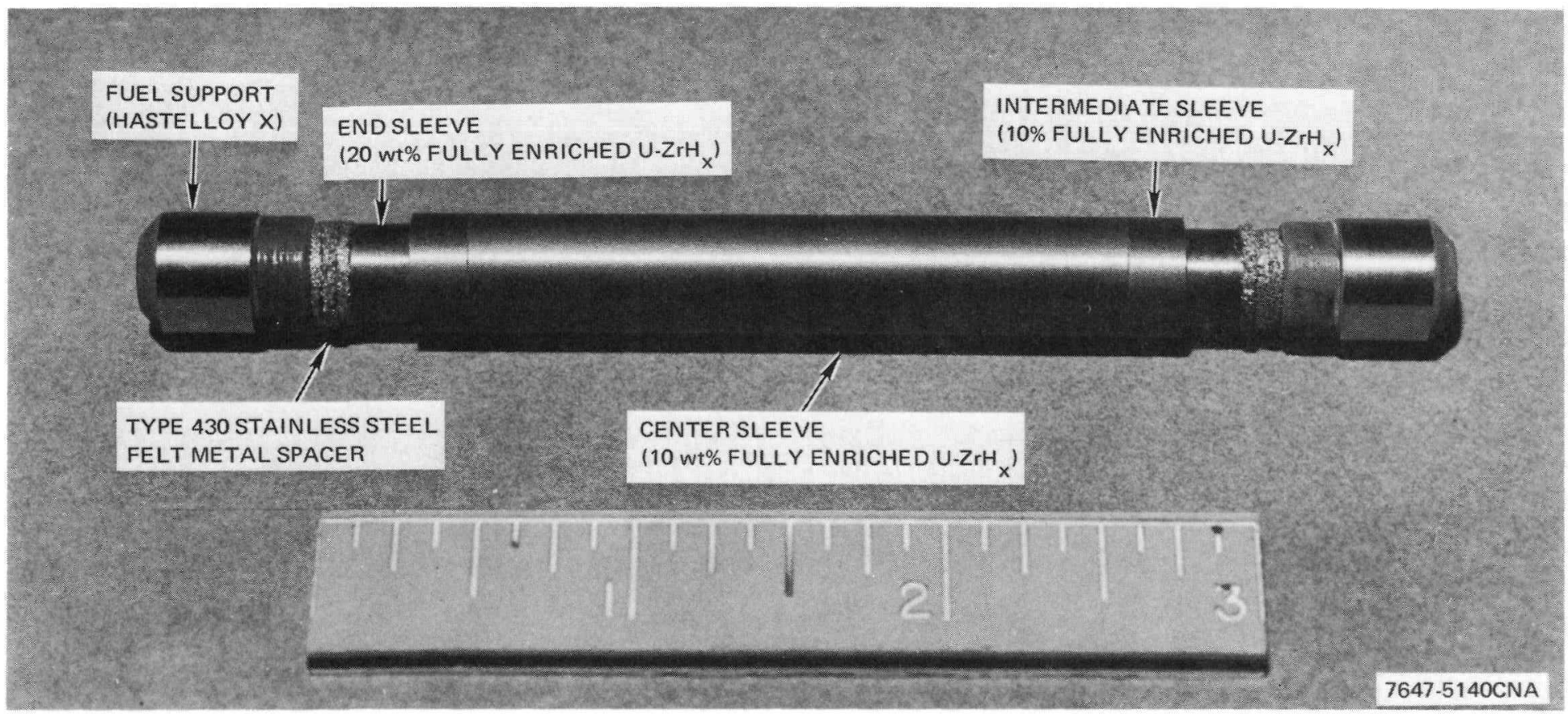

Figure 4. Typical NAA 120-4 Fuel Specimen Assembly (Figure Unclassified) 


\begin{tabular}{|c|c|c|c|c|c|c|c|c|c|c|c|c|c|c|c|c|c|c|c|c|c|}
\hline \multirow{2}{*}{$\begin{array}{c}\text { Sub- } \\
\text { capsule } \\
\text { Number }\end{array}$} & \multirow{2}{*}{$\begin{array}{l}\text { Specimen } \\
\text { Number }\end{array}$} & \multicolumn{3}{|c|}{$\begin{array}{l}\text { Support Rod } \\
\text { (in.) }\end{array}$} & \multicolumn{3}{|c|}{$\begin{array}{l}\text { End Fuel Sleeve } \\
\text { (in.) }\end{array}$} & \multicolumn{3}{|c|}{$\begin{array}{l}\text { Intermediate Fuel Sleeve } \\
\text { (in.) }\end{array}$} & \multicolumn{3}{|c|}{$\begin{array}{c}\text { Center Fuel Sleeve } \\
\text { (in.) }\end{array}$} & \multicolumn{3}{|c|}{$\begin{array}{l}\text { Intermediate Fuel Sleeve } \\
\text { (in.) }\end{array}$} & \multicolumn{3}{|c|}{$\begin{array}{l}\text { End Fuel Sleeve } \\
\text { (in.) }\end{array}$} & \multicolumn{2}{|c|}{$\begin{array}{l}\text { Fuel-Cladding } \\
\text { As-Assembled } \\
\text { Clearance } \\
\text { (mils) }\end{array}$} \\
\hline & & Length & OD & ID & Length & OD & ID & Length & OD & ID & Length & OD & ID & Length & OD & ID & Length & OD & ID & Radial & Axial \\
\hline \multirow{5}{*}{1} & $E-1$ & 3.747 & 0.2502 & 0.098 & 0.1987 & $|0.3352|$ & 0.2502 & 0.1991 & 0.4092 & 0.2503 & 2.251 & 0.4093 & 0.2506 & 0.1993 & 0.4093 & 0.2503 & 0.1993 & 0.3369 & 0.2501 & $4.2 \pm 1.4$ & 80 \\
\hline & $E-2$ & 3.753 & 0.2501 & - & 0.1995 & 0.3319 & 0.2501 & 0.1985 & 0.4091 & 0.2501 & 2.250 & 0.4092 & 0.2504 & 0.1986 & 0.4090 & 0.2500 & 0.1988 & 0.3354 & 0.2500 & $4.2 \pm 0.8$ & 50 \\
\hline & $E-3$ & 3.752 & 0.2501 & - & 0.1914 & 0.3359 & 0.2500 & 0.1988 & 0.4090 & 0.2502 & 2.250 & 0.4090 & 0.2503 & 0.1988 & 0.4088 & 0.2501 & 0.1988 & 0.3357 & 0.2499 & $4.4 \pm 1.3$ & 90 \\
\hline & $E-4$ & 3.752 & 0.2502 & - & 0.1988 & 0.3363 & 0.2501 & 0.1998 & 0.4087 & 0.2502 & 2.249 & 0.4093 & 0.2503 & 0.1998 & 0.4092 & 0.2503 & 0.1998 & 0.3363 & 0.2503 & $4.1 \pm 1.2$ & 37 \\
\hline & $E-5$ & 3.754 & 0.2503 & - & 0.1995 & 0.3373 & 0.2508 & 0.1999 & 0.4090 & 0.2502 & 2.250 & 0.4091 & 0.2505 & 0.2003 & 0.4092 & 0.2503 & 0.1993 & 0.3350 & 0.2502 & $4.2 \pm 0.8$ & 88 \\
\hline \multirow{5}{*}{2} & $\mathrm{H}-1$ & 3.755 & 0.2500 & 0.098 & 0.1991 & 0.3312 & 0.2499 & 0.198 & 0.4011 & 0.2500 & 2.250 & 0.4011 & 0.2500 & 0.198 & 0.4012 & 0.2500 & 0.1986 & 0.3310 & 0.2499 & $8.1 \pm 1.5$ & 129 \\
\hline & $\mathrm{H}-2$ & 3.752 & 0.2502 & - & 0.1979 & 0.3303 & 0.2502 & 0.1992 & 0.4010 & 0.2500 & 2.250 & 0.4007 & 0.2502 & 0.1993 & 0.4009 & 0.2502 & 0.1923 & 0.3303 & 0.2501 & $8.2 \pm 1.6$ & 44 \\
\hline & $\mathrm{H}-3$ & 3.752 & 0.2501 & - & 0.2083 & 0.3307 & 0.2505 & 0.2002 & 0.4010 & 0.2500 & 2.249 & 0.4011 & 0.2501 & 0.2003 & 0.4011 & 0.2502 & 0.1998 & 0.3307 & 0.2499 & $8.4 \pm 1.3$ & 75 \\
\hline & $\mathrm{H}-4$ & 3.752 & 0.2502 & - & 0.1991 & 0.3305 & 0.2501 & 0.1992 & 0.4011 & 0.2502 & 2.248 & 0.4010 & 0.2505 & 0.1991 & 0.4011 & 0.2502 & 0.1998 & 0.3306 & 0.2501 & $8.4 \pm 1.3$ & 54 \\
\hline & $\mathrm{H}-5$ & 3.752 & 0.2502 & - & 0.1991 & 0.3302 & 0.2500 & 0.1992 & 0.4011 & 0.2501 & 2.254 & 0.4011 & 0.2503 & 0.1978 & 0.4009 & 0.2501 & 0.1972 & 0.3303 & 0.2501 & $8.4 \pm 1.6$ & 28 \\
\hline \multirow{5}{*}{3} & $M-1$ & 3.750 & 0.2501 & 0.098 & 0.1991 & 0.3311 & 0.2499 & 0.2013 & 0.3920 & 0.2499 & 2.251 & 0.3921 & 0.2502 & 0.1994 & 0.3924 & 0.2499 & 0.1996 & 0.3307 & 0.2501 & $12.6 \pm 0.8$ & 110 \\
\hline & M-2 & 3.752 & 0.2501 & - & 0.1990 & 0.3303 & 0.2499 & 0.1993 & 0.3920 & 0.2502 & 2.249 & 0.3921 & 0.2503 & 0.1991 & 0.3921 & 0.2501 & 0.1993 & 0.3307 & 0.2499 & $12.9 \pm 1.8$ & 76 \\
\hline & $M-3$ & 3.752 & 0.2500 & - & 0.1998 & 0.3307 & 0.2499 & 0.1999 & 0.3917 & 0.2500 & 2.254 & 0.3921 & 0.2501 & 0.1996 & 0.3920 & 0.2501 & 0.1998 & 0.3307 & 0.2499 & $13.0 \pm 1.9$ & 93 \\
\hline & M-4 & 3.751 & 0.2503 & - & 0.1989 & 0.3305 & 0.2501 & 0.2001 & 0.3919 & 0.2501 & 2.251 & 0.3921 & 0.2503 & 0.1991 & 0.3922 & 0.2503 & 0.1991 & 0.3305 & 0.2501 & $12.8 \pm 1.8$ & 85 \\
\hline & M-5 & 3.751 & 0.2502 & - & 0.1962 & 0.3305 & 0.2508 & 0.1984 & 0.3922 & 0.2501 & 2.249 & 0.3922 & 0.2502 & 0.1994 & 0.3922 & 0.2501 & 0.1967 & 0.3302 & 0.2502 & $12.9 \pm 2.4$ & 43 \\
\hline 4 & $v-1$ & 3.754 & 0.2500 & 0.098 & 0.1994 & 0.3308 & 0.2499 & 0.1998 & 0.4014 & 0.2500 & 2.249 & 0.4012 & 0.2501 & 0.2002 & 0.4011 & 0.2499 & 0.1989 & 0.3309 & 0.2499 & $8.0 \pm 1.2$ & 89 \\
\hline 5 & $v-2$ & 3.751 & 0.2501 & 0.098 & 0.2003 & 0.3307 & 0.2499 & 0.1998 & 0.4007 & 0.2500 & 2.251 & 0.4011 & 0.2501 & 0.2002 & 0.4007 & 0.2500 & 0.1983 & 0.3307 & 0.2499 & $8.0 \pm 1.1$ & 76 \\
\hline
\end{tabular}

Table 5. As-Built Dimensions

of Test Specimen Suppor 
BLANK 


\section{FUEL SPECIMEN ASSEMBLY}

Final fabrication of the fuel specimen assemblies consisted of: (1) insertion of the fuel assembly into the cladding tube assembly, (2) as sembly and welding of the lower end cap into the tube, and (3) performance of the ceramic-to-ceramic fusion of the lower end cap to the tube.

Permeation measurements were made by placing the completed assembly in a system evacuated to $10^{-4}$ torr. A mass spectrometer was used to measure hydrogen loss rates at appropriate isothermal test temperatures. Table 6 shows the results of these tests. Those specimens which did not meet the acceptance criteria were subsequently permation scanned, with the exception of Specimen E-1. This specimen, as well as the other instrumented specimens, had an uncoated pressure sensor tube which, under in-pile conditions, would operate at a much lower temperature, and hence lower permeation rate. When the calculated bare metal permeation loss through the probe was subtracted from the $1250^{\circ} \mathrm{F}$ isothermal permeation rate, the specimen was found to be acceptable.

Ten fuel specimen assemblies exhibited isothermal permeation rates that were higher than acceptable. These specimens were subjected to permeation scan tests. The scanning tests showed that the hydrogen permeating the center 4-1/4 in. of the specimen tube amounted to a maximum of $10 \%$ of the total. Since the ends were to operate at a lower temperature during in-pile testing, resulting in lower hydrogen loss rates, the specimens were considered acceptable. (18) The results of the hydrogen permeation scanning tests are also shown in Table 6. 
TABLE 6

FUEL SPECIMEN PERMEATION RATES AND SCANNING TEST RESULTS

\begin{tabular}{|c|c|c|c|c|c|c|c|}
\hline \multirow{3}{*}{$\begin{array}{l}\text { Assembly } \\
\text { Number }\end{array}$} & \multicolumn{4}{|c|}{$\begin{array}{l}\text { Hydrogen Permeation Rate } \\
{[\mathrm{cc}(\mathrm{STP}) / \mathrm{hr}]}\end{array}$} & \multicolumn{3}{|c|}{$\begin{array}{c}\text { Permeation Scan Tests } \\
\text { (\% hydrogen loss at) }\end{array}$} \\
\hline & \multicolumn{3}{|c|}{$\begin{array}{c}\text { Isothermal Test } \\
\text { Temperature } \\
\left({ }^{\circ} \mathrm{F}\right)\end{array}$} & \multirow{2}{*}{$\begin{array}{c}\text { Maximum } \\
\text { Allowable } \\
1250^{\circ} \mathrm{F} \\
\text { Rate }\end{array}$} & \multirow[t]{2}{*}{ Blind } & \multirow[t]{2}{*}{ Center $\S$} & \multirow[t]{2}{*}{$\underset{\text { End }}{\text { Closure }}$} \\
\hline & 1100 & 1200 & 1250 & & & & \\
\hline$E-1$ & 0.19 & 0.61 & $1.6 *$ & 1.03 & - & - & - \\
\hline$E-2$ & 0.03 & 0.04 & 0.09 & 0.45 & - & - & - \\
\hline$E-3$ & 0.03 & 0.08 & 0.11 & 0.45 & - & - & - \\
\hline$E-4$ & 0.11 & 0.24 & 0.44 & 1.03 & - & - & - \\
\hline$E-5$ & 0.08 & 0.26 & 0.33 & 1.03 & - & - & - \\
\hline $\mathrm{H}-1$ & 0.11 & 0.33 & 0.67 & 0.10 & 52 & 1 & 48 \\
\hline $\mathrm{H}-2$ & 0.05 & 0.13 & 0.71 & 0.10 & 50 & 1 & 50 \\
\hline $\mathrm{H}-3$ & 0.01 & 0.01 & 0.04 & 0.10 & - & - & - \\
\hline $\mathrm{H}-4$ & 0.02 & 0.05 & 0.23 & 0.26 & - & - & - \\
\hline $\mathrm{H}-5$ & 0.02 & 0.14 & 0.28 & 0.26 & 82 & 1 & 18 \\
\hline$M-1$ & 0.13 & 0.54 & 1.04 & 0.05 & 52 & 1 & 48 \\
\hline$M-2$ & 0.06 & 0.24 & 0.47 & 0.05 & 54 & 1 & 46 \\
\hline $\mathrm{M}-3$ & 0.06 & 0.24 & 0.47 & 0.05 & 47 & 1 & 53 \\
\hline$M-4$ & 0.04 & 0.14 & 0.26 & 0.10 & 52 & 4 & 44 \\
\hline$M-5$ & 0.03 & 0.12 & 0.26 & 0.10 & 46 & 11 & 43 \\
\hline V- 1 & 0.14 & 0.57 & 1.02 & 0.10 & 54 & 1 & 46 \\
\hline$V-2$ & 0.20 & 0.77 & 1.17 & 0.10 & 58 & 1 & 42 \\
\hline
\end{tabular}

*E- 1 considered acceptable, based on calculation of hydrogen loss attributable to ends and thermocouple well.

†Includes end-cap and 1/2-in. of cladding.

\$Center 4-1/4 in. of cladding. 


\section{OPERATION}

As stated previously, the NAA 120-4 was irradiated in the $2 \mathrm{~A}$ Test Facilit in the HAPO KE Reactor. The temperatures of each of the five subassemblies were independently controlled, through the use of a binary (helium and argon) control system. A variable mixture of helium and argon was sent from ex-reactor gas supplies, through the control system, into annuli concentric with the outsine of the fuel specimens. Each experiment subassembly was controlled, using the fuel centerline thermocouple in the instrumented fuel specimen. The fuel temperature was maintained at design temperature conditions, whenever possible, by altering the binary control gas mixture. Cladding temperatures were not maintained at any specified level, but were not allowed to exceed $1300^{\circ} \mathrm{F}$ for any long periods, due to cladding strength considerations.

The data recording and monitoring system ${ }^{(19)}$ provided a punched paper tape record of all the thermocouple readings, with a strip chart record on most of the thermocouples for direct readout purposes. Each of the thermocouples had high- and low-alarm limit input values, which provided an automatic helium purge of the capsule gas annulus to prevent over-temperature operation.

Startup and operation of the experiment proceeded as planned. (20) After $\sim 1500 \mathrm{hr}$ of experiment operation, Fuel Specimen M-1 could not be maintained at design operating conditions $\left(1600^{\circ} \mathrm{F}\right.$ fuel centerline temperature), even with pure argon in the gas control annulus. This was due to a decrease in its fuel-tocladding $\$ \mathrm{~T}$, which had occurred much more rapidly than had been anticipated. The othe specimens instrumented with in-fuel thermocouples showed similar decrease in fuel-to-cladding $\Delta \mathrm{T}$, but proportionally smaller, due to their lower design full temperatures. Power-normalized fuel-to-cladding $\Delta \mathrm{T}^{\prime} \mathrm{s}$ as a function of time attemperature are shown in Figure 5.

After $2100 \mathrm{hr}$ at operating temperature, the instrumentation indicated that a signific nt amount of fission gas had been released into the fuel-to-cladding hydrogen gap of the 1500 and $1600^{\circ} \mathrm{F}$ fuel specimens (pressure rise and fuel-tocladding ${ }^{\mathrm{s} T}$ increase). Similar indications were seen in the $1300^{\circ} \mathrm{F}$ fuel specimen (E-I) after $\sim 6000 \mathrm{hr}$. In all cases, the fission gas was released while the experiment was at helium operating conditions, and was probably due to the 20 wt $\%$ end fuel sleeves changing phase. 


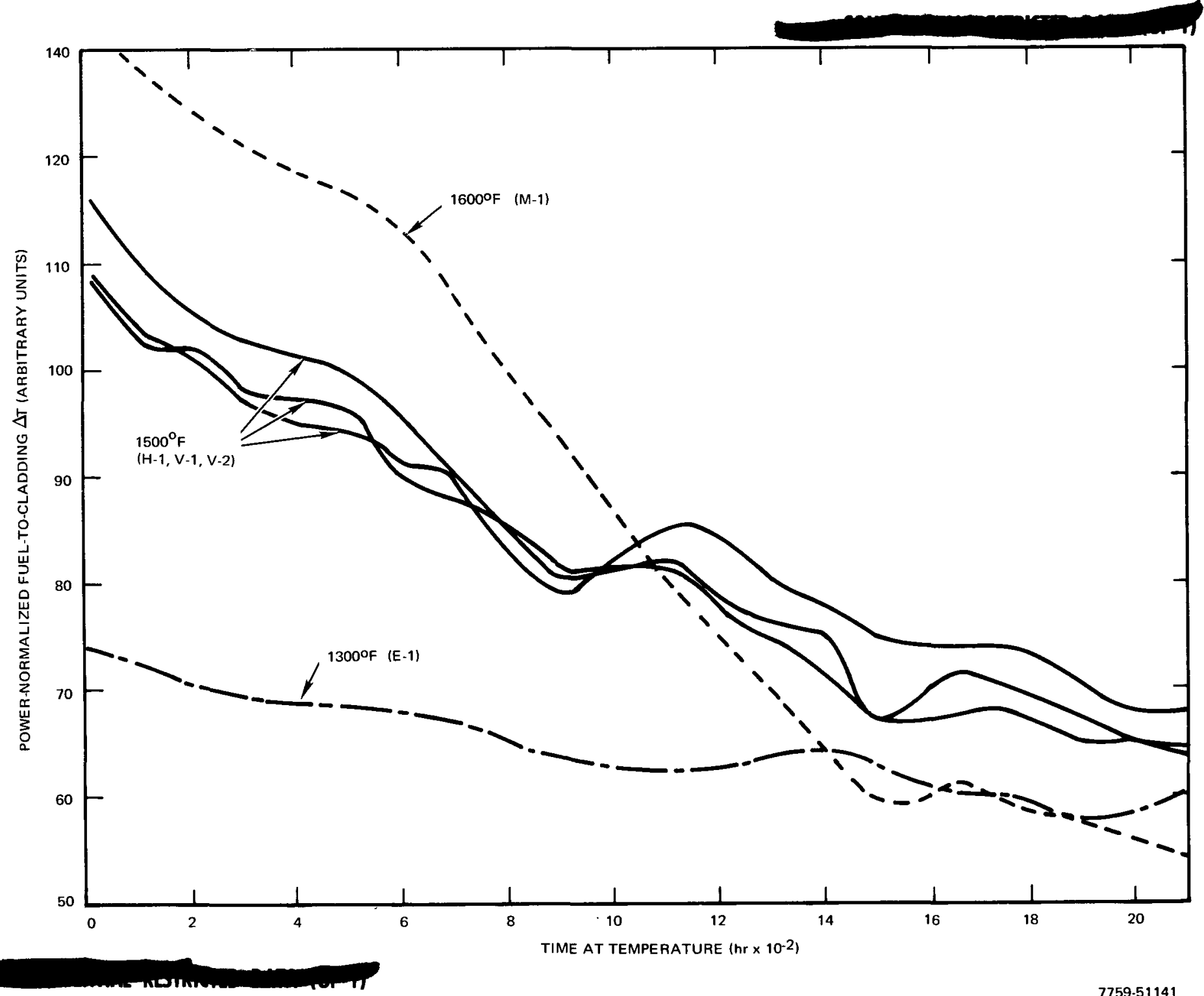

Figure 5. Power-Normalized Fuel-toCladding $\Delta \mathrm{T}$ vs Time (Average data) 
Although the phase change experiments ${ }^{(8)}$ had been planned for Specimens $\mathrm{V}-1$ and V-2, only Specimen V-2 was intentionally driven into $\beta$ phase. This was due to a failure of a weld joint on Specimen $V-1$, and subsequent $\mathrm{NaK}$ inleakage into the cladding, which plugged the pressure line to the specimen.

Time-averaged operating temperatures are listed in Table $7 .^{(21)}$ During the final shutdown of the experiment, temperatures were decreased as rapidly as possible from design operating conditions, so that the hydrogen profile in the fuel would reflect the operating hydrogen profile.

Upon reaching a calculated burnup ranging from 0.82 to 1.03 metal at. \%, the experiment was discharged from the reactor. At the time of discharge, all but two cladding thermocouples, and all in-fuel thermocouples, were in operating condition. 
TABLE 7

TIME-AVERAGED OPERATING TEMPERATURES

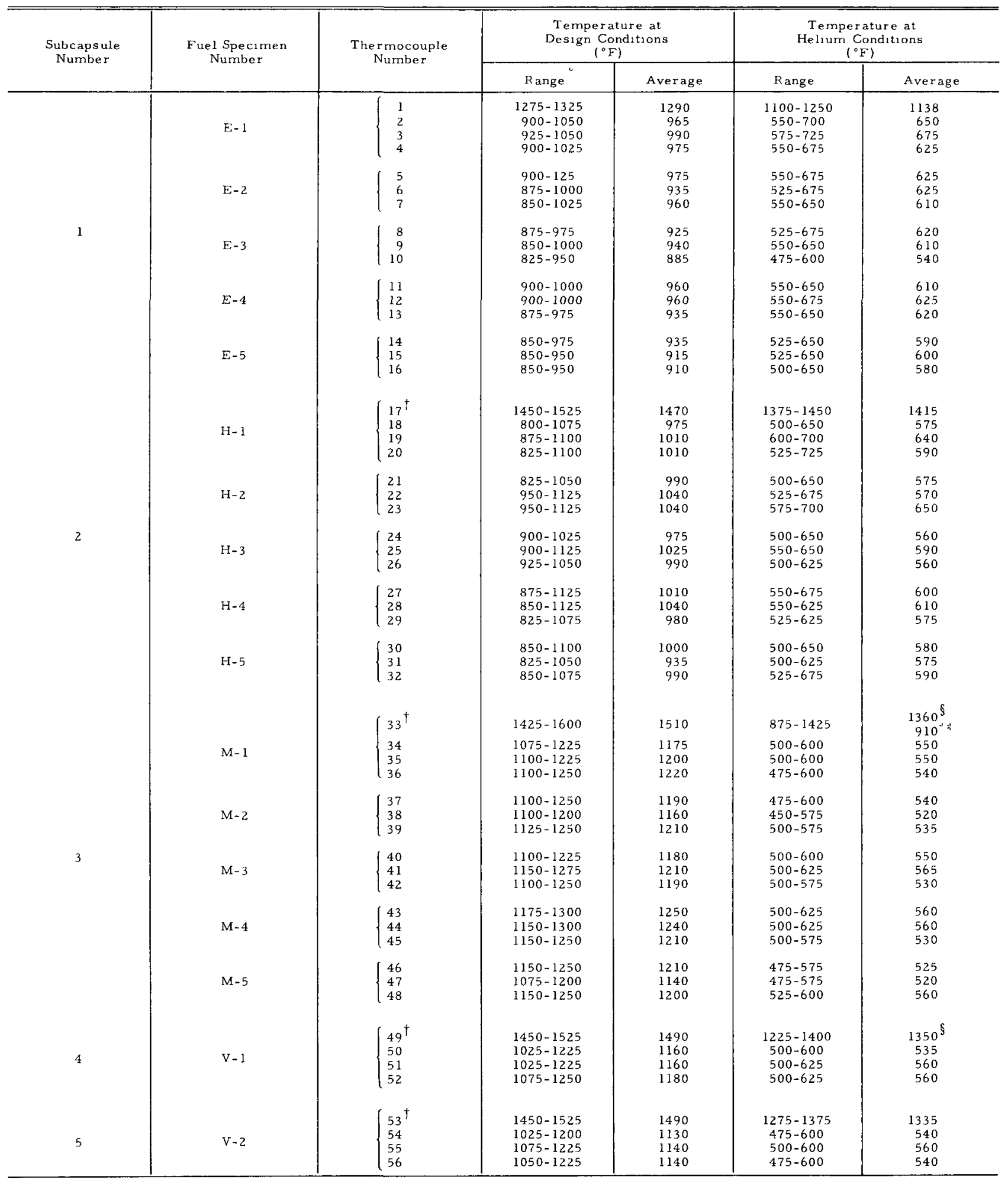

Range is considered the temperature domain in which the thermocouple spent the majority of the time In-fuel thermocouple

§arly in life

Late in life

AI-AEC- 12963

28 


\section{POSTIRRADIATION EXAMINATION}

\section{A. EXPERIMENT DISASSEMBLY AND EXAMINATION}

All five subcapsules were visually examined, and appeared in excellent condition after the test. (22) The subcapsules were then cut open, and the fuel assemblies removed and cleaned. Examination of the fuel assemblies, using both Kollmorgen periscope and stereomicroscope, showed that all thermocouples, bands, dosimeters, and all related components were in excellent condition.

\section{B. FUEL SPECIMEN ASSEMBLIES}

Disassembly of the fuel element assemblies showed $\mathrm{NaK}$ weeping from the gas line in Specimen V-1. Subsequent examination showed two small pinholes in the gas line weld area at the upper end cap of the element, which permitted $\mathrm{NaK}$ to enter the element cladding.

Neutron radiography proved to be a successful method to examine irradiated fuel elements nondestructively. In most cases, it indicated the fuel specimen condition and a relative hydrogen distribution. In some cases, because of fuel condition, dimensional measurements of the fuel were deduced from the radiographs. This type of examination aided subsequent disassembly procedures and sample selection.

As can be seen in Figure 6, the neutron radiographs show that a good number of the support rods (normal U) were broken off, and remained inside the Hastelloy $X$ fuel supports. No absolute value was assigned to the hydrogen content, although a relative hydrogen distribution within a specimen is clearly indicated, by comparison of the radiographs and the hydrogen analysis reported later in Table 12. The radiographs also indicated that the support rods inside the Hastelloy $X$ fuel supports had a higher hydrogen content than did other portions of the fuel specimen (note the darker color in Figure 6).

A series of diameter and length measurements were made on each of the clad fuel elements, with dial indicators and vernier calipers. The accuracy of the dial indicators, when used remotely, is $\pm 0.001 \mathrm{in.}$, while the accuracy of the vernier calipers is $\pm 0.0015 \mathrm{in}$. The results of these measurements are shown in Table 8. The length measurements showed no significant changes. The 


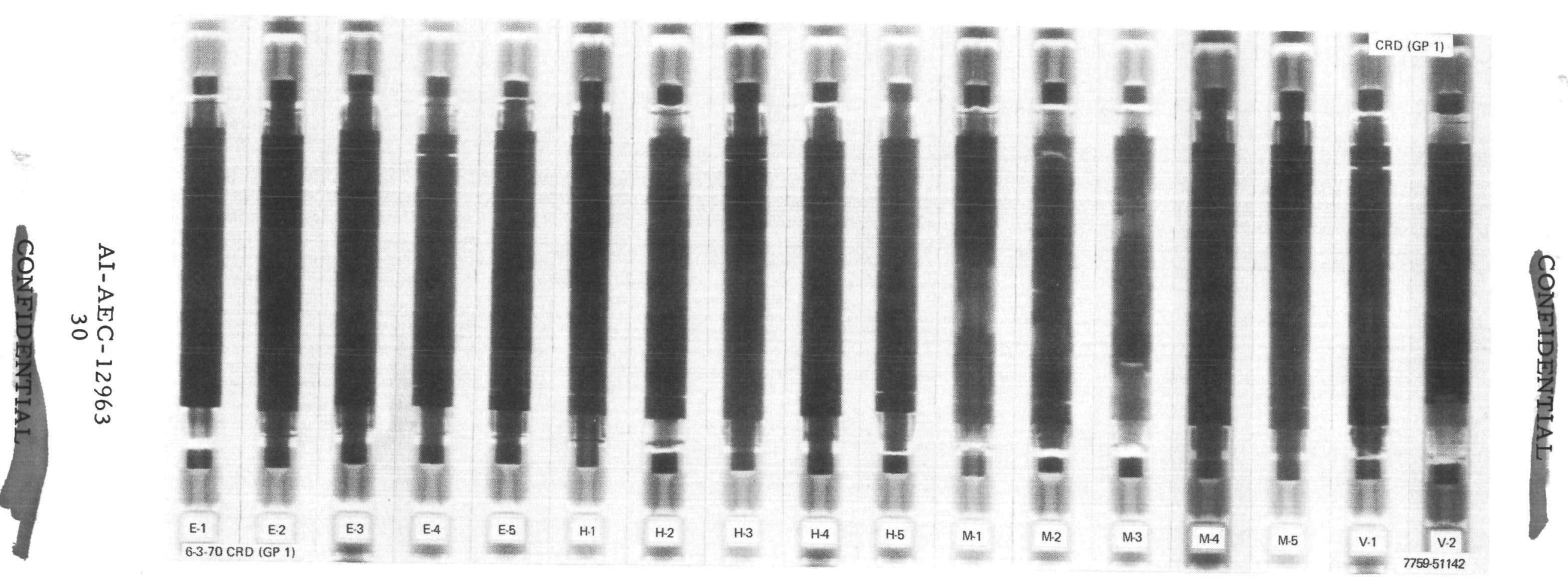

Figure 6. Neutron Radiographs of the NAA 120-4 Experiment 
TABLE 8

NAA 120-4 FUEL SPECIMEN CLADDING DIMENSIONAL MEASUREMENTS

\begin{tabular}{|c|c|c|c|c|c|c|}
\hline $\begin{array}{l}\text { Element } \\
\text { Number }\end{array}$ & $\begin{array}{c}\text { Average } \\
\text { Pre-Irradiation } \\
\text { Diameter } \\
\text { Measurements } \\
\text { (in.) }\end{array}$ & $\begin{array}{c}\text { Average } \\
\text { Postirradiation } \\
\text { Diameter } \\
\text { Measurements } \\
\text { (in.) }\end{array}$ & $\begin{array}{c}\text { Average } \\
\Delta D \\
(\text { in. })\end{array}$ & $\begin{array}{c}\text { Average } \\
\text { Pre-Irradiation } \\
\text { Length } \\
\text { Measurements } \\
\text { (in.) }\end{array}$ & $\begin{array}{c}\text { Average } \\
\text { Postirradiation } \\
\text { Length } \\
\text { Measurements } \\
\text { (in.) }\end{array}$ & $\begin{array}{c}\text { Average } \\
\Delta L \\
\text { (in.) }\end{array}$ \\
\hline $\begin{array}{l}E-1 \\
E-2 \\
E-3 \\
E-4 \\
E-5\end{array}$ & $\begin{array}{l}0.4813 \\
0.4817 \\
0.4817 \\
0.4818 \\
0.4813\end{array}$ & $\begin{array}{l}0.4811 \\
0.4815 \\
0.4814 \\
0.4831 \\
0.4812\end{array}$ & $\begin{array}{l}-0.0002 \\
-0.0002 \\
-0.0003 \\
+0.0013 \\
-0.001\end{array}$ & $\begin{array}{l}5.246 \\
5.243 \\
5.246 \\
5.246 \\
5.247\end{array}$ & $\begin{array}{l}5.245 \\
5.242 \\
5.245 \\
5.247 \\
5.246\end{array}$ & $\begin{array}{l}-0.001 \\
-0.001 \\
-0.001 \\
+0.001 \\
-0.001\end{array}$ \\
\hline $\begin{array}{l}\mathrm{H}-1 \\
\mathrm{H}-2 \\
\mathrm{H}-3 \\
\mathrm{H}-4 \\
\mathrm{H}-5\end{array}$ & $\begin{array}{l}0.4816 \\
0.4813 \\
0.4813 \\
0.4805 \\
0.4809\end{array}$ & $\begin{array}{l}0.4810 \\
0.4809 \\
0.4807 \\
0.4803 \\
0.4808\end{array}$ & $\begin{array}{l}-0.0006 \\
-0.0004 \\
-0.0006 \\
-0.0002 \\
-0.0001\end{array}$ & $\begin{array}{l}5.245 \\
5.245 \\
5.246 \\
5.246 \\
5.247\end{array}$ & $\begin{array}{l}5.245 \\
5.245 \\
5.245 \\
5.244 \\
5.245\end{array}$ & $\begin{array}{r}0.000 \\
0.000 \\
-0.001 \\
-0.002 \\
-0.002\end{array}$ \\
\hline $\begin{array}{l}M-1 \\
M-2 \\
M-3 \\
M-4 \\
M-5\end{array}$ & $\begin{array}{l}0.4814 \\
0.4813 \\
0.4815 \\
0.4812 \\
0.4813\end{array}$ & $\begin{array}{l}0.4817 \\
0.4814 \\
0.4823 \\
0.4810 \\
0.4810\end{array}$ & $\begin{array}{l}+0.0003 \\
+0.0001 \\
+0.0008 \\
-0.0002 \\
-0.0003\end{array}$ & $\begin{array}{l}5.250 \\
5.238 \\
5.246 \\
5.245 \\
5.245\end{array}$ & $\begin{array}{l}5.249 \\
5.239 \\
5.248 \\
5.246 \\
5.246\end{array}$ & $\begin{array}{l}-0.001 \\
+0.001 \\
+0.002 \\
+0.001 \\
+0.001\end{array}$ \\
\hline $\begin{array}{l}V-1 \\
V-2\end{array}$ & $\begin{array}{l}0.4812 \\
0.4811\end{array}$ & $\begin{array}{l}0.4809 \\
0.4812\end{array}$ & $\begin{array}{l}-0.0003 \\
+0.0001\end{array}$ & $\begin{array}{l}5.246 \\
5.236\end{array}$ & $\begin{array}{l}5.245 \\
5.236\end{array}$ & $\begin{array}{r}-0.001 \\
0.000\end{array}$ \\
\hline
\end{tabular}


cladding diameter measurements generally indicated little or no change between pre- and post-average diameters, although some postirradiation measurements did indicate fuel-cladding interference. Also, the postirradiation diameter cladding measurements of $\mathrm{H}-2, \mathrm{H}-3, \mathrm{H}-4$, and $\mathrm{H}-5$ showed smaller diameters at the top half of the specimen than at the lower half. The difference amounted to $\sim 0.001$ in.

Examination after fuel specimen disassembly, summarized later in Table 14, showed cracking in Fuel Specimens E-4, H-2, H-5, M-1, M-2, M-3, V-1, and

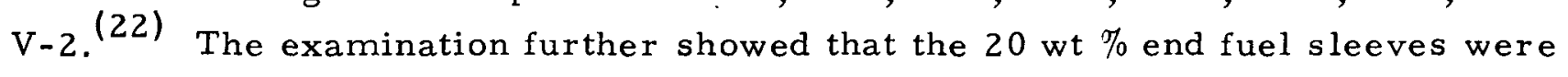
badly swollen, cracked, and/or broken, in all but one specimen assembly (M-4). Seven of the 17 specimen assemblies were damaged to some degree in the throwaway 10 wt $\%$ end sleeves. The damaged condition of the 20 wt $\%$, and some of the $10 \mathrm{wt} \%$, end sleeves was expected.

Nine of the support rods could not be removed from their fuel sleeves. All of the in-fuel thermocouples and their respective thermocouple wells were easily removed from the support rods, except for Specimen V-1. The in-fuel thermocouple and its container in V-1 snapped off just below the fuel support during handling, and appeared very brittle. The portion that was in the support rod remained stuck. Reaction was observed on all support rods under the 20 wt $\%$ fuel sleeves; and, in some cases, at the interface between the 10 and $20 \mathrm{wt} \%$ end fuel sleeves. The reaction on the support and sleeves is thought to be a result of a eutectic which formed between the Type 430 stainless steel Feltmetal and the 20 wt $\%$ end fuel sleeves. The temperature needed to form a eutectic between these two alloys is $\sim 1340^{\circ} \mathrm{F}$, and the $20 \mathrm{wt} \%$ fuel sleeves operated above this temperature.

In most cases, the support rod was broken off in the fuel supports; and, in no case, could the support rod be removed from the fuel support. This is thought to be due to local diameter increases in the cold ends, caused by hydrogen redistribution.

Figures 7, 8, and 9 show photographs of representative fuel assemblies, taken during examination of the experiment. Specimen E-1 (Figure 7) is representative of specimens which were intact following the test. Specimens $M-1$ and E-4 (Figures 8 and 9) are representative of cracked and broken fuel assemblies, 


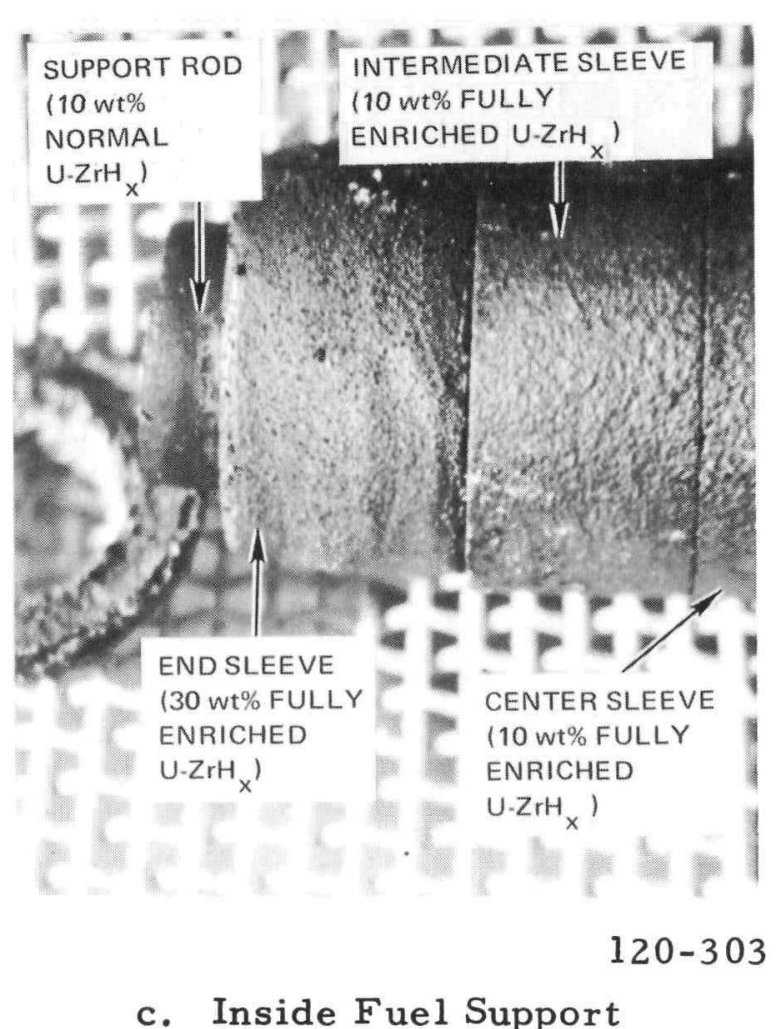

c. Inside Fuel Suppor

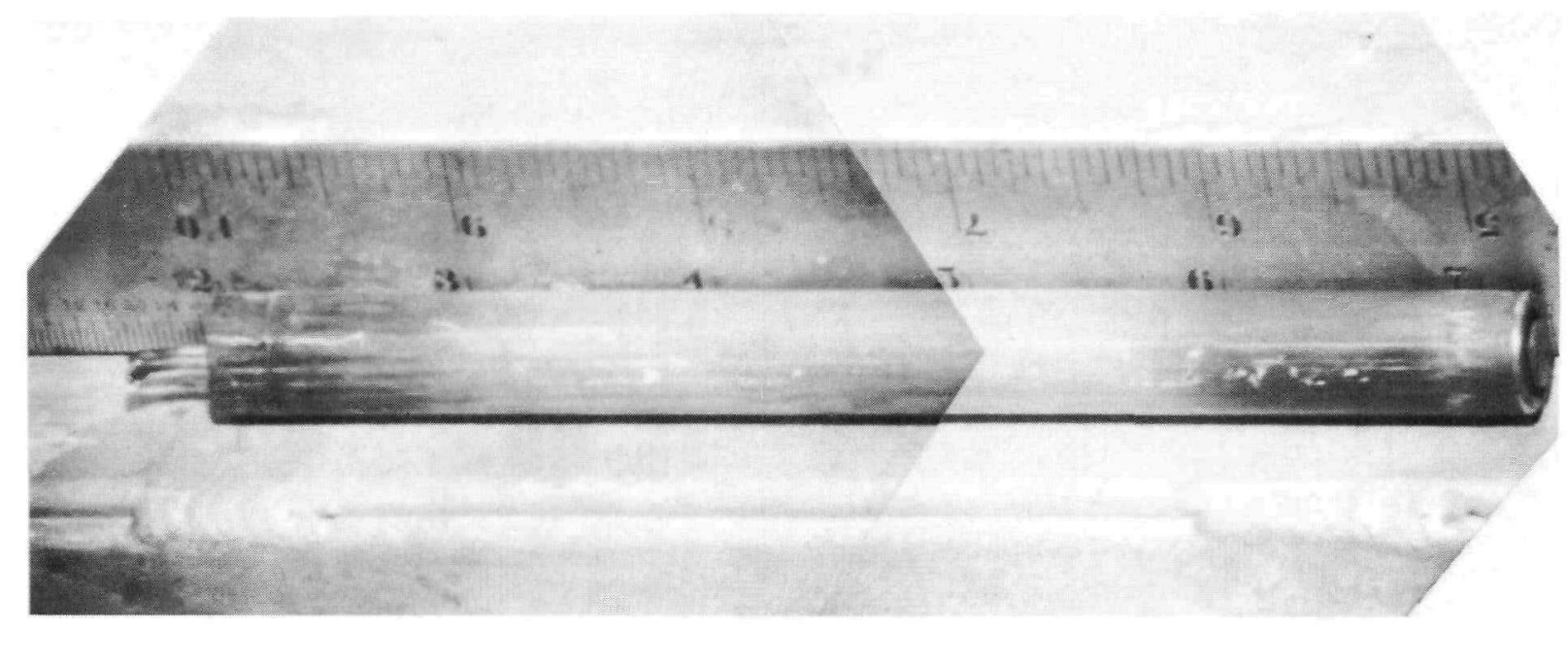

a. Before Disassembly

$120-156,-157$

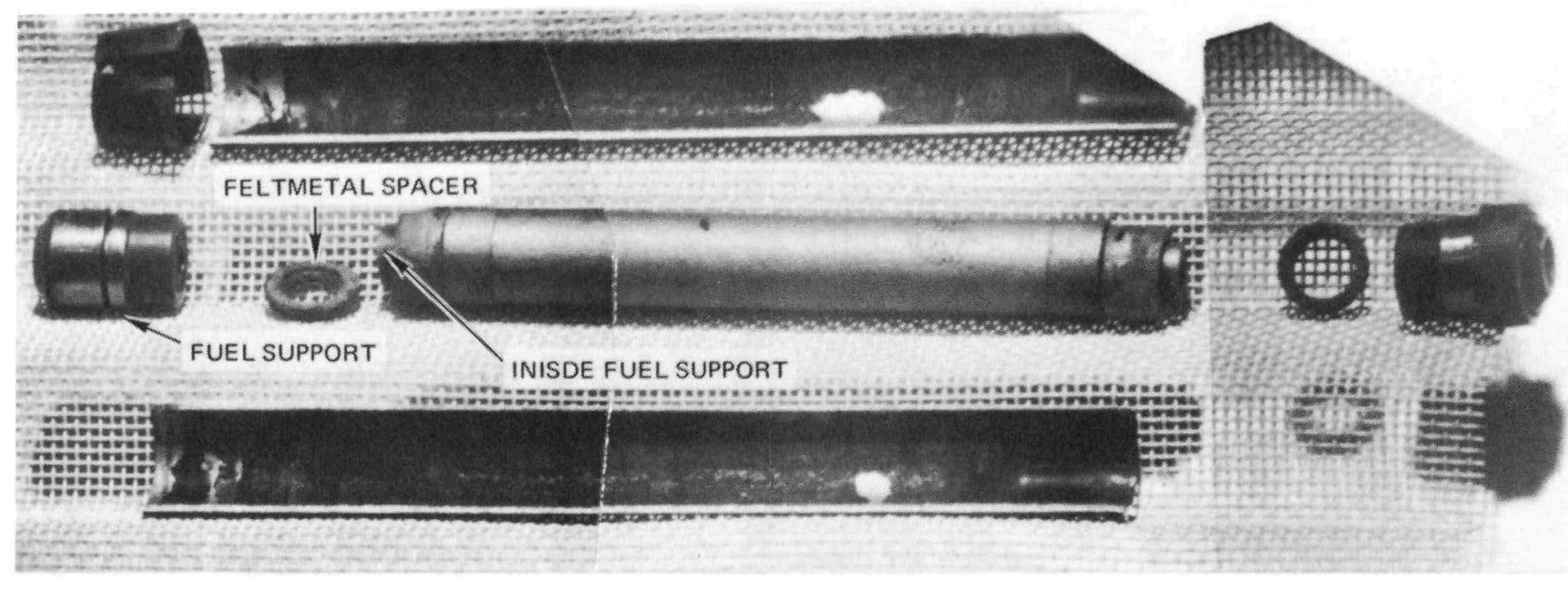

b. After Disassembly

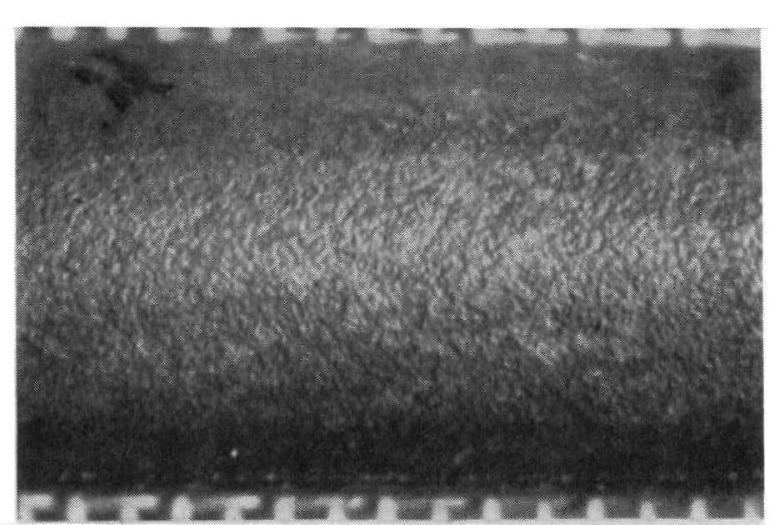

120-302

d. Typical Area of Center Fuel Sleeve
Figure 7. Specimen E-1 (Figure Unclassified) 
BLANK 




$120-238,-239$

a. Before Disassembly

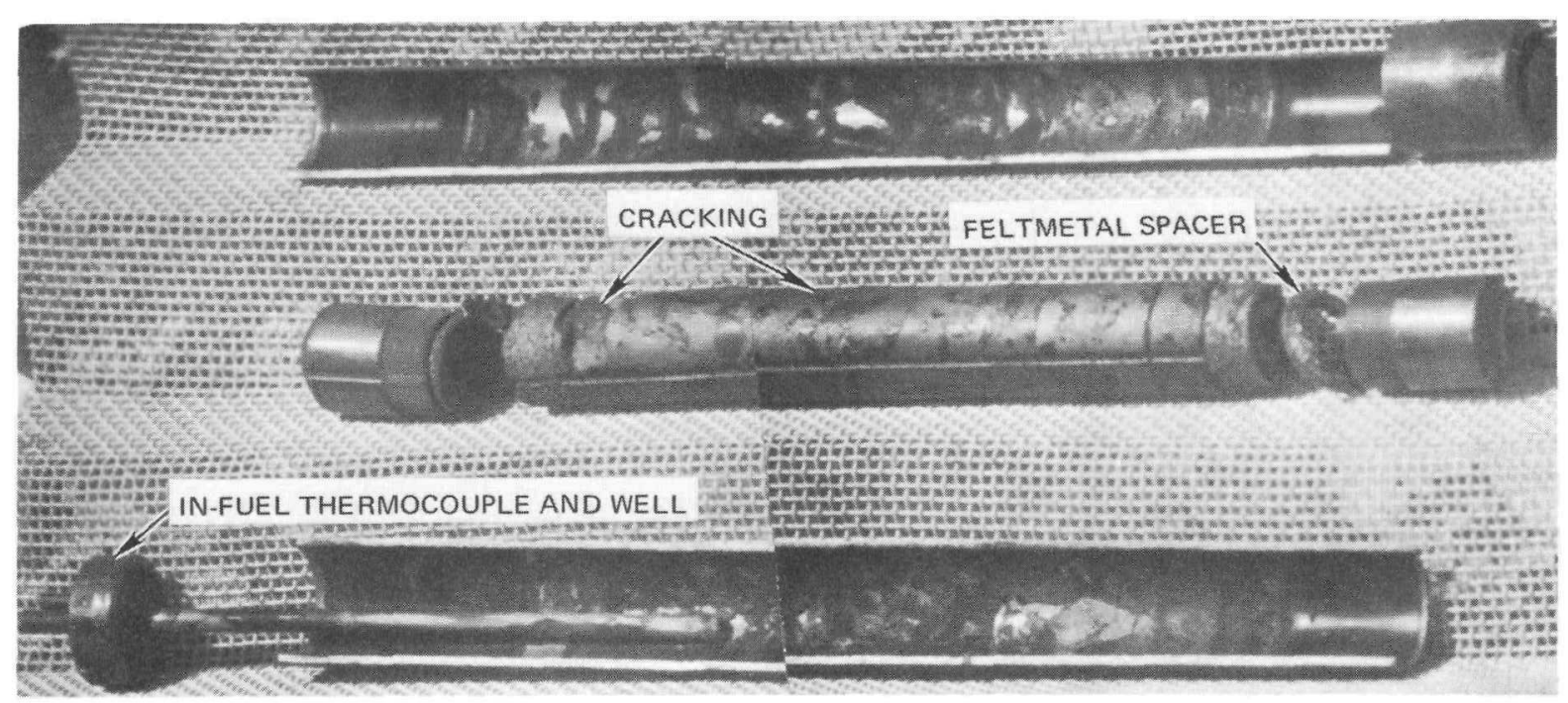

$120-427,-428$

b. After Disassembly

Figure 8. Specimen M-1

AI-AEC- 12963

35 


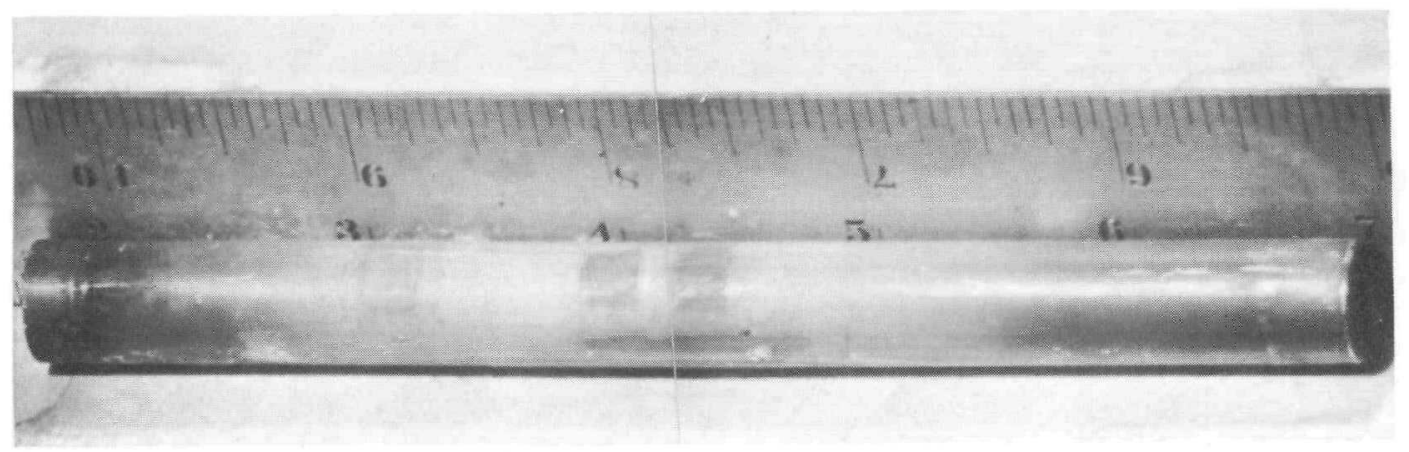

$120-175,-176$

a. Before Disassembly

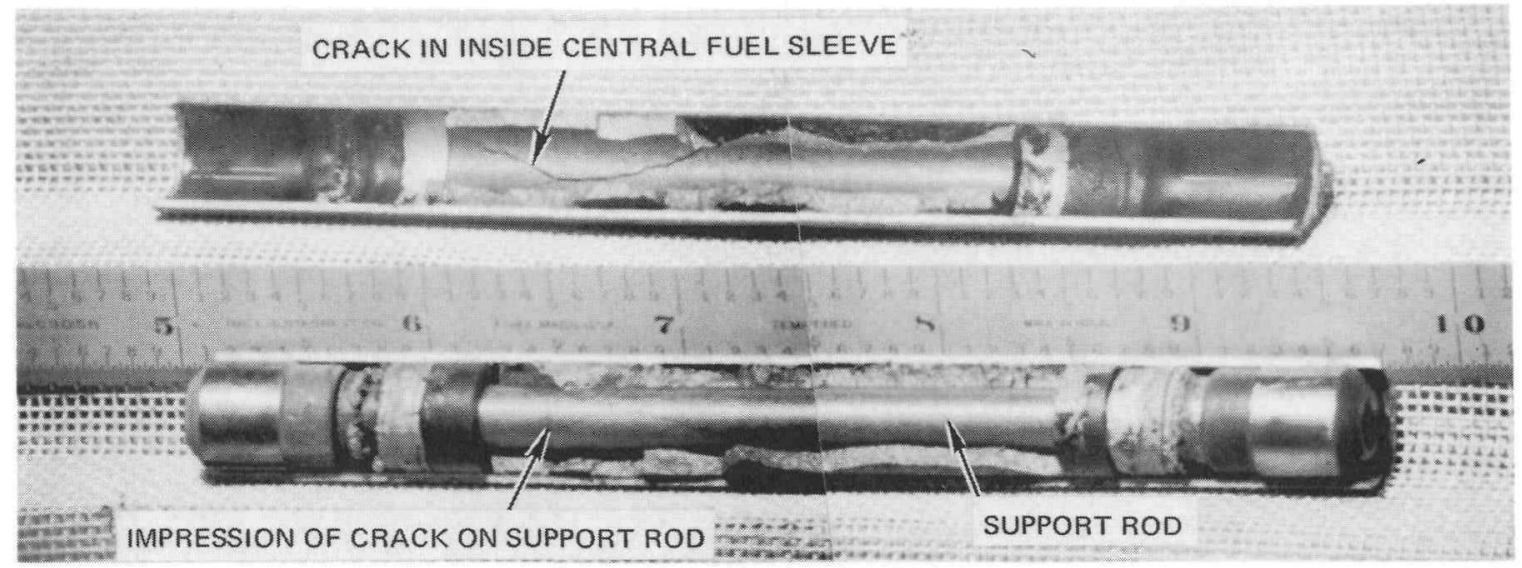

$120-353,-354$

b. After Disassembly

Figure 9. Specimen E-4 
respectively. The fuel sleeve surface was very rough on Specimens E-1, E-4, E-5, H-4, and H-5 [all of these specimens contained a high $\mathrm{H} / \mathrm{Z}_{\mathrm{r}}$ (1.85), and operated at 1300 and $1500^{\circ} \mathrm{F}$ respectively].

\section{FUEL MEASUREMENTS \\ 1. Dimensional Measurements}

The dimensional measurements were obtained with a profilometer measur ing device and/or dial indicator, with backup measurements using Vernier calipers. In some cases, the dimensional measurements were deduced from neutron radiographs. Profilometer diameter measurements could only be obtained on the unbroken fuel specimens.

The tabulated postirradiation vs pre-irradiation dimensional measurements, and the hydrogen-loss-corrected postirradiation vs the pre-irradiation measurements, are shown in Table 9; included in the measurements are the diameters taken on the support rod (normal U), directly under the center fuel sleeve.

Hydrogen redistribution and loss during irradiation causes fuel shrinkage, leading to density, diameter, and length changes, in addition to those caused by radiation-induced swelling. The correction for density values was taken directly from the plot of density vs hydrogen content. (6) The hydrogen analysis performed on the fuel specimens is described in a later section. Correction factors for the dimensions were calculated from the corrected densities in the following manner:

$(\% \Delta D / D)_{\text {hydrogen correction }}=(\% \Delta \rho / \rho)_{\text {hydrogen correction }} / 2.5$

$(\% \Delta L / L)_{\text {hydrogen correction }}=(\% \Delta \rho / \rho)_{\text {hydrogen correction }} / 5.0$

Addition of these corrections to the measured values yielded the corrected values shown in Table 10. The corrections were obtained from hydriding data that produced the ratio $(\% \Delta D / D) /(\% \Delta L / L)=2.0 .^{(3)}$

\section{Fuel Density Measurements}

The fuel specimen densities were obtained, using the liquid displacement technique, with monobromobenzene as the liquid medium. The accuracy of 
TABLE 9

FUEL DIMENSIONAL MEASUREMENTS

\begin{tabular}{|c|c|c|c|c|c|c|c|c|c|c|c|c|c|c|}
\hline \multirow{2}{*}{$\begin{array}{l}\text { Test } \\
\text { Specimen }\end{array}$} & \multirow{2}{*}{$\begin{array}{c}\text { Instual } \\
\text { Diametral } \\
\text { Gap } \\
\text { (1n) }\end{array}$} & \multirow{2}{*}{$\begin{array}{c}\text { Pre- } \\
\text { Irradiation } \\
\text { OD } \\
\text { (nn) }\end{array}$} & \multicolumn{4}{|c|}{$\begin{array}{c}\text { Diameter Increase } \\
\text { (in })\end{array}$} & \multirow{2}{*}{$\begin{array}{c}\text { Average } \\
\% \Delta \mathrm{D} / \mathrm{D} \\
\text { (hydrogen } \\
\text { cortected) }\end{array}$} & \multirow{2}{*}{$\begin{array}{c}\text { Initial } \\
\text { Axial } \\
\text { Gap } \\
\text { (1n) }\end{array}$} & \multirow{2}{*}{$\begin{array}{l}\text { Length } \\
\text { Increase } \\
\text { (in) }\end{array}$} & \multirow{2}{*}{$\begin{array}{c}\% \Delta L / L \\
\text { (hydrogen } \\
\text { corrected) }\end{array}$} & \multicolumn{3}{|c|}{$\begin{array}{l}\text { Average Support Rod Diameter } \\
\text { (1n })\end{array}$} & \multirow{2}{*}{$\begin{array}{l}\text { Support } \\
\text { Rod } \\
\text { Average } \\
\text { \% } \Delta D / D \\
\text { (hydrogen } \\
\text { corrected }\end{array}$} \\
\hline & & & Top & Center & Bottom & $\begin{array}{c}\text { Average } \\
\Delta \mathrm{D} \\
\text { (hydrogen } \\
\text { corrected) }\end{array}$ & & & & & $\begin{array}{c}\text { Pre- } \\
\text { Irradiation }\end{array}$ & $\begin{array}{c}\text { Post } \\
\text { Irradiation }\end{array}$ & $\Delta \mathrm{D}$ & \\
\hline $\mathrm{E} 1$ & $00084 \pm 00028$ & 04092 & 00081 & 00081 & 00076 & 00083 & 203 & 0080 & 0051 & 227 & 02502 & 02480 & -00022 & 08 \\
\hline$E-2^{*}$ & $00084 \pm 00016$ & 04092 & 00065 & 00069 & 00068 & 00069 & 169 & 0050 & 0.031 & 138 & 02501 & 02516 & 00015 & 16 \\
\hline $\mathrm{E} 3^{\circ}$ & $00088 \pm 00026$ & 04091 & 00066 & 00063 & - & 00065 & 159 & 0090 & 0025 & 111 & 02501 & 02518 & 00017 & 19 \\
\hline$E-4$ & $00082 \pm 00024$ & 04093 & - & - & - & $00097^{\dagger}$ & 237 & 0037 & 0001 & 04 & 02502 & 02460 & -00042 & 40 \\
\hline$E-5^{*}$ & $00084 \pm 00016$ & 04091 & 00073 & 00074 & 00072 & 00073 & 178 & 0088 & 0053 & 236 & 02503 & 02504 & 00001 & 02 \\
\hline $\mathrm{H}-1^{*}$ & $00162 \pm 00030$ & 04011 & 00121 & 00120 & 00120 & 00121 & 302 & 0129 & 0055 & 244 & 02500 & 02504 & 00004 & 20 \\
\hline $\mathrm{H}-2$ & $00164 \pm 00032$ & 04007 & 00162 & 00156 & 00165 & 00178 & 444 & 0044 & 0060 & 267 & 02502 & 02485 & -00017 & 06 \\
\hline $\mathrm{H}-3^{*}$ & $00168 \pm 00026$ & 04011 & 00138 & 00132 & 00134 & 00135 & 337 & 0075 & 0064 & 285 & 02501 & 02504 & 00003 & 07 \\
\hline $\mathrm{H}-4^{*}$ & $00168 \pm 00026$ & 04011 & 00106 & 00106 & 00104 & 00109 & 272 & 0054 & 0025 & 111 & 02502 & 02515 & 00013 & 20 \\
\hline $\mathrm{H}-5$ & $00168 \pm 00032$ & 04011 & 00117 & 00126 & 00146 & 00131 & 327 & 0028 & 0001 & 004 & 02502 & 02480 & -00022 & 18 \\
\hline$M-1$ & $00252 \pm 00016$ & 03921 & 00267 & 00260 & 00264 & 00284 & 724 & 0110 & $0099^{\S}$ & 440 & 02501 & 02450 & -00051 & 12 \\
\hline$M-2$ & $00258 \pm 00036$ & 03921 & 00270 & 00262 & 00250 & 00293 & 747 & 0076 & $0116^{\S}$ & 516 & 02501 & 02480 & -00021 & 23 \\
\hline$M-3$ & $00260 \pm 00038$ & 03921 & - & - & - & $00283^{\dagger}$ & 722 & 0093 & $0115^{\S}$ & 510 & 02500 & No meas & - & - \\
\hline$M-4^{*}$ & $00256 \pm 00036$ & 03921 & 00194 & 00202 & 00205 & 00206 & 525 & 0085 & 0083 & 369 & 02503 & 02515 & 00012 & 27 \\
\hline$M-5^{*}$ & $00258 \pm 00048$ & 03923 & 00211 & 00203 & 00193 & 00211 & 538 & 0043 & 0100 & 445 & 02502 & 02525 & 00023 & 31 \\
\hline$v-1$ & $00160 \pm 00024$ & 04012 & 00080 & 00102 & 00070 & 00117 & 292 & 0089 & $0051^{\S}$ & 227 & 02500 & 02490 & -00010 & 15 \\
\hline $\mathrm{V}-2$ & $00160 \pm 00022$ & 04011 & - & - & - & $00175^{\dagger}$ & 436 & 0076 & $0084^{\S}$ & 373 & 02501 & 02470 & 00031 & 27 \\
\hline
\end{tabular}


TABLE 10

HYDROGEN-CORRECTED FUEL DENSITY MEASUREMENTS

\begin{tabular}{|c|c|c|c|c|c|c|c|c|c|c|c|c|c|c|c|}
\hline \multirow{3}{*}{$\begin{array}{l}\text { Specimen } \\
\text { Number }\end{array}$} & \multicolumn{10}{|c|}{ Center Fuel Sleeve } & \multicolumn{5}{|c|}{ Support Rod } \\
\hline & \multirow{2}{*}{$\begin{array}{c}\text { Pre- } \\
\rho_{1} \\
(\mathrm{gm} / \mathrm{cc})\end{array}$} & \multicolumn{3}{|c|}{$\begin{array}{l}\text { Post }-\rho_{2} \\
(\mathrm{gm} / \mathrm{cc})\end{array}$} & \multicolumn{3}{|c|}{$\begin{array}{c}\Delta \rho \\
(\mathrm{gm} / \mathrm{cc})\end{array}$} & \multicolumn{3}{|c|}{$\begin{array}{c}\text { Volume Change } \\
\left(\Delta \rho / \rho_{2}\right) \\
(\%)\end{array}$} & \multirow{2}{*}{$\begin{array}{c}\text { Pre- } \\
\rho_{1} \\
(\mathrm{gm} / \mathrm{cc})\end{array}$} & \multirow{2}{*}{$\begin{array}{c}\text { Post- } \\
\mathrm{O}_{2} \\
\text { (gm/cc) }\end{array}$} & \multirow{2}{*}{$\begin{array}{c}\Delta \rho \\
(\mathrm{gm} / \mathrm{cc})\end{array}$} & \multirow{2}{*}{$\begin{array}{c}\text { Change } \\
\left(\Delta \rho / \rho_{1}\right) \\
(\%)\end{array}$} & \multirow{2}{*}{$\begin{array}{c}\text { Volume } \\
\text { Change } \\
\left(\Delta \rho / \rho_{2}\right) \\
(\%)\end{array}$} \\
\hline & & Top $*$ & Bulk & Bottom & Top & Bulk & Bottom & Top & Bulk & Bottom & & & & & \\
\hline$E-1$ & 6.024 & & 5.607 & & & 0.417 & & & 7.44 & & 6.059 & 6.019 & 0.040 & 0.66 & 0.66 \\
\hline$E-2$ & 6.048 & 5.802 & 5.777 & 5.759 & 0.246 & 0.271 & 0.289 & 4.23 & 4.69 & 5.01 & 6.077 & 5.990 & 0.087 & 1.43 & 1.45 \\
\hline$E-3$ & 6.046 & & 5.801 & & & 0.245 & & & 4.22 & & 6.080 & 5.974 & 0.106 & 1.74 & 1.77 \\
\hline$E-4$ & 6.028 & & 5.720 & & & 0.308 & & & 5.38 & & 6.062 & 6.057 & 0.005 & 0.08 & 0.08 \\
\hline$E-5$ & 6.052 & 5.679 & 5.674 & 5.734 & 0.373 & 0.378 & 0.318 & 6.56 & 6.66 & 5.54 & 6.060 & 6.046 & 0.014 & 0.23 & 0.23 \\
\hline $\mathrm{H}-\mathrm{l}$ & 6.047 & & 5.519 & & & 0.528 & & & 9.57 & & 6.076 & 6.009 & 0.067 & 1.10 & 1.11 \\
\hline $\mathrm{H}-2$ & 6.069 & & 5.359 & & & 0.710 & & & 13.25 & & 6.078 & 6.058 & 0.020 & 0.33 & 0.33 \\
\hline $\mathrm{H}-3$ & 6.071 & 5.564 & 5.516 & 5.555 & 0.507 & 0.555 & 0.516 & 9.11 & 10.06 & 9.28 & 6.083 & 6.069 & 0.014 & 0.23 & 0.23 \\
\hline $\mathrm{H}-5$ & 6.052 & 5.570 & 5.577 & 5.627 & 0.482 & 0.475 & 0.426 & 8.65 & 8.52 & 8.56 & 6.048 & 5.994 & 0.054 & 0.89 & 0.90 \\
\hline$M-1$ & 6.070 & & 4.751 & & & 1.319 & & & 27.76 & & 6.078 & 6.042 & 0.036 & 0.59 & 0.60 \\
\hline$M-2$ & 6.045 & & 4.902 & & & 1.143 & & & 23.32 & & 6.077 & 5.963 & 0.114 & 1.88 & 1.91 \\
\hline$M-3$ & 6.044 & & 4.812 & & & 1.232 & & & 25.60 & & 6.070 & 5.838 & 0.232 & 3.82 & 3.97 \\
\hline$M-4$ & 6.025 & 5.337 & 5.300 & 5.299 & 0.688 & 0.725 & 0.726 & 12.89 & 13.68 & 13.70 & 6.057 & 5.949 & 0.108 & 1.78 & 1.82 \\
\hline$M-5$ & 6.022 & 5.170 & 5.178 & 5.221 & 0.852 & 0.844 & 0.801 & 16.47 & 16.30 & 15.34 & 6.057 & 5.895 & 0.162 & 2.67 & 2.75 \\
\hline $\mathrm{V}-1$ & 6.060 & & 5.682 & & & 0.378 & & & 6.65 & & 6.076 & 6.012 & 0.064 & 1.05 & 1.06 \\
\hline$V-2$ & 6.047 & & 5.170 & & & 0.877 & & & 16.96 & & 6.074 & 6.074 & 0.000 & 0.00 & 0.00 \\
\hline
\end{tabular}

*"Top" indicates end of specimen toward capsule lead tube. 
TABLE 11

NAA 120-4 BURNUP ANALYSIS

\begin{tabular}{|c|c|c|c|c|c|c|}
\hline \multirow{3}{*}{$\begin{array}{l}\text { Element } \\
\text { Number }\end{array}$} & \multicolumn{3}{|c|}{$\begin{array}{c}\text { Burnup (metal at. \%) } \\
\text { Measured By }\end{array}$} & \multicolumn{3}{|c|}{$\begin{array}{c}\text { Gamma Scan Results } \\
(\mathrm{Zr} 95 \text { Photopeak) }\end{array}$} \\
\hline & & & & \multirow{2}{*}{$\begin{array}{c}\text { Peak } \\
\text { Gamma } \\
\text { Activity } \\
\text { (counts / } \\
5 \text { sec) }\end{array}$} & \multirow{2}{*}{$\begin{array}{c}\text { Average } \\
\text { Gamma } \\
\text { Activity } \\
\text { (counts } \\
4 \text { sec) }\end{array}$} & \multirow{2}{*}{$\begin{array}{c}\text { Peak-to- } \\
\text { Average } \\
\text { Activity } \\
\text { Ratio }\end{array}$} \\
\hline & $\begin{array}{c}\text { Mass } \\
\text { Spectrometry }\end{array}$ & Dosimeter & $\begin{array}{c}\text { Thermal } \\
\text { Calculation }\end{array}$ & & & \\
\hline E-1 & 0.83 & 0.87 & 1.03 & 5484 & 5324 & 1.03 \\
\hline$E-2$ & 0.82 & 0.81 & 1.03 & 5522 & 5321 & 1.04 \\
\hline$E-3$ & 0.83 & 0.79 & 1.03 & 5379 & 5256 & 1.02 \\
\hline$E-4$ & 0.86 & 0.85 & 1.03 & 6144 & 5865 & $1.05^{\dagger}$ \\
\hline$E-5$ & 0.85 & $*$ & 1.03 & 6240 & 6063 & 1.03 \\
\hline $\mathrm{H}-1$ & 0.83 & 0.75 & 0.96 & 5692 & 5403 & 1.05 \\
\hline $\mathrm{H}-2$ & 0.86 & 0.69 & 0.96 & 5551 & 5405 & $1.03^{\dagger}$ \\
\hline $\mathrm{H}-3$ & 0.84 & $*$ & 0.96 & 5414 & 5191 & 1.04 \\
\hline $\mathrm{H}-4$ & 0.87 & 0.89 & 0.96 & 5938 & 5761 & 1.03 \\
\hline $\mathrm{H}-5$ & 0.89 & 0.93 & 0.96 & 6553 & 6196 & $1.06^{\dagger}$ \\
\hline$M-1$ & 0.82 & 0.52 & 0.90 & 3188 & 3021 & $1.06^{\dagger}$ \\
\hline$M-2$ & 0.81 & 0.69 & 0.90 & 3278 & 2978 & $1.10^{\dagger}$ \\
\hline$M-3$ & 0.82 & 0.71 & 0.90 & 3483 & 3157 & $1.10^{\dagger}$ \\
\hline$M-4$ & 0.85 & 0.72 & 0.90 & 3305 & 3204 & 1.03 \\
\hline$M-5$ & 0.85 & 0.74 & 0.90 & 3349 & 3238 & 1.03 \\
\hline $\mathrm{V}-1$ & 0.82 & 1.00 & 0.89 & 3856 & 3756 & $1.04^{\dagger}$ \\
\hline$V-2$ & 0.85 & 0.82 & 0.82 & 3575 & 3423 & $1.04^{\dagger}$ \\
\hline
\end{tabular}

*Data not available

†Fuel specimen badly cracked and broken 
these measurements has been determined, from previous measurements, to be within $0.1 \%$ of the cited values. Table 10 shows the hydrogen-cor rected density measurements. The hydrogen-corrected densities are based on the relationship:

$$
(\Delta \rho / \rho)_{\text {swelling }}=(\Delta \rho / \rho)_{\text {measured }}+(\Delta \rho / \rho)_{\text {hydrogen correction }}
$$

The hydrogen correction was obtained from published curves of $\rho \mathrm{vs} H / \mathrm{Zr}$ ( ${ }^{(6)}$ The density measurements showed volume changes from 4.22 to $27.76 \%$, after correcting for hydrogen loss.

\section{Fuel Sampling}

Each specimen was sectioned across the center fuel sleeve and support rod, to provide samples for burnup determinations (center fuel sleeve only), hydrogen analysis, and metallographic examination. All of the samples were taken at approximately the same location, as shown in the following sketch, except when the broken and/or cracked condition of the fuel dictated the sample location.

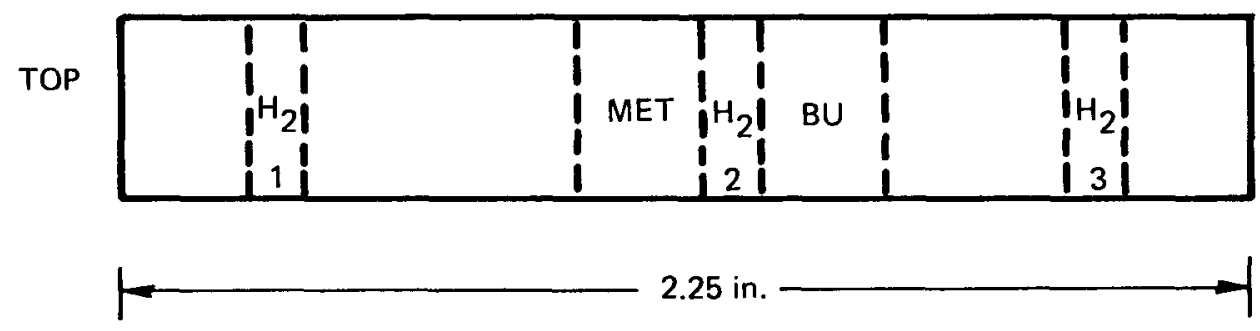

\section{Fuel Burnup Measurements}

A representative section of fuel from the middle portion of the center fuel sleeves was analyzed for uranium isotopic content by mass spectrometry. This analysis was used to calculate the metal atom percent burnup for the 17 fuel specimens from the NAA 120 Experiment. The dosimeter analysis for burnup was calculated from three foils ( $\mathrm{Ni}, \mathrm{Fe}$, and $\mathrm{Co}$ ) which formed a single dosimeter, banded to each of the 17 elements during the test.

The mass spectrographic and dosimeter burnup values are shown in Table 11, along with the value calculated from thermal data. As can be seen in Table 11, 
TABLE 12

NAA 120-4 HYDROGEN ANALYSIS ${ }^{*}$

\begin{tabular}{|c|c|c|c|c|c|c|c|c|c|c|}
\hline \multirow{3}{*}{$\begin{array}{l}\text { Specimen } \\
\text { Number }\end{array}$} & \multicolumn{5}{|c|}{ Center Sleeve } & \multicolumn{5}{|c|}{ Center $2-1 / 4$ in. Support Rod } \\
\hline & \multirow{2}{*}{$\begin{array}{l}\text { Pre- } \\
\mathrm{H} / \mathrm{Zr}_{\mathrm{r}}\end{array}$} & \multicolumn{3}{|c|}{ Post-H/Zr } & \multirow{2}{*}{$\begin{array}{c}\text { Average } \\
\mathrm{H} / \mathrm{Zr}\end{array}$} & \multirow{2}{*}{$\begin{array}{l}\text { Pre- } \\
\mathrm{H} / \mathrm{Zr}_{\mathrm{r}}\end{array}$} & \multicolumn{3}{|c|}{ Post-H/Zr } & \multirow{2}{*}{$\begin{array}{c}\text { Average } \\
\mathrm{H} / \mathrm{Zr}\end{array}$} \\
\hline & & Top & Center & Bottom & & & Top & Center & Bottom & \\
\hline$E-1$ & 1.85 & $1.77^{\dagger}$ & $1.75^{\dagger}$ & $1.71^{\dagger}$ & 1.74 & 1.85 & $1.77^{\dagger}$ & $1.75^{\dagger}$ & $1.71^{\dagger}$ & 1.74 \\
\hline$E-2$ & 1.72 & 1.74 & 1.74 & 1.72 & 1.73 & 1.69 & 1.65 & 1.67 & 1.70 & 1.67 \\
\hline$E-3$ & 1.72 & 1.72 & 1.68 & 1.72 & 1.71 & 1.71 & 1.66 & 1.65 & 1.67 & 1.66 \\
\hline E-4 & 1.86 & $\begin{array}{c}\text { Sample } \\
\text { lost }\end{array}$ & 1.82 & 1.81 & 1.81 & 1.85 & 1.78 & 1.78 & 1.77 & 1.78 \\
\hline $\mathrm{E}-5$ & 1.86 & 1.83 & 1.86 & 1.86 & 1.85 & 1.85 & 1.84 & 1.86 & 1.88 & 1.86 \\
\hline $\mathrm{H}-1$ & 1.71 & 1.66 & 1.66 & 1.67 & 1.66 & 1.70 & 1.51 & 1.53 & 1.57 & 1.54 \\
\hline $\mathrm{H}-2$ & 1.71 & 1.74 & 1.58 & 1.44 & 1.59 & 1.70 & 1.68 & 1.57 & 1.50 & 1.58 \\
\hline $\mathrm{H}-3$ & 1.70 & 1.69 & 1.70 & 1.70 & 1.70 & 1.71 & 1.62 & 1.63 & 1.63 & 1.63 \\
\hline $\mathrm{H}-4$ & 1.85 & 1.76 & 1.74 & 1.72 & 1.74 & 1.85 & 1.68 & 1.68 & 1.69 & 1.68 \\
\hline $\mathrm{H}-5$ & 1.85 & 1.70 & 1.76 & $1.91^{\dagger}$ & 1.79 & 1.83 & 1.68 & 1.72 & $1.91^{\dagger}$ & 1.77 \\
\hline$M-1$ & 1.71 & $1.58^{\dagger}$ & $1.30^{\dagger}$ & 1.60 & 1.49 & 1.70 & $1.58^{\dagger}$ & $1.30^{\dagger}$ & 1.34 & 1.41 \\
\hline$M-2$ & 1.72 & 1.62 & 1.59 & $1.12^{\dagger}$ & 1.44 & 1.71 & 1.54 & 1.52 & $1.12^{\dagger}$ & 1.39 \\
\hline$M-3$ & 1.70 & 1.24 & $1.63^{\dagger}$ & $0.90^{\dagger}$ & 1.26 & 1.71 & 0.99 & $1.63^{\dagger}$ & $0.90^{\dagger}$ & 1.17 \\
\hline$M-4$ & 1.85 & 1.69 & 1.69 & 1.69 & 1.69 & 1.86 & 1.52 & 1.66 & 1.64 & 1.61 \\
\hline$M-5$ & 1.84 & 1.71 & 1.72 & 1.70 & 1.71 & 1.85 & 1.66 & 1.66 & 1.65 & 1.66 \\
\hline $\mathrm{V}-1$ & 1.71 & $1.51^{\dagger}$ & 1.49 & $1.47^{\dagger}$ & 1.49 & 1.72 & $1.51^{\dagger}$ & 1.46 & $1.47^{\dagger}$ & 1.48 \\
\hline$V-2$ & 1.71 & $1.63^{\dagger}$ & $1.61^{\dagger}$ & $1.61^{\dagger}$ & 1.62 & 1.71 & $1.63^{\dagger}$ & $1.61^{\dagger}$ & $1.61^{\dagger}$ & 1.62 \\
\hline
\end{tabular}

*Accuracy $\pm 0.02 \mathrm{H} / \mathrm{Zr}$

†Support rod and fuel sleeve analyzed together 
the burnup analyses failed to agree with each other. In past experiments of this nature, the analysis by mass spectrometry was considered more reliable, because it has the least degree of uncertainty. The mass spectrometry data on past experiments would tend to follow the gamma activity profile (also shown in Table 11) within $\sim 10 \%$. However, in the case of the mass spectrometry burnup for the NAA 120 Experiment, it was found, after normalizing the data, that correlation with the gamma activity existed only in the $E$ and $H$ elements. The reason for the disagreement in the data for the $M$ and $V$ elements is probably related to different thickness of the fuel sleeves and to fuel condition.

\section{Hydrogen Measurements}

Samples for hydrogen analysis were obtained as 1/8-in. thick wafers, across both the fuel sleeve and support rod. Three samples from each specimen were analyzed for hydrogen content, after being cleaned in ethyl alcohol and weighed.

The hydrogen analysis was performed at the Atomics International Hot Laboratory (AIHL), using a vacuum extraction analysis unit which involves induction heating the fuel samples in a vacuum at temperatures high enough to release the hydrogen $\left(\sim 2200^{\circ} \mathrm{F}\right)$. The released gas is collected in a calibrated volume, and the pressure of the hydrogen is measured. The accuracy associated with this type of measurement is considered to be \pm 0.02 wt $\%$ of that measured.

A summary of the hydrogen analyses is presented in Table 12. Some of the specimens showed steep hydrogen gradients, over the length of the specimens; in general, most of the support rods showed a slightly lower hydrogen content than the fuel sleeves.

\section{FUEL METALLOGRAPHIC EXAMINATION}

Metallographic samples of the fuel were taken from each of the fuel specimens, to determine what structural changes had occurred.

The samples were mounted with Hysol C-9 epoxy resin, and were ground on 240-,320-, and 600-grit silicon carbide paper, using water as a lubricant. Attack polishing was performed on the fuel samples, using rayon cloth with alternate application of a Linde-B slurry, a $\mathrm{HNO}_{3}-\mathrm{HF}-\mathrm{H}_{2} \mathrm{O}$ mixture, and a $10 \%$ oxalic acid solution. Electro-etching was performed in a $10 \%$ oxalic acid solution at 20 to 300-v dc. Many of the samples were reground, repolished, and etched again to confirm observations. 
TABLE 13

FILAR EYE DIAMETER FUEL MEASUREMENTS vs PRE-IRRADIATION FUEL MEASUREMENTS

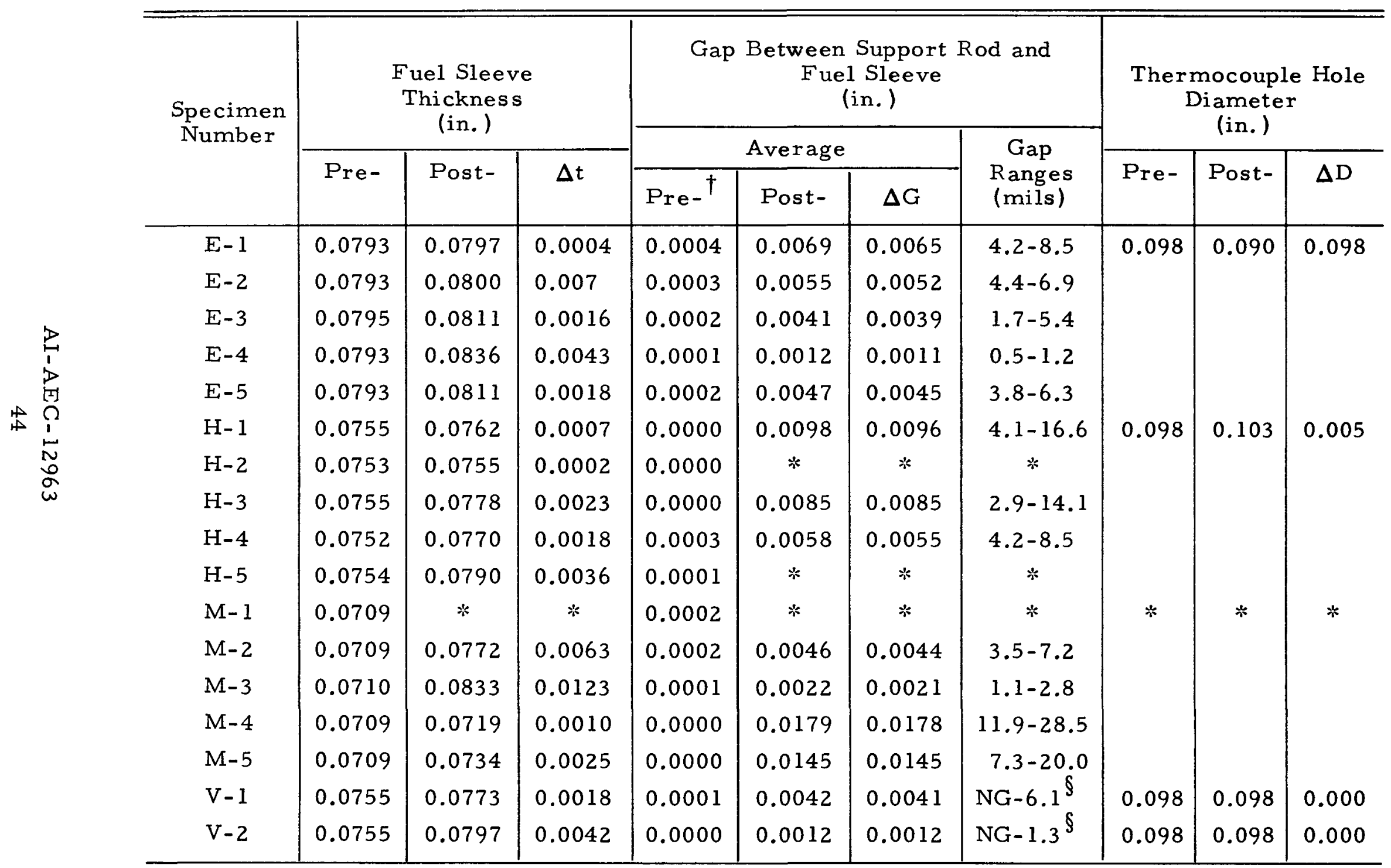

*Data not valid or not taken, due to fuel condition

$\dagger$ Pre-Gap measurements, calculated from published pre-irradiation data package ${ }^{(2)}$

$\$ N G$ - No gap 
It should be stressed that all postirradiation metallographic observations were based on phases and structures existing at room temperature, and at the final hydrogen level. During elevated-temperature reactor operations, and before loss of major amounts of hydrogen, completely different phases were present, as can easily be seen by consulting the zirconium-hydrogen phase diagram (Figure 1). Filar eye measurements on the fuel sleeve thickness, gap between sleeve and support rod, and thermocouple holes were also obtained from the metallographic samples. These data are shown in Table 13. Steep hydrogen gradients were observed in a number of cases, particularly in Specimens $M-1$, $\mathrm{M}-2$, and $\mathrm{M}-3\left(1600^{\circ} \mathrm{F}\right.$ specimens).

Earlier observation on Specimen H-5 indicated that this specimen was probably in two phases. The upper half of the specimen surface was smooth, while the lower half was rough, pitted, and broken. The hydrogen analysis supported this; the analysis showed $1.70 \mathrm{H} / \mathrm{Zr}_{\mathbf{r}}(\delta)$ at the top, and a $1.91 \mathrm{H} / \mathrm{Zr}(\epsilon)$ at the bottom of the specimen. The hydrogen analysis also indicated that Specimen V-2 was not in $\beta$ phase at the end of the test; however, the general appearance and condition of the fuel structure suggests $\beta$-phase operation. ${ }^{(22)}$ The NAA 120 fuel structure evaluation is tabulated in Table 14. Typical photomicrographs of the fuel sleeves, obtained during the metallographic examination, are shown in Figure 10. 
TABLE 14

NAA-120-4 FUEL MICROSTRUCTURE EVALUATION

(Metallographic and Hydrogen Analyzer)

\begin{tabular}{|c|c|c|c|c|c|c|c|c|c|c|c|}
\hline \multirow{3}{*}{$\begin{array}{l}\text { Specimen } \\
\text { Number }\end{array}$} & \multicolumn{11}{|c|}{ Central Fut 1 Sleeve and Adjacent Support Rod } \\
\hline & \multicolumn{3}{|c|}{ Phases Present } & \multicolumn{3}{|c|}{ Sample Structure Condition } & \multicolumn{3}{|c|}{ Postirradiation $\mathrm{H} / \mathrm{Z}_{\mathrm{r}}$} & \multicolumn{2}{|c|}{$\begin{array}{c}\text { Ind1cated } \beta \text {-Phase } \\
\text { Operation }\end{array}$} \\
\hline & Top & Center & Bottom & Porosity & Voids & Cracks & Top & Center & Bottom & $\begin{array}{l}\text { Hydrogen } \\
\text { Analysis }\end{array}$ & $\begin{array}{r}\text { Metallo- } \\
\text { graphic }\end{array}$ \\
\hline $\begin{array}{l}\text { E-1 sle eve } \\
\text { F-1 support }\end{array}$ & & $\epsilon\langle\delta+\epsilon\rangle$ & & $\begin{array}{l}\text { Yes } \\
\text { No }\end{array}$ & $\begin{array}{l}\text { No } \\
\text { No }\end{array}$ & $\begin{array}{l}\text { Micro-edge } \\
\text { No }\end{array}$ & $\begin{array}{ll}177^{n * x} \\
177^{* x}\end{array}$ & $\begin{array}{ll}1 & 75^{\circ} \\
1 & 75^{\circ}\end{array}$ & $\begin{array}{lll}1 & 7 & 1 \\
1 & 71^{*}\end{array}$ & $\begin{array}{l}\text { No } \\
\text { No }\end{array}$ & $\begin{array}{l}\text { No } \\
\text { No }\end{array}$ \\
\hline $\begin{array}{l}\text { E-2 sleeve } \\
\text { E-2 support }\end{array}$ & $\epsilon^{\dagger}$ & $\begin{array}{l}\delta+\epsilon \\
\delta(\delta+\epsilon)\end{array}$ & & $\begin{array}{l}\text { No } \\
\text { No }\end{array}$ & $\begin{array}{l}\text { Yes } \\
\text { No }\end{array}$ & $\begin{array}{l}\text { No } \\
\text { No }\end{array}$ & $\begin{array}{ll}1 & 74 \\
1 & 65\end{array}$ & $\begin{array}{ll}1 & 74 \\
1 & 67\end{array}$ & $\begin{array}{l}172 \\
170\end{array}$ & $\begin{array}{l}\text { No } \\
\text { No }\end{array}$ & $\begin{array}{l}\text { No } \\
\text { No }\end{array}$ \\
\hline $\begin{array}{l}\text { E-3 sleeve } \\
E-3 \text { support }\end{array}$ & & $\begin{array}{l}\delta+\epsilon \\
\delta+\epsilon\end{array}$ & & $\begin{array}{l}\text { No } \\
\text { No }\end{array}$ & $\begin{array}{l}\text { No } \\
\text { No }\end{array}$ & $\begin{array}{l}\text { Micro-edge } \\
\text { Micro-edge }\end{array}$ & $\begin{array}{l}172 \\
166\end{array}$ & $\begin{array}{ll}1 & 68 \\
1 & 65\end{array}$ & $\begin{array}{ll}1 & 72 \\
1 & 67\end{array}$ & $\begin{array}{l}\text { No } \\
\text { No }\end{array}$ & $\begin{array}{l}\text { No } \\
\text { No }\end{array}$ \\
\hline E-4 sleeve & & $\epsilon(\delta+\epsilon)$ & & Yes & Yes & Yes & $\begin{array}{l}\text { Sample } \\
\text { lost }\end{array}$ & 182 & 181 & No & No \\
\hline E-4 support & & $\delta+\epsilon$ & & No & Yes & $\mathrm{M}_{1} \mathrm{cro-}$ & 178 & 178 & 177 & No & No \\
\hline $\begin{array}{l}\text { E-5 s leeve } \\
\text { E-5 support }\end{array}$ & & $\begin{array}{l}\epsilon(\delta+\epsilon) \\
\epsilon\end{array}$ & & $\begin{array}{l}\text { Yes } \\
\text { No }\end{array}$ & $\begin{array}{l}\text { No } \\
\text { No }\end{array}$ & $\begin{array}{l}\text { Micro- } \\
\text { No }\end{array}$ & $\begin{array}{ll}1 & 83 \\
1 & 84\end{array}$ & $\begin{array}{ll}1 & 86 \\
1 & 86\end{array}$ & $\begin{array}{ll}1 & 86 \\
1 & 88\end{array}$ & $\begin{array}{l}\text { No } \\
\text { No }\end{array}$ & $\begin{array}{l}\text { No } \\
\text { No }\end{array}$ \\
\hline $\begin{array}{l}\text { H-l sleeve } \\
H-1 \text { support }\end{array}$ & & $\begin{array}{l}\delta+\epsilon \\
\delta+\gamma^{\prime}\end{array}$ & & $\begin{array}{l}\text { Yes } \\
\text { Yes }\end{array}$ & $\begin{array}{l}\text { No } \\
\text { No }\end{array}$ & $\begin{array}{l}\text { Micro-edge } \\
\text { Micro-edge }\end{array}$ & $\begin{array}{ll}1 & 66 \\
1 & 51\end{array}$ & $\begin{array}{ll}1 & 66 \\
1 & 53\end{array}$ & $\begin{array}{ll}1 & 67 \\
1 & 57\end{array}$ & $\begin{array}{l}\text { No } \\
\text { Yes }\end{array}$ & $\begin{array}{l}\text { No } \\
\text { Yes }\end{array}$ \\
\hline $\begin{array}{l}\text { H-2 sleeve } \\
\text { H-2 support }\end{array}$ & & $\begin{array}{l}\delta+\gamma^{\prime} \\
\delta+\gamma^{\prime}\end{array}$ & $\begin{array}{l}\delta+\gamma^{\prime} \\
\delta+\gamma^{\prime}\end{array}$ & $\begin{array}{l}\text { Yes } \\
\text { Yes }\end{array}$ & $\begin{array}{l}\text { No } \\
\text { No }\end{array}$ & $\begin{array}{l}\text { Yes } \\
\text { Mucro-edge }\end{array}$ & $\begin{array}{ll}1 & 74 \\
1 & 68\end{array}$ & $\begin{array}{ll}1 & 58 \\
1 & 57\end{array}$ & $\begin{array}{ll}144 \\
1 & 50\end{array}$ & $\begin{array}{l}\text { Yes } \\
\text { Yes }\end{array}$ & $\begin{array}{l}\text { Yes } \\
\text { Yes }\end{array}$ \\
\hline $\begin{array}{l}\text { H-3 sleeve } \\
\text { H-3 support }\end{array}$ & & $\begin{array}{l}\delta+\epsilon \\
\delta\end{array}$ & & $\begin{array}{l}\text { Yes } \\
\text { Yes }\end{array}$ & $\begin{array}{l}\text { Yes } \\
\text { Yes }\end{array}$ & $\begin{array}{l}\text { Micro-edge } \\
\text { Micro-edge }\end{array}$ & $\begin{array}{ll}1 & 69 \\
1 & 62\end{array}$ & $\begin{array}{ll}1 & 70 \\
1 & 63\end{array}$ & $\begin{array}{ll}1 & 70 \\
1 & 63\end{array}$ & $\begin{array}{l}\text { No } \\
\text { No }\end{array}$ & $\begin{array}{l}\text { No } \\
\text { No }\end{array}$ \\
\hline $\begin{array}{l}\text { H-4 sleeve } \\
\text { H-4 support }\end{array}$ & & $\begin{array}{l}\delta+\epsilon \\
\delta+\epsilon\end{array}$ & & $\begin{array}{l}\text { No } \\
\text { No }\end{array}$ & $\begin{array}{l}\text { No } \\
\text { No }\end{array}$ & $\begin{array}{l}\text { Micro- } \\
\text { No }\end{array}$ & $\begin{array}{ll}176 \\
168\end{array}$ & $\begin{array}{ll}1 & 74 \\
1 & 68\end{array}$ & $\begin{array}{l}172 \\
160\end{array}$ & $\begin{array}{l}\text { No } \\
\text { No }\end{array}$ & $\begin{array}{l}\text { No } \\
\text { No }\end{array}$ \\
\hline $\begin{array}{l}\text { H-5 sleeve } \\
\text { H-5 support }\end{array}$ & $\delta, \epsilon$ traces & $\delta+\epsilon$ & $\delta+\epsilon$ & $\begin{array}{l}\text { Yes } \\
\text { Yes }\end{array}$ & $\begin{array}{l}\text { No } \\
\text { No }\end{array}$ & $\begin{array}{l}\text { Yes } \\
\text { Micro- }\end{array}$ & $\begin{array}{ll}1 & 70 \\
1 & 68\end{array}$ & $\begin{array}{ll}1 & 76 \\
1 & 72\end{array}$ & $\begin{array}{ll}191 \\
191\end{array}$ & $\begin{array}{l}\text { No } \\
\text { No }\end{array}$ & $\begin{array}{l}\text { No } \\
\text { No }\end{array}$ \\
\hline $\begin{array}{l}\text { M-l sleeve } \\
\text { M-1 support }\end{array}$ & & $\begin{array}{l}\delta+\gamma^{\prime} \\
\delta+\gamma^{\prime}\end{array}$ & & $\begin{array}{l}\text { Yes } \\
\text { Yes }\end{array}$ & $\begin{array}{l}\text { Yes } \\
\text { Yes }\end{array}$ & $\begin{array}{l}\text { Yes } \\
\text { Micro- }\end{array}$ & $\begin{array}{ll}1 & 58^{\prime \prime} \\
1 & 58^{\prime *}\end{array}$ & $\begin{array}{ll}130^{* n} \\
130^{*-x}\end{array}$ & $\begin{array}{l}160 \\
134\end{array}$ & $\begin{array}{l}\text { Yes } \\
\text { Yes }\end{array}$ & $\begin{array}{l}\text { Yes } t \dagger \\
\text { Yes }\end{array}$ \\
\hline $\begin{array}{l}\text { M-2 sleeve } \\
\text { M-2 support }\end{array}$ & & $\begin{array}{l}\delta+\gamma^{\prime} \\
\delta+\gamma^{\prime}\end{array}$ & $\begin{array}{l}\delta+\gamma^{\prime} \\
\delta+\gamma^{\prime}\end{array}$ & $\begin{array}{l}\text { Yes } \\
\text { Yes }\end{array}$ & $\begin{array}{l}\text { Yes } \\
\text { Yes }\end{array}$ & $\begin{array}{l}\text { Yes } \\
\text { Micro- }\end{array}$ & $\begin{array}{l}162 \\
154\end{array}$ & $\begin{array}{ll}1 & 59 \\
1 & 52\end{array}$ & $\begin{array}{ll}1 & 12^{* * *} \\
1 & 12\end{array}$ & $\begin{array}{l}\text { Yes } \\
\text { Yes }\end{array}$ & $\begin{array}{l}\text { Yes } t \dagger \\
\text { Yes }\end{array}$ \\
\hline $\begin{array}{l}\text { M-3 sleeve } \\
\text { M-3 support }\end{array}$ & & $\begin{array}{l}6+\gamma^{\prime} \\
6+\gamma^{\prime}\end{array}$ & & $\begin{array}{l}\text { Yes } \\
\text { Yes }\end{array}$ & $\begin{array}{l}\text { Yes } \\
\text { Yes }\end{array}$ & $\begin{array}{l}\text { Yes } \\
\text { Micro }\end{array}$ & $\begin{array}{ll}1 & 24 \\
0 & 99\end{array}$ & $\begin{array}{l}163^{* *} \\
163 *\end{array}$ & $\begin{array}{ll}0 & 90 \\
0 & 90^{* * *}\end{array}$ & $\begin{array}{l}\text { Yes } \\
\text { Yes }\end{array}$ & $\begin{array}{l}\text { Yes }{ }^{\dagger \dagger} \\
\text { Yes }\end{array}$ \\
\hline $\begin{array}{l}\text { M-4 sleeve } \\
\text { M-4 support }\end{array}$ & & $\begin{array}{l}\delta+\epsilon \\
\delta\end{array}$ & & $\begin{array}{l}\text { Yes } \\
\text { Yes }\end{array}$ & $\begin{array}{l}\text { No } \\
\text { No }\end{array}$ & $\begin{array}{l}\text { Micro-edge } \\
\text { Micro-edge }\end{array}$ & $\begin{array}{ll}1 & 69 \\
1 & 52\end{array}$ & $\begin{array}{ll}1 & 69 \\
1 & 66\end{array}$ & $\begin{array}{l}169 \\
164\end{array}$ & $\begin{array}{l}\text { No } \\
\text { Yes }\end{array}$ & $\begin{array}{l}\text { No } \\
\text { No }\end{array}$ \\
\hline $\begin{array}{l}\text { M-5 sleeve } \\
\text { M-5 support }\end{array}$ & & $\begin{array}{l}\delta \\
\delta\end{array}+\epsilon$ & & $\begin{array}{l}\text { No } \\
\text { No }\end{array}$ & $\begin{array}{l}\text { No } \\
\text { No }\end{array}$ & $\begin{array}{l}\text { No } \\
\text { No }\end{array}$ & $\begin{array}{ll}1 & 71 \\
1 & 66\end{array}$ & $\begin{array}{ll}1 & 72 \\
1 & 66\end{array}$ & $\begin{array}{ll}1 & 70 \\
1 & 65\end{array}$ & $\begin{array}{l}\text { No } \\
\text { No }\end{array}$ & $\begin{array}{l}\text { No } \\
\text { No }\end{array}$ \\
\hline $\begin{array}{l}V-1 \text { sleeve } \\
V-1 \text { support }\end{array}$ & & $\begin{array}{l}5+\gamma^{\prime} \\
5+\gamma^{\prime}\end{array}$ & & $\begin{array}{l}\text { Yes } \\
\text { Yes }\end{array}$ & $\begin{array}{l}\text { Yes } \\
\text { Yes }\end{array}$ & $\begin{array}{l}\text { Yes } \\
\text { Micro- }\end{array}$ & $\begin{array}{ll}1 & 51 \\
1 & 51\end{array}$ & $\begin{array}{l}149 \\
146\end{array}$ & $\begin{array}{l}147^{\cdots *} \\
147^{w}\end{array}$ & $\begin{array}{l}\text { Yes } \\
\text { Yes }\end{array}$ & $\begin{array}{l}\text { Yes } \\
\text { Yes }\end{array}$ \\
\hline $\begin{array}{l}V-2 \text { sleeve } \\
V-2 \text { support }\end{array}$ & & $\begin{array}{l}\delta \\
\delta\end{array}$ & & $\begin{array}{l}\text { Yes } \\
\text { Yes }\end{array}$ & $\begin{array}{l}\text { Yes } \\
\text { Yes }\end{array}$ & $\begin{array}{l}\text { Yes } \\
\text { Yes }\end{array}$ & $\begin{array}{ll}163^{* *} \\
163 *\end{array}$ & $\begin{array}{lll}1 & 6 & 1 * \cdots \\
1 & 6 & 1 * 4\end{array}$ & $\begin{array}{lll}1 & 61^{* * *} \\
1 & 61^{* *}\end{array}$ & $\begin{array}{l}\text { No } \\
\text { No }\end{array}$ & $\begin{array}{l}\text { Yes }{ }^{\dagger \dagger} \\
\text { Yest† }\end{array}$ \\
\hline
\end{tabular}

AI-A EC- 12963 

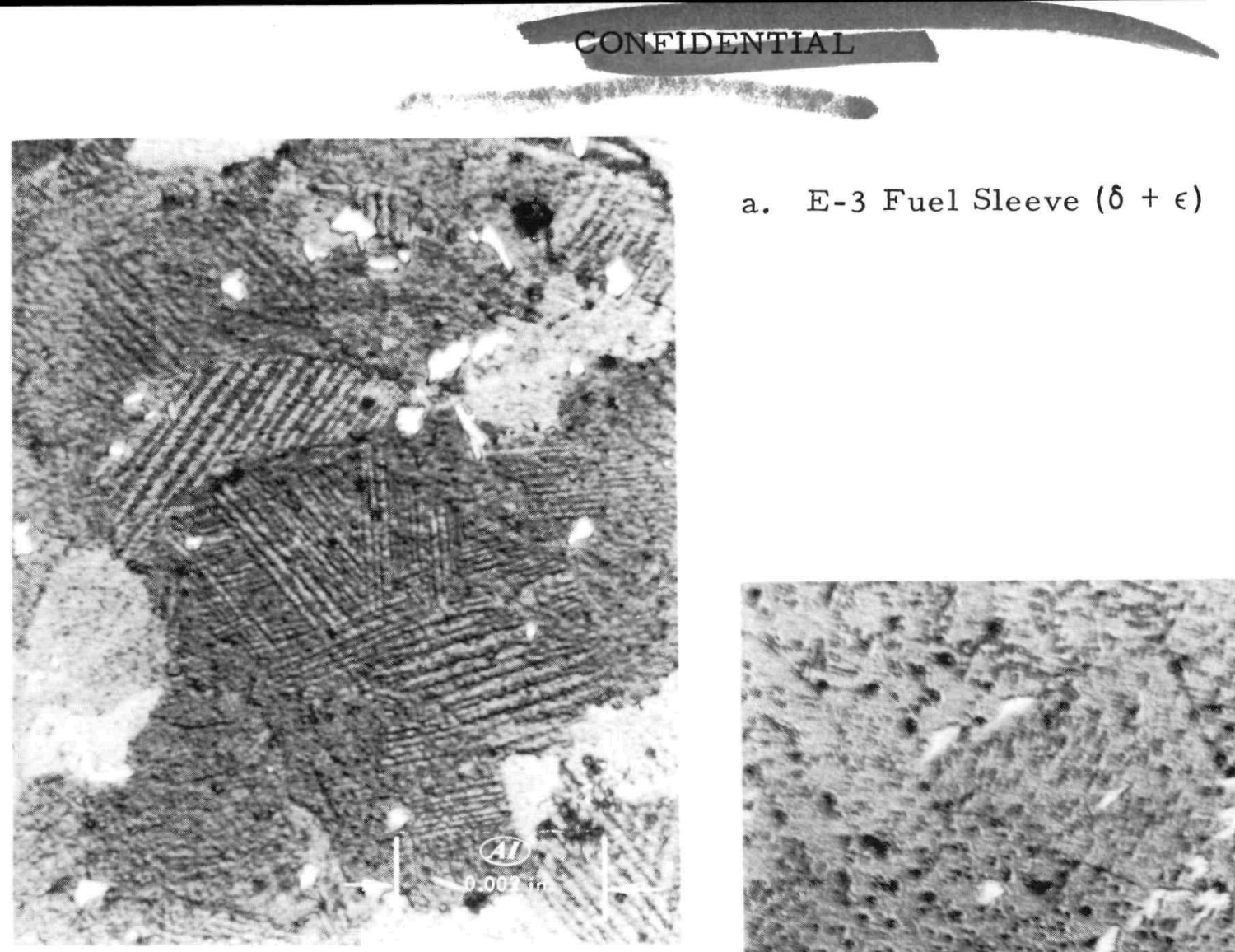

a. E-3 Fuel Sleeve $(\delta+\epsilon)$

$120-10-2$
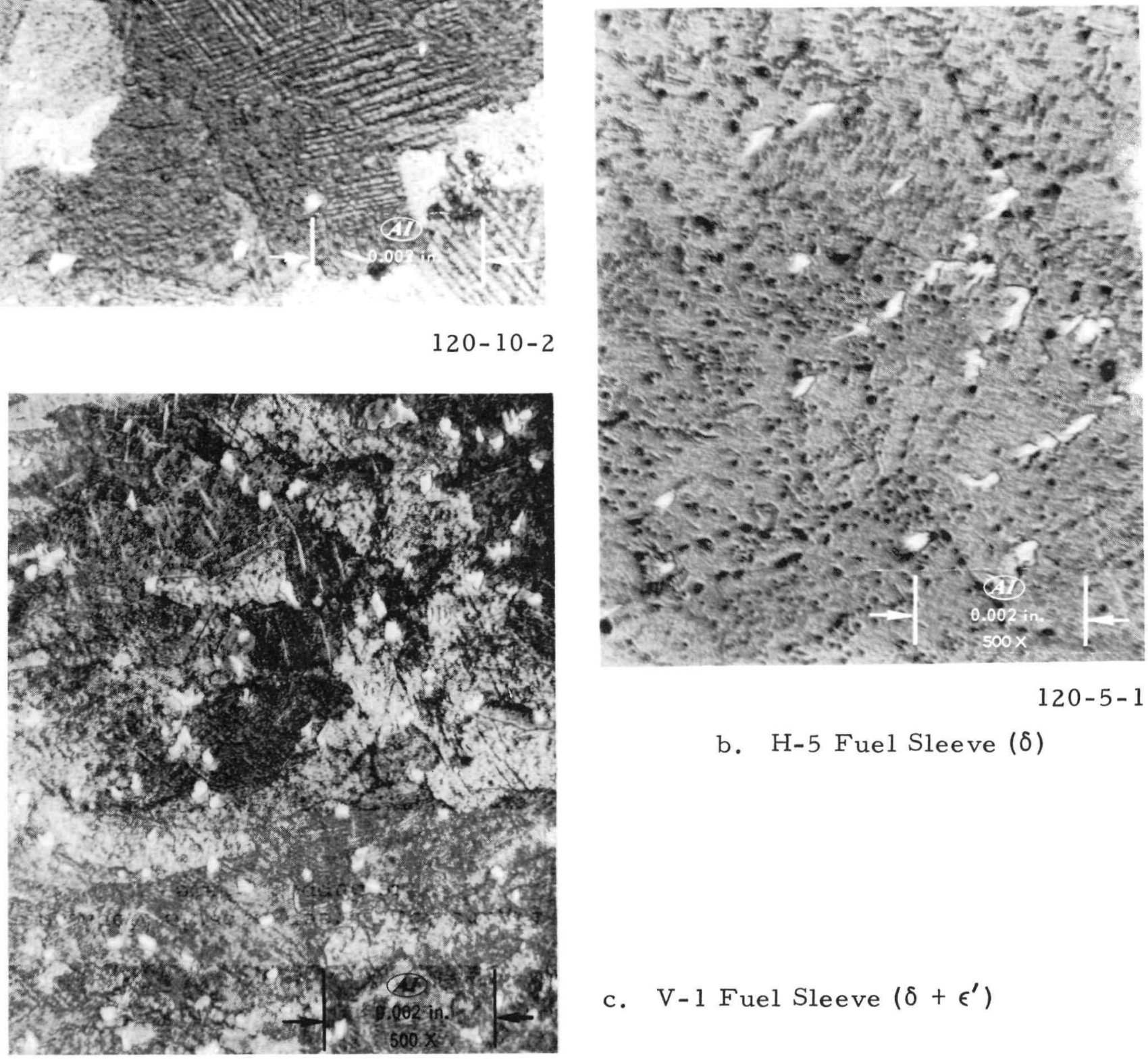

$120-5-1$

b. H-5 Fuel Sleeve ( $\delta)$

c. V-1 Fuel Sleeve $\left(\delta+\epsilon^{\prime}\right)$

121-6-1

Figure 10. Typical Photomicrographs of the NAA 120-4 Fuel (Figure Unclassified)

AI-AEC- 12963

47 


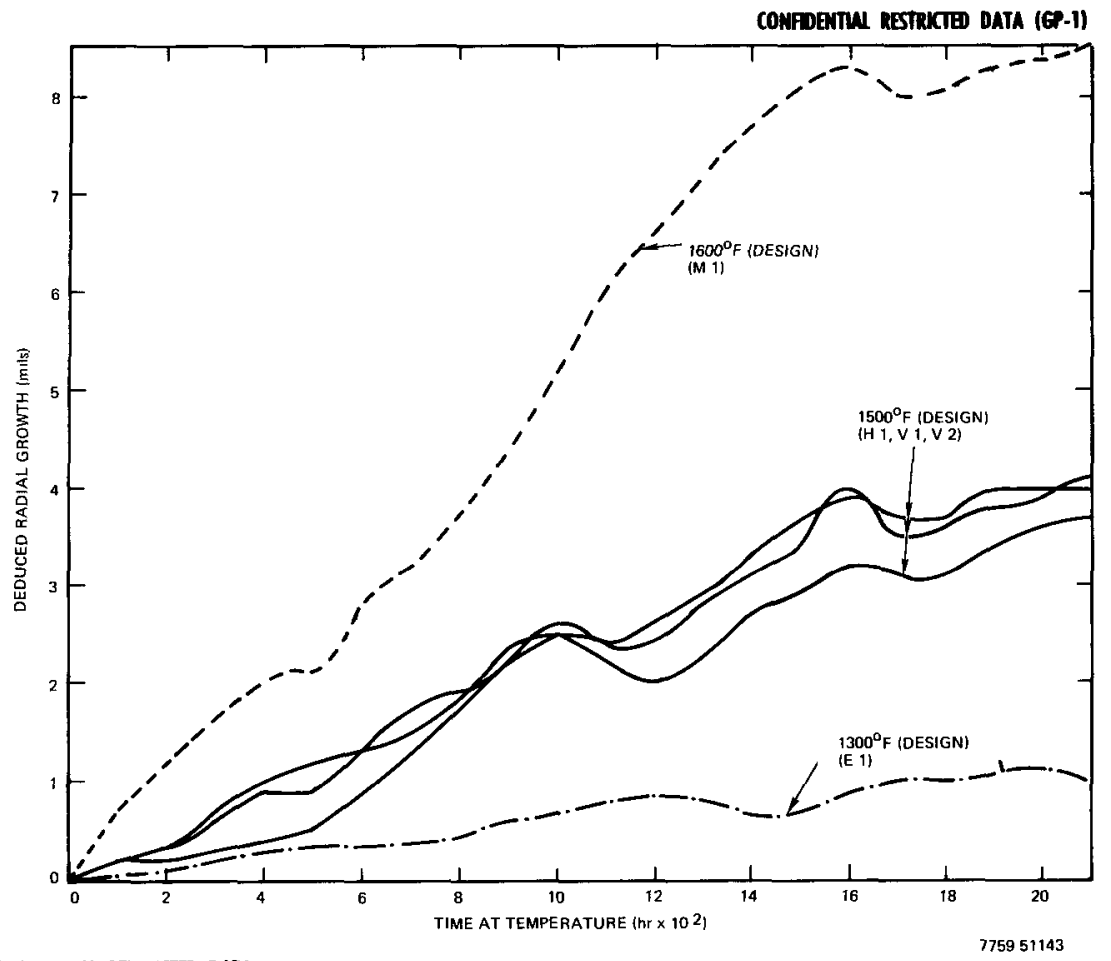

Figure 11. Radial Fuel Growth vs Time (Based on fuel-cladding $\Delta T$ )

CONFIDETLW RETRKCTED DATA

Figure 12. Volume Increase vs Burnup for the $1300^{\circ} \mathrm{F}$ Subcapsules (Based on fuel-cladding $\Delta \mathrm{T}$ )

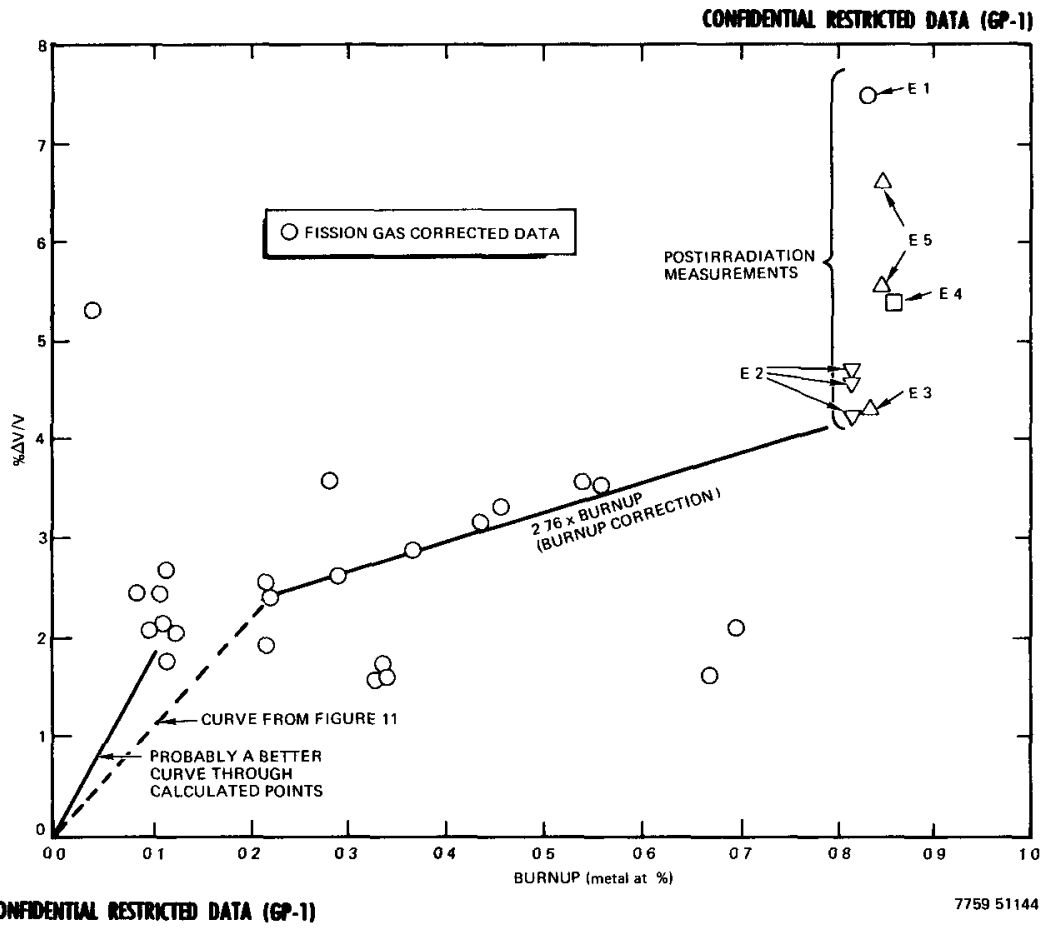

AI-AEC- 12963 


\section{DISCUSSION}

The temperature history of the NAA $120-4$ is of particular significance to the swelling model for $\mathrm{U}-\mathrm{ZrH}_{\mathrm{x}}$ fuel material. (23) Briefly, the present model, based on burnup and temperature, is represented by the following relationship:

$$
\begin{gathered}
\% \frac{\Delta \mathrm{V}}{\mathrm{V}}=\alpha \mathrm{B}+\beta \exp (-\lambda / \mathrm{T}) \\
\% \frac{\Delta \mathrm{V}}{\mathrm{V}}=\% \text { Volumetric fuel growth } \\
\left(\% \frac{\Delta \mathrm{V}}{\mathrm{V}}=2 \frac{\Delta \mathrm{D}}{\mathrm{D}}+\frac{\Delta \mathrm{L})}{\mathrm{L}}\right) \\
\mathrm{B}=\text { metal at. } \% \text { burnup } \\
\mathrm{T}=\text { bulk average fuel temperature }\left({ }^{\circ} \mathrm{F}\right) \\
\alpha, \beta, \lambda=\text { constants }
\end{gathered}
$$

Although derived statistically from irradiation test data, this equation was found to contain two basic mechanisms thought to be controlling fuel swelling at burnup levels less than $\sim 1$ metal at. $\%$ :

1) The first mechanism is the accommodation of solid fission products resulting from fission of $\mathrm{U}^{235}$, and is represented by the $\alpha \mathrm{B}$ term in the model.

2) The second mechanism, represented by the $\beta \exp (-\lambda / \mathrm{T})$ term, is attributed to a saturable cavity nucleation phenomenon. This term first appeared as a non-zero intercept, when irradiation data were plotted against burnup.

The NAA $120-4$ is the first experiment in which an apparent saturation of fuel growth has been seen to occur in the first 1000 to $2000 \mathrm{hr}$ of irradiation. This saturation of growth, termed "offset" swelling, was deduced from the rapid decrease in fuel-to-cladding $\Delta T$, experienced during the early part of the irradiation (see Figure 5). Calculations of fuel growth, based on the fuel-to-cladding $\Delta \mathrm{T}$ as a function of time, are shown in Figure 11. These curves indicate that 
CONFDEMTLL RESTRKTTE DATA (GP-1)

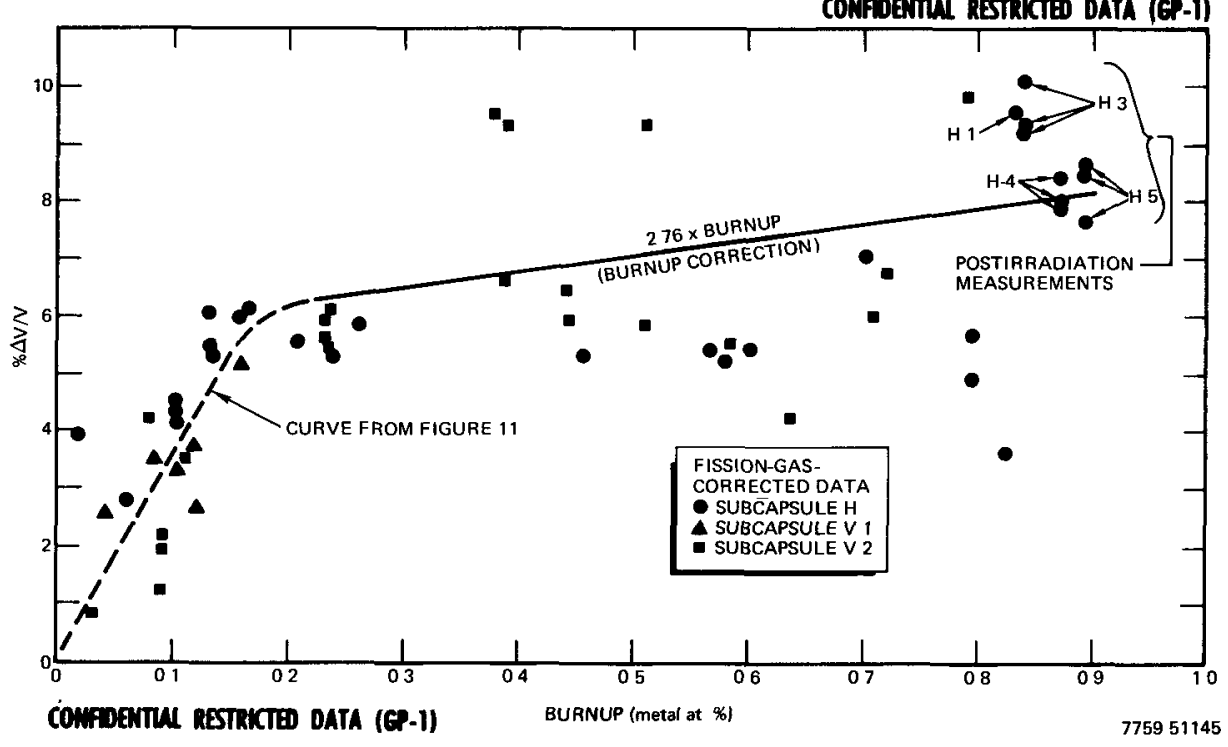

Figure 13. Volume Increase vs Burnup for the $1500^{\circ} \mathrm{F}$ Subcapsules (Based on fuel-cladding $\Delta T$ )

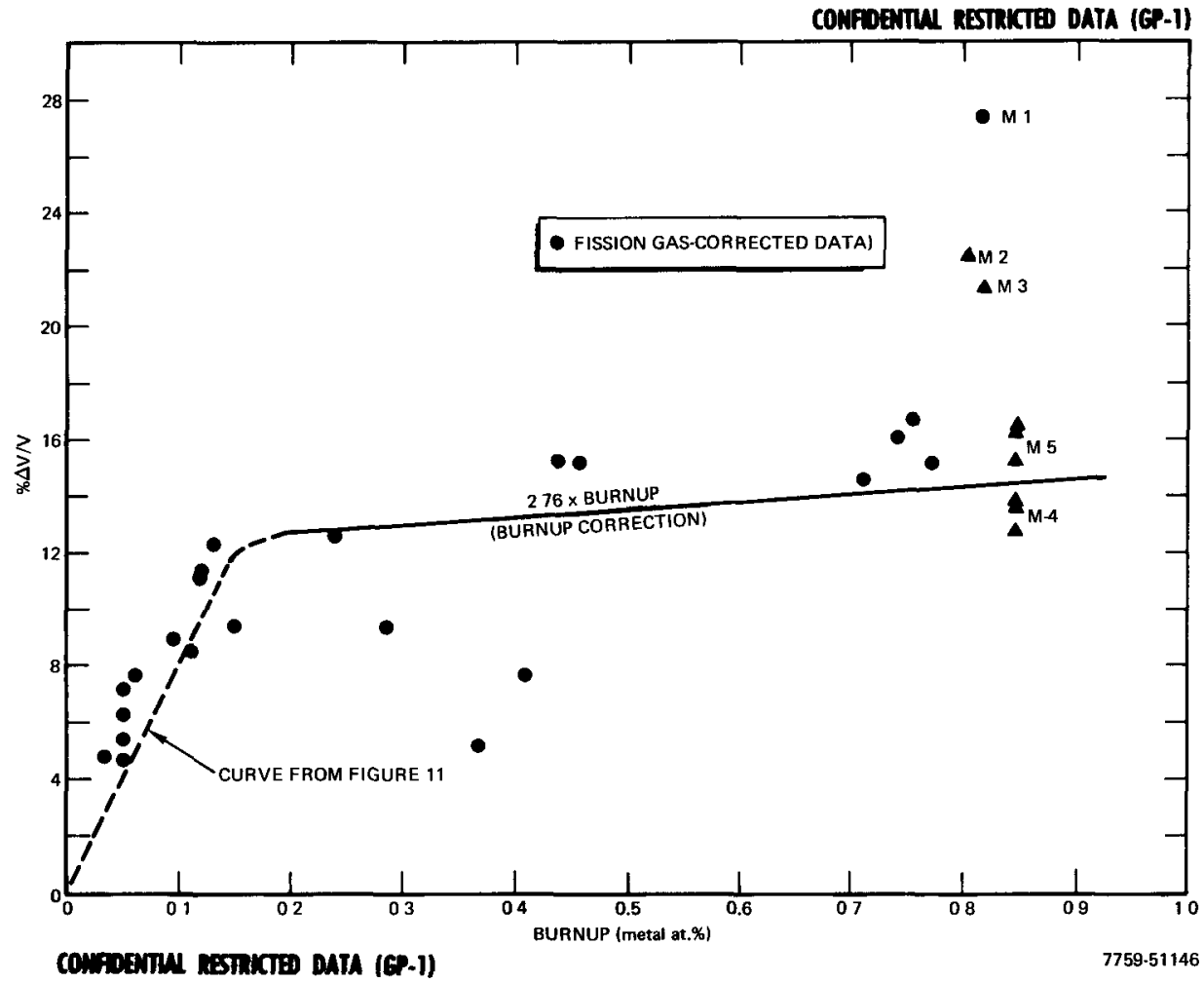

Figure 14. Volume Increase vs Burnup for the $1600^{\circ} \mathrm{F}$ Subcapsules (Based on fuel-cladäing $\Delta \mathrm{T}$ ) 


\section{$\therefore$}

saturation occurs at $\sim 1500 \mathrm{hr}$, for all three experimental temperature levels. These calculations were terminated at the $2100-\mathrm{hr}$ point, due to indicated fission gas release on at least two of the instrumented specimens. The released fission gas is thought to have come from the 20 wt $\%$ end pieces, which operated at $\sim 80^{\circ} \mathrm{F}$ above the center sleeve temperature, and probably entered $\beta$ phase early in life. Further calculations were performed ${ }^{(24)}$ at points in time in which data had been taken at two or more different temperature levels. These calculations involved the simultaneous solution of the two or more sets of data for fission gas concentration in the fuel-to-cladding gap and for gap width. The values of swelling computed by this method are shown in Figures 12, 13, and 14, along with the corresponding curves from Figure 11. The values are shown as $\% \Delta \mathrm{V} / \mathrm{V}$, so that they can be compared directly with final measured volume changes; and are converted from diameter calculations, assuming $\% \frac{\Delta V}{V}=3\left(\% \frac{\Delta D}{D}\right)$. The aforementioned calculations were hampered somewhat by the fact that, due to the test reactor's control rod position, there was a 5 to $10 \%$ flux gradient from the top to the bottom of the fuel specimens. This flux gradient resulted in cladding thermocouples reading 40 to $55^{\circ} \mathrm{F}$ higher on top than at the bottom of the cladding. It will be noted, in Figures 12, 13, and 14, that the spread in the calculated values seems to increase as burnup increases. The reason for the scatter is not known, but is thought to be due to any one or a combination of items, such as instrumentation error, poor assumptions in thermal model, and/or poorly known thermo-physical properties of irradiated materials.

In the present fuel swelling model, (23) once "offset" fuel swelling has been completed, the solid fission product accommodation term becomes dominant. This term is defined as a constant $x$ burnup, and is relatively temperature insensitive. The value of the constant, which appears to be theoretically defensible, has been determined to be 2.76 . This term has been added to the calculated off set swelling, resulting in the curves shown in Figures 12, 13, and 14.

Although these curves do not appear to match the instrumented specimens in any of the subcapsules, they do appear to correlate with some of the uninstrumented specimens. In the case of Specimen $\mathrm{M}-1\left(1600^{\circ} \mathrm{F}\right.$ instrumented specimen), it is known that it, as well as M-2 an $M-3$, enter the $\beta$-phase domain late in the irradiation, because of excessive loss of hydrogen. As has been seen in the past, excessive fuel swelling occurs when an irradiated fuel specimen enters 


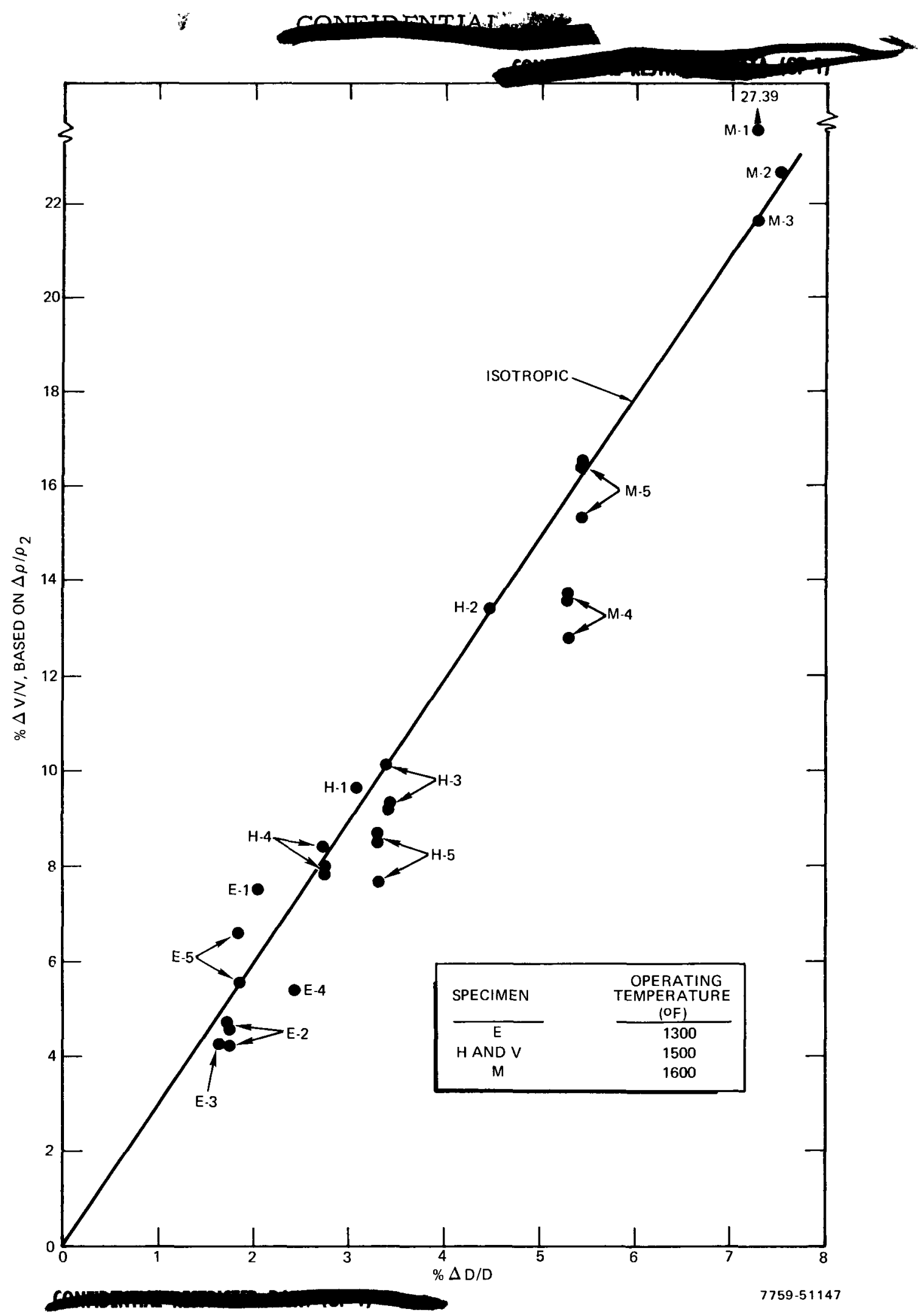

Figure 15. Fuel Volume Changes vs Fuel Diameter Changes (Corrected for hydrogen loss) 


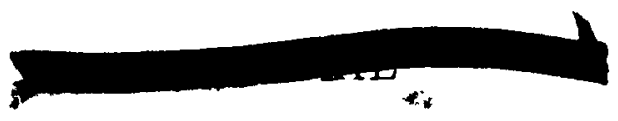

$\beta$ phase. This has been attributed to the $\beta$-phase material's lower strength and/or phase boundary sweeping of fission gases. Specimens M-4 and M-5, which did not enter $\beta$ phase, appear to correlate well with the model.

Specimen H-l also appears to be somewhat higher than the analysis would indicate, but not by a very wide margin. This, again, is probably due to the instrumentation or calculational errors described previously. It is interesting to note the consistency of Specimens $\mathrm{H}-1$ and $\mathrm{H}-3$ (pre-irradiation $\mathrm{H} / \mathrm{Zr}=1.70$ ), and of Specimens $\mathrm{H}-4$ and $\mathrm{H}-5$ (pre-irradiation $\mathrm{H} / \mathrm{Zr}=1.85$ ). According to the phase diagram, these specimens should all have been in 8 phase while at operating temperature. The difference may have been that, during the helium mode of operation, Specimens H-4 and H-5 probably crossed the $8-\epsilon$ phase boundary. There is, however, some uncertainty in the location of this $8-\epsilon$ phase boundary for irradiated $\mathrm{U}-\mathrm{ZrH}$, and it is therefore impossible to state that $\mathrm{E}-4$ and $E-5$ did not cross this phase boundary. Specimens E-1, E-4, and E-5 did swell significantly more than E-2 and E-3, apparently due to the presence of $\epsilon$-phase material.

A plot of diameter changes versus the volume changes based on density measurements is shown in Figure 15. The diameters of some of the specimens were measured at more than one location. The plot shows that most of the data tend to follow the isotropic swelling line. The density of M-l was probably biased by its broken and cracked condition.

In correlating $\mathrm{U}-\mathrm{ZrH}$ fuel swelling data from different irradiation experiments, the greatest success has been achieved by using the "offset, "or equilibrium, bulk average fuel temperature. (23) In the case of a constant cladding temperature experiment, the equilibrium fuel temperature is that temperature which the fuel reaches after offset swelling has been completed. The NAA 120-4 was essentially a constant-fuel-temperature experiment; therefore, the equilibrium temperature was derived differently. Since the operating data indicated that $\sim 1500 \mathrm{hr}$ is needed to reach equilibrium, the highest temperature at which the instrumented fuel specimens operated for $1500 \mathrm{hr}$ or more was determined. The temperatures of the uninstrumented specimens were then obtained, using the temperature of the instrumented specimen and correcting for relative heatgeneration rate (burnup rate), using the mass-spectrographic burnup analyses. 
TABLE 15

SUMMARY OF NAA 120-4 EXPERIMENT DATA

\begin{tabular}{|c|c|c|c|c|c|c|c|c|}
\hline \multirow{3}{*}{$\begin{array}{c}\text { Specimen } \\
\text { Number }\end{array}$} & \multicolumn{2}{|c|}{ Fuel Temperature $\left({ }^{\circ} \mathrm{F}\right)$} & \multirow{2}{*}{\multicolumn{2}{|c|}{$\begin{array}{l}\text { Beginning- } \\
\text { of-Life }\end{array}$}} & \multirow{2}{*}{\multicolumn{2}{|c|}{ End-of-Life }} & \multirow{3}{*}{$\begin{array}{c}\text { Mass- } \\
\text { Spectrographic } \\
\text { Burnup } \\
\text { (metal at. } \% \text { ) }\end{array}$} & \multirow{3}{*}{$\begin{array}{c}\text { Average } \\
\% \frac{\Delta V}{V} \\
\text { (hydrogen } \\
\text { corrected) }\end{array}$} \\
\hline & \multirow{2}{*}{ Design } & \multirow{2}{*}{$\begin{array}{c}\text { Calculated } \\
\text { Equilibrium } \\
\text { Bulk } \\
\text { Average }\end{array}$} & & & & & & \\
\hline & & & $\mathrm{H} / \mathrm{Zr}$ & Phase ${ }^{*}$ & $\mathrm{H} / \mathrm{Zr}$ & Phase $^{\dagger}$ & & \\
\hline$E-1$ & 1300 & 1293 & 1.85 & $\epsilon$ & 1.74 & $\epsilon(\delta+\epsilon)$ & 0.832 & 7.44 \\
\hline$E-4$ & 1300 & 1336 & 1.86 & $\epsilon$ & 1.81 & $\epsilon(\delta+\epsilon)$ & 0.859 & 5.38 \\
\hline$E-5$ & 1300 & 1317 & 1.86 & $\epsilon$ & 1.85 & $\epsilon(\delta+\epsilon)$ & 0.847 & 6.66 \\
\hline$E-2$ & 1300 & 1268 & 1.72 & 0 & 1.73 & $\delta+\epsilon$ & 0.816 & 4.69 \\
\hline$E-3$ & 1300 & 1293 & 1.72 & 0 & 1.71 & $\delta+\epsilon$ & 0.832 & 4.22 \\
\hline $\mathrm{H}-\mathrm{l}$ & 1500 & 1476 & 1.71 & 6 & 1.66 & $\delta+\epsilon$ & 0.832 & 9.57 \\
\hline $\mathrm{H}-3$ & 1500 & 1489 & 1.70 & 0 & 1.70 & $\delta+\epsilon$ & 0.839 & 10.06 \\
\hline $\mathrm{H}-2$ & 1500 & 1518 & 1.71 & 8 & 1.59 & $8+\gamma^{\prime}$ & 0.855 & 13.25 \\
\hline $\mathrm{H}-4$ & 1500 & 1551 & 1.85 & 0 & 1.74 & $\delta+\epsilon$ & 0.874 & 8.40 \\
\hline $\mathrm{H}-5$ & 1500 & 1585 & 1.85 & 8 & 1.79 & $6+\epsilon$ & 0.893 & 8.52 \\
\hline$M-1$ & 1600 & 1560 & 1.71 & $\delta$ & 1.49 & $\delta+y^{\prime}$ & 0.816 & 27.76 \\
\hline$M-2$ & 1600 & 1539 & 1.72 & 8 & 1.44 & $\delta+\gamma^{\prime}$ & 0.805 & 23.32 \\
\hline$M-3$ & 1600 & 1568 & 1.70 & 8 & 1.26 & $\delta+\gamma^{\prime}$ & 0.820 & 25.60 \\
\hline$M-4$ & 1600 & 1620 & 1.85 & 8 & 1.69 & $8+\epsilon$ & 0.847 & 13.68 \\
\hline$M-5$ & 1600 & 1620 & 1.84 & 8 & 1.71 & $\delta+\epsilon$ & 0.847 & 16.30 \\
\hline $\mathrm{V}-1$ & 1500 & 1485 & 1.71 & 0 & 1.49 & $\delta+\gamma^{\prime}$ & 0.816 & 6.65 \\
\hline $\mathrm{V}-2$ & 1500 & 1491 & 1.71 & 8 & 1.62 & $8^{\S}$ & 0.851 & 16.96 \\
\hline
\end{tabular}

*From phase diagram at operating temperature

†From postirradiation metallog raphy (room temperature)

$\S$ Metallographic interpretation indicates $V-2$ entered $\beta$ phase 


\section{-}

These temperatures were then corrected for fuel $\Delta T$, to obtain bulk average fuel temperatures. These temperatures, along with the more pertinent experiment data, are summarized in Table 15. The specimens have been separated according to temperature and $\mathrm{H} / \mathrm{Zr}$, to show the definite influence of hydrogen content on fuel performance.

A plot of reciprocal absolute temperature vs burnup-corrected $\Delta V / V$ shows that the 6 -phase specimens in the experiment correlate fairly well (see Figure 16). The figure also shows the $\epsilon$-phase, as well as $\beta$-phase, material swelled more than the $\delta$-phase material. 


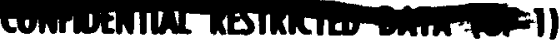

EQUILIBRIUM TEMPERATURE $\left[10^{4} / T\left({ }^{\circ} R\right)\right]$

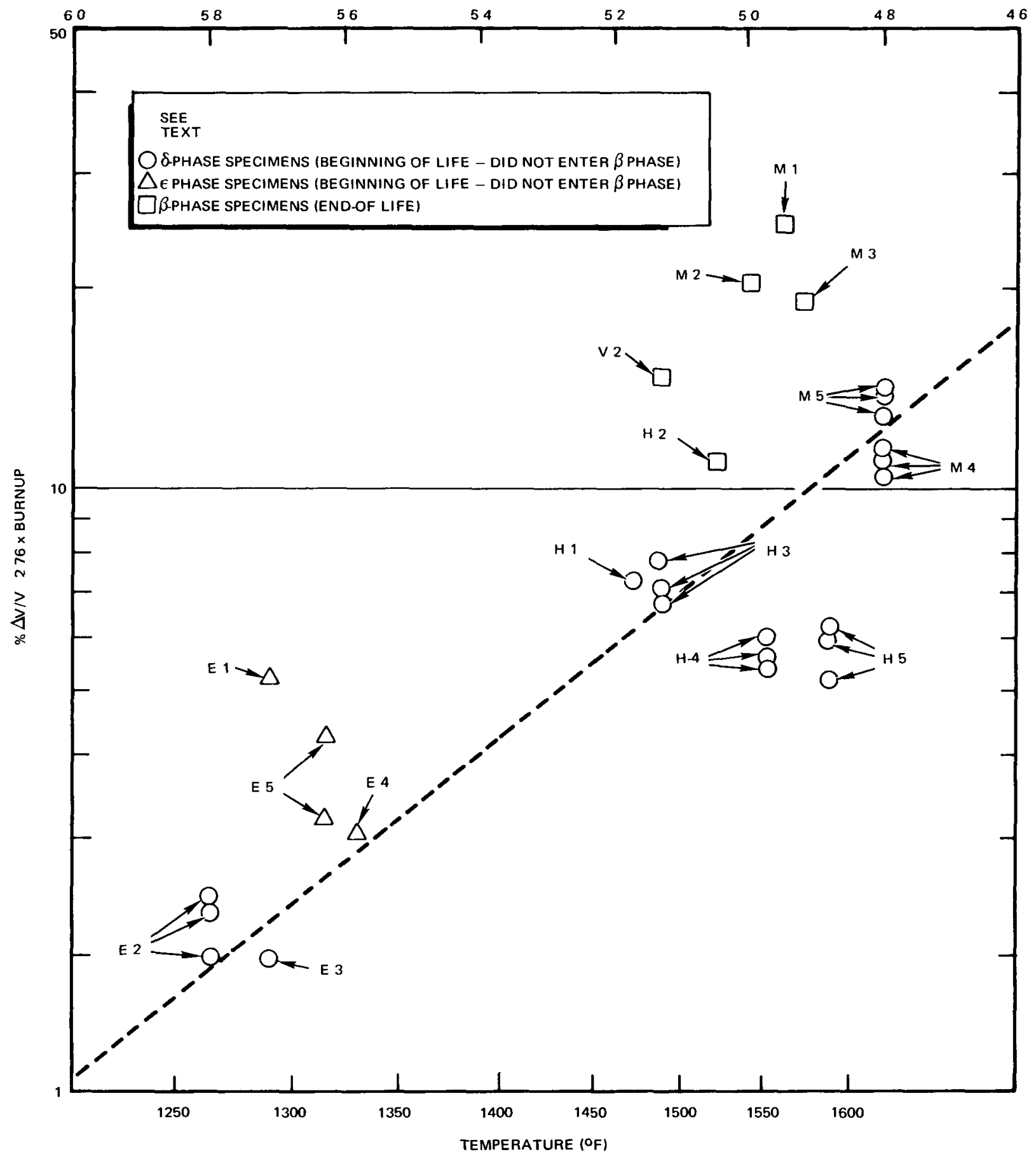

775951148

Figure 16. Burnup-Corrected Volume Change vs Reciprocal Calculated Fuel Temperature 


\section{CONCLUSIONS}

All five subcapsules from the NAA $120-4$ appeared in excellent condition after irradiation. Postirradiation examination showed that all cladding tubes were intact following the test. Only one specimen suffered NaK in-leakage during the test (Specimen V-1), which was attributed to a faulty weld. Burnup levels achieved were within $10 \%$ of the target 0.9 metal at. $\%$.

The NAA 120-4 confirmed the existence of the postulated "offset" fuel growth which saturates early in life. Since the NAA 120-4 was designed to operate at a much higher burnup rate than typical SNAP reactor fuel, the time to saturation growth for SNAP fuel is uncertain. The temperature dependence on time to saturation was not clearly defined by this experiment.

As expected, fuel specimens which entered the $\beta$-phase domain showed volume changes larger than those specimens which operated in the $\delta$-phase domain. Specimens which operated in the $\epsilon$-phase domain also showed larger volume growth than comparable specimens in the 8 -phase domain, although these speciments could have been affected by the $\delta^{-\epsilon}$ phase boundary.

The test results are generally consistent with earlier irradiation data, although exhibiting a somewhat higher temperature dependence than indicated in previous correlations. The data are being correlated with previous irradiation data, under a different program. 
1. C. L. Huffine, "Evaluation of Clad Hydrided Zirconium as Solid Moderator," APEX-335 (November 29, 1956)

2. A. H. Willis and A. J. Moses, "Fission Gas Release From Lightly Irradiated SNAP 8 Fuels, " NAA-SR-10515 (September 15, 1965)

3. P. S. Olson, R. C. Courtright, and R. E. Forrester, "In-Pile Behavior of the SNAP 8 Experimental Reactor Type Sublength Fuel Elements (NAA 115 1 Experiment)," NAA-SR-11996 (November 25, 1966)

4. J. R. Miller and D. A. Polak, "Effects of Irradiation on Hydrided Zirconium-Uranium Alloy, NAA 53 Experiments," NAA-SR-9633 (December 30, 1964)

5. R. C. Courtright and R. E. Forrester, "In-Pile Behavior of the SNAP 8 Experimental Reactor Type Sublength Fuel Elements (NAA 117-1 Experiment), "NAA-SR-12032 (November 30, 1967)

6. R. E. Forrester and W. J. Roberts, "In-Pile Behavior of SNAP 8 Experimental Reactor Type Sublength Fuel Elements (NAA 115-2 Experiment)," NAA -SR-12625 (June 15, 1968)

7. J. S. Buck, W. E. Krupp, and T. A. Park, "Irradiation Performance of a Full-Scale SNAP 2 Reactor Fuel Element (NAA 82-1 Irradiation Experiment)," NAA-SR-12030 (August 15, 1967)

8. J. R. Miller, "Design and Operation of the Phase Change Experiments for the NAA 120-4 Irradiation Experiment, "NAA-SR-MEMO-12260 (December 19, 1966)

9. P. R. Prevo and M. H. Montgomery, "Thermal Neutron Flux Profiles 2A KW, 2B KE, Test Facilities," RL-REA-1056 (April 1965)

10. F. C. Franklin, "Nuclear Analysis for the NAA 120-4 Irradiation Experiment," NAA-SR-MEMO 12459 (June 1967)

11. H. C. Honeck, "A Thermalization Transport Theory Code for Reactor Lattice Calculations," BNL-5826 (September 1961)

12. L. L. Lowe, "NAA 120-4 Heat Transfer and Stress Analysis," NAA-SRMEMO 12392 (April 1967)

13. T. A. Park, "Digital Computer Techniques for the Heat Transfer Analysis of Irradiation Assemblies, "International Symposium on Developments in Irradiation Capsule Technology, Pleasanton, Calif., May 1966

14. R. R. Ligouri and J. W. Stephenson, "The Heating Program (Heat Engineering and Transfer in Nine Geometrics), "ASTRA 417-5-0 (January 1961) 
15. L. M. Maki, "Progress Report, SNAP Reactor Improvement Program, January-March 1966," NAA-SR-11882 (May 23, 1966)

16. J. L. Nummelin and W. H. Graham, "NAA 120-4 Fuel Specimen Assembly Pre-Irradiation Data Package, "NAA-SR-MEMO 12571 (September 1, 1967)

17. D. C. Campbell et al., "SNAP 8 Experimental Reactor Fabrication and Engineering," NAA-SR-8430 (March 1964)

18. L. M. Maki, "Progress Report, SNAP Reactor Improvement Program, October-December 1966," NAA-SR-12292 (February 1967)

19. D. B. West and E. L. Babbe, "NAA 120 Irradiation Experiment Instrumentation," NAA-SR-TDR 12398 (April 1967)

20. J. S. Buck and D. R. Hogle, "NAA 120-4 Operations Guide," NAA-SRMEMO 12455 (May 1967)

21. N. F. Davies, "NAA 120-4 Operations Report," AI-AEC-MEMO 12789 (February 1969)

22. R. E. Forrester, "Post-Irradiation Examination of the NAA 120-4 Irradiation Experiment," AI-AEC-MEMO 12883 (December 1969)

23. R. A. Johnson, "S8DR Evaluation Interim Report, " AI-AEC-MEMO 12901 (November 1969)

24. M. E. Nathan, "Computed Fuel Swelling of the NAA 120-4 Instrumented Fuel Specimens as a Function of Burnup," AI-AEC-TDR 12866 (June 1969) 